\title{
VALIDATION OF THE BODYMEDIA MINI ARMBAND TO ESTIMATE ENERGY EXPENDITURE OF FEMALE BASKETBALL PLAYERS DURING VARIABLE INTENSITY GAME-LIKE CONDITIONS
}

by

\author{
Monica Taylor
}

BS, University of Maine, 2005

MS, East Stroudsburg University, 2008

PhD, University of Pittsburgh, 2012

\author{
Submitted to the Graduate Faculty of \\ School of Education in partial fulfillment \\ of the requirements for the degree of \\ Doctor of Philosophy.
}

University of Pittsburgh 


\title{
UNIVERSITY OF PITTSBURGH
}

\author{
School of Education
}

This dissertation was presented

by

\section{Monica Taylor}

It was defended on

July 10, 2012

and approved by

Fredric Goss, Ph.D., Associate Professor, Health and Physical Activity

Paul Coen, Ph.D., Assistant Professor, Health and Physical Activity

Elaine Rubinstein, Ph.D., Associate Professor, Measurement and Evaluation in Teaching

John Abt, Ph.D., Assistant Professor, Sports Medicine and Nutrition

Dissertation Advisor: Elizabeth Nagle, Ph.D., Assistant Professor, Health and Physical

Activity 
Copyright (C) by Monica Taylor

2012 


\title{
VALIDATION OF THE BODYMEDIA MINI ARMBAND TO ESTIMATE ENERGY EXPENDITURE OF FEMALE BASKETBALL PLAYERS DURING VARIABLE INTENSITY GAME-LIKE CONDITIONS
}

\author{
Monica Taylor, $\mathrm{PhD}$ \\ University of Pittsburgh, 2012
}

Monitoring an athlete's energy intake and energy expenditure (EE) is an important consideration of nutritional planning for sport conditioning and peak performance. In order to provide appropriate recommendations regarding nutritional requirements and caloric needs, an accurate determination of energy requirements is necessary. By knowing an individual's EE, a coach or trainer may be effectively able to determine training loads and volumes necessary for periodization, and seasonal planning for a particular sport. Purpose: To examine the accuracy of the BodyMedia mini armband, to assess EE in female basketball players during various-intensity game-like conditions. Methods: A cross-sectional correlation design with multiple observations was employed. This investigation required three testing sessions, an orientation session, and 2 experimental trial sections. Trials included a maximal multistage 20-m shuttle run (Trial I) and 30-minute basketball skills session (Trial II). The independent variable for this investigation was EE estimated by the Mini armband. The dependent variable was EE determined by the indirect calorimetry (IC) method. Results: EE assessed with the Mini and EE measured with the IC method was significantly correlated for both Trial I $(r=0.839)$ and Trial II $(r=0.833)$. EE calculated by the Mini was significantly underestimated in both Trial I (9.41 \pm 26.1 total kcals) and Trial II (56.71 \pm 14.1 total kcals). During Trial I the underestimation of EE increased with an increase in test level and intensity. Conclusion: Due to the underestimation of EE by the Mini, the development of exercise specific algorithms to improve the estimation of EE during intermittent exercise in basketball players is warranted. 
TABLE OF CONTENTS

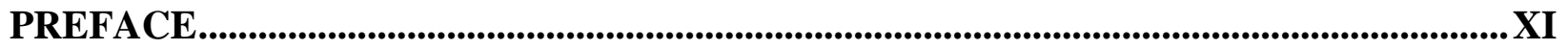

1.0 INTRODUCTION............................................................................................. 1

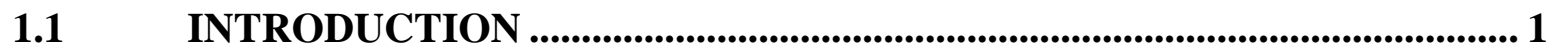

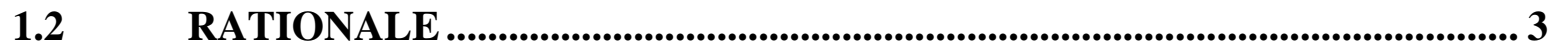

1.3 SPECIFIC AIMS …......................................................................................... 5

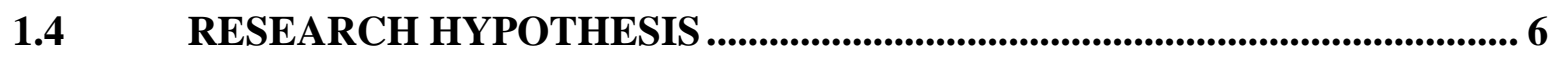

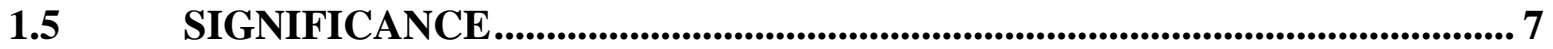

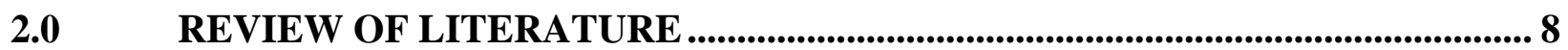

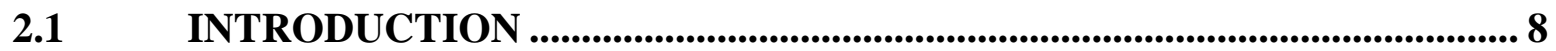

2.2 CRITERION METHODS OF ASSESSING ENERGY EXPENDITURE ... 10

2.2.1 Doubly-Labeled Water .......................................................................................... 10

2.2.2 Direct Calorimetry ................................................................................................ 12

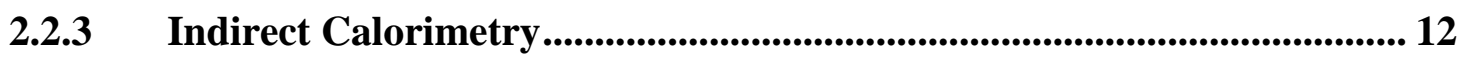

2.2.3.1 Open-circuit indirect calorimeter systems............................................ 13

2.2.3.2 Confinement system (respiratory chambers) ...................................... 16

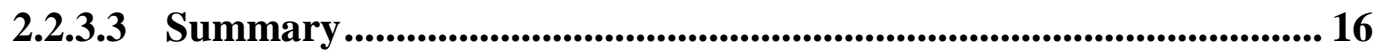

2.3 PORTABLE SYSTEMS TO MEASURE EE....................................................... 17

2.3.1 Heart Rate Methods.................................................................................... 17 
2.3.2 Accelerometers ................................................................................................. 20

2.3.3 HR and Accelerometry (Combined).......................................................... 22

2.3.4 SenseWear Fit Armband .............................................................................. 23

2.3.5 Research for Basketball.............................................................................. 28

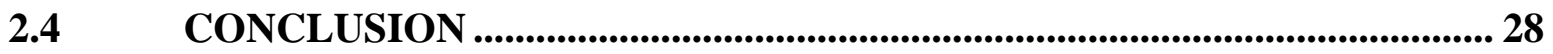

3.0 METHODS ….......................................................................................................... 30

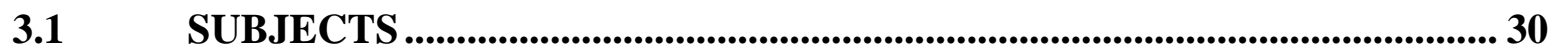

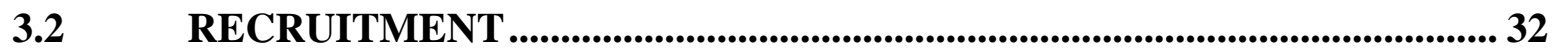

EXPERIMENTAL DESIGN .............................................................................. 32

EXPERIMENTAL PROCEDURES ..................................................................... 33

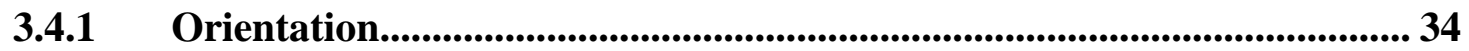

3.4.2 20-m Shuttle Run ............................................................................................ 35

3.4.3 30-Minute Basketball Skills Session................................................................ 36

EXPERIMENTAL TRIAL I AND II.......................................................... 37

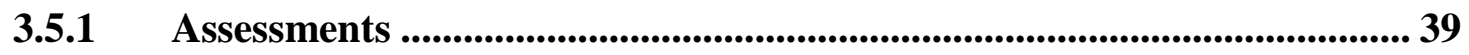

3.5.1.1 Mini Armband to Assess EE .................................................................... 39

3.5.1.2 Indirect Calorimetry to Measure EE ................................................... 39

3.5.1.3 Heart Rate.................................................................................................... 40

3.5.1.4 Rating of Perceived Exertion ....................................................................... 40

STATISTICAL ANALYSIS .................................................................................. 41

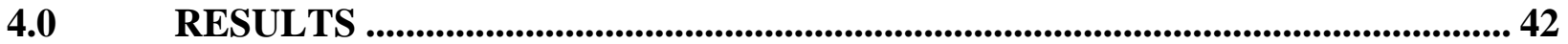

INTRODUCTION ............................................................................................ 42

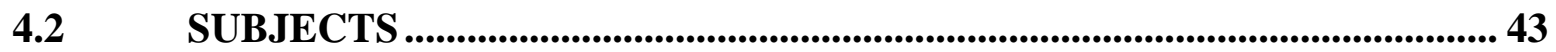




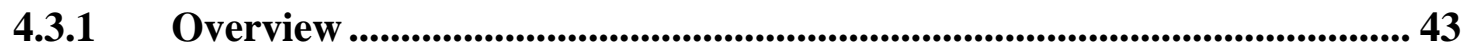

4.3.2 Descriptive Statistics ..................................................................... 44

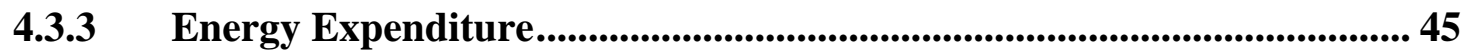

4.3.3.1 Correlations .................................................................................. 46

4.3.3.2 Bland-Altman ................................................................................ 47

4.3.4 Energy Expenditure Estimates Throughout 20-Meter Shuttle Run Test 48

4.4 30 MINUTE BASKETBALL SKILLS SESSION 49

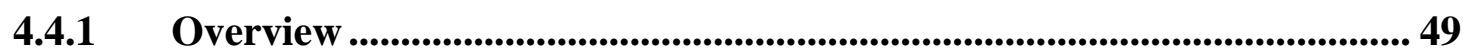

4.4.2 Descriptive Statistics ........................................................................ 50

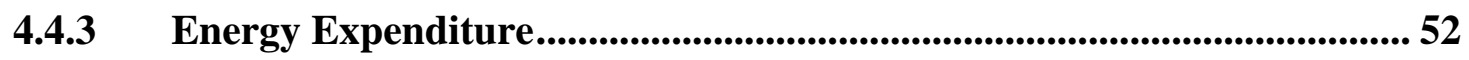

4.4.3.1 Correlations ............................................................................................ 53

4.4.3.2 Bland-Altman ............................................................................... 54

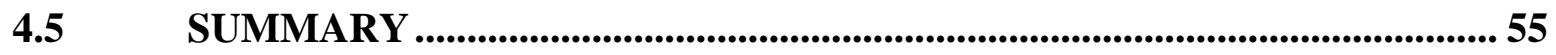

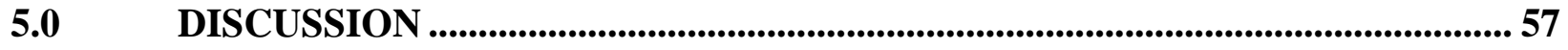

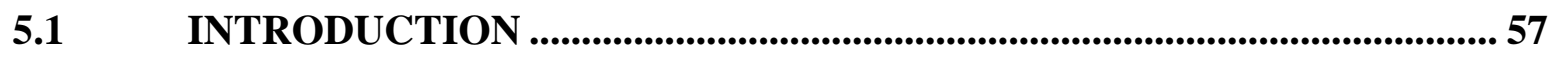

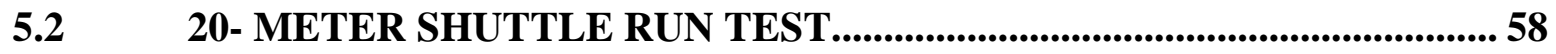

5.2.1 Energy Expenditure.............................................................................. 58

5.2.2 Minute-By-Minute Energy Expenditure............................................ 59

30-MINUTE BASKETBALL SKILLS SESSION ...................................60

5.3.1 Energy Expenditure............................................................................6. 61

5.3.2 Ratings of Perceived Exertion................................................................... 62 
$\begin{array}{llllllll}5.4 & \text { POTENTIAL } & \text { FACTORS } & \text { CONTRIBUTING } & \text { TO } & \text { MINI } & \text { VS } & \text { IC }\end{array}$

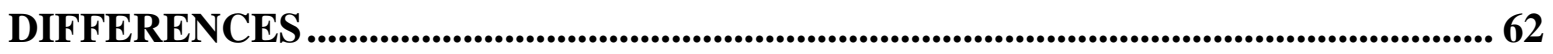

5.4.1 Generalized Algorithms................................................................................... 63

5.4.2 Body Heat Sensor Input ...................................................................................... 64

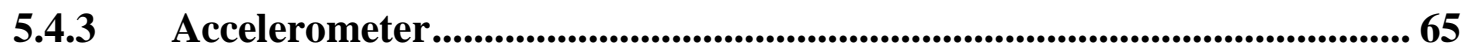

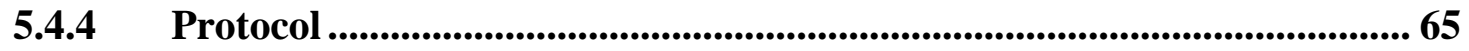

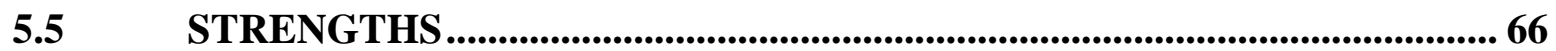

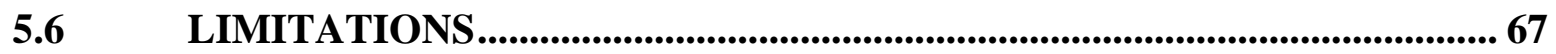

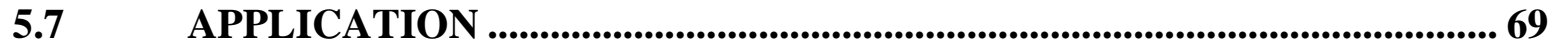

$5.8 \quad$ RECOMMENDATIONS FOR FUTURE RESEARCH .................................. 70

CONCLUSION ..................................................................................................... 71

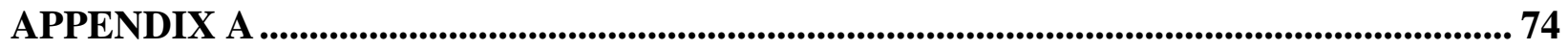

APPENDIX B ..................................................................................................................... 75

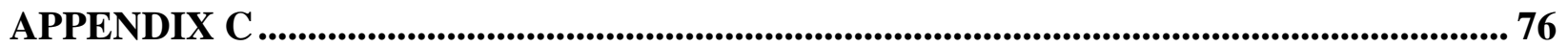

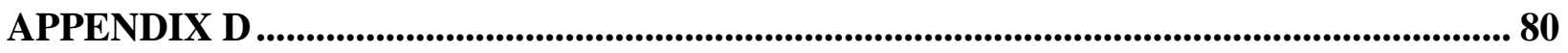

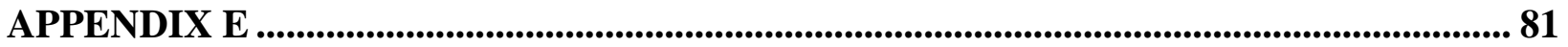

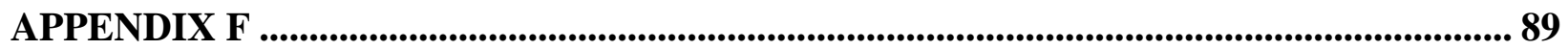

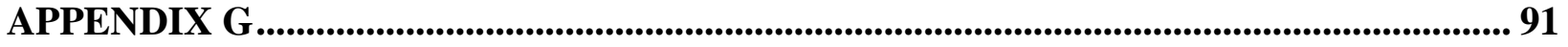

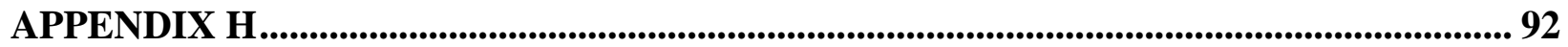

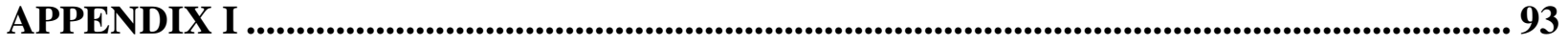

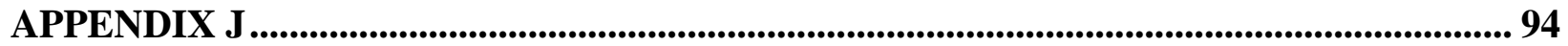

APPENDIX K........................................................................................................................................ 103

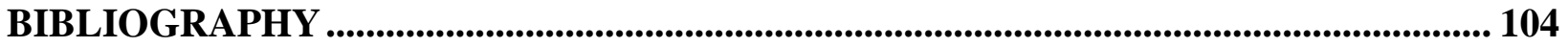




\section{LIST OF TABLES}

Table 1 Open-circuit indirect calorimeter systems ....................................................... 13

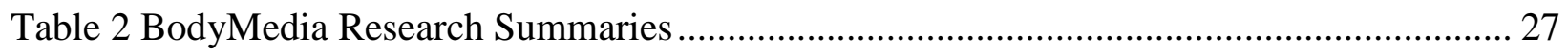

Table 3 Descriptive Characteristics of Subjects .............................................................. 31

Table 4: 30-Minute Basketball Skills Session Protocol......................................................... 37

Table 5 Descriptive Characteristics 20-Meter Shuttle Run Test ............................................ 44

Table 6 Descriptive Characteristics of 30-Minute Basketball Skills Session............................ 51

Table 7 RPE of 30-Minute Basketball Skills Session........................................................... 52

Table 8 Comparison of METs and $\mathrm{kcal} \cdot \mathrm{min}^{-1}$ for 30-Minute Basketball Skills Session.............. 53 


\section{LIST OF FIGURES}

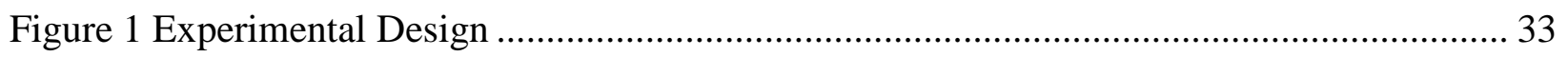

Figure 2 Comparison of EE for 20-Meter Shuttle Run Test .................................................. 45

Figure 3 Association Between Mini and IC From 20-Meter Shuttle Run Test ......................... 47

Figure 4 Bland Altman Plots of Agreement Between IC and Mini for 20-Meter Shuttle Run Test

Figure 5 Mean EE Estimates of Mini and IC Compared Throughout 20-Meter Shuttle Run Test

Figure 6 Comparison of EE (kcals) for 30-Minute Basketball Skills Session............................ 53

Figure 7 Comparison of Total EE (kcals) for 30-Minute Basketball Skills Session ................... 54

Figure 8 Bland Altman Plots of Agreement Between IC and Mini For The 30-minute Basketball

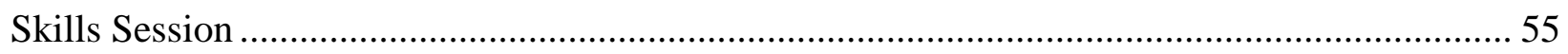




\title{
PREFACE
}

Dr. John Abt

Dr. Elaine Rubinstein

Dr. Fredric Goss

Dr. Paul Coen

Dr. Betsy Nagle

Dr. Ann Hays

Dr. John Jakicic

Thank you for your support and guidance through the dissertation process.

\author{
Jason Taylor \\ Elizabeth Jamison \\ Jamison Family \\ Ward Family \\ Peterson Family \\ Taylor Family
}

Thank you for your patience, support, and guidance and most of all the sacrifices made over the last 3 years. 


\subsection{INTRODUCTION}

\subsection{INTRODUCTION}

Monitoring an athlete's energy intake and energy expenditure (EE) is an important consideration of nutritional planning for sport conditioning and peak performance. In order to provide appropriate recommendations regarding nutritional requirements and caloric needs, an accurate determination of energy requirements is necessary. By knowing an individuals’ EE, a coach or trainer is effectively able to determine training loads, volumes necessary for periodization, and seasonal planning for a particular sport. When caloric intake is not appropriate, changes in body composition may negatively impact overall health and athletic performance (49).

Total energy expenditure (TEE) is the amount of energy needed to meet daily physiological demands (48). Monitored across a 24 hour period, TEE includes the following components: 1) basal metabolic rate (BMR), (i.e. the amount of daily energy expended at rest in order to maintain bodily functions) (48); 2) thermic effect of food (i.e. the amount of energy expended during the digestion, absorption, and transportation of nutrients) (48); and 3) physical activity energy expenditure (PAEE) (i.e. the amount of energy required for any bodily movement produced by skeletal muscle) (48).

PAEE includes an individual's physical activity level (PAL), considered the amount of time a body is in motion throughout a 24-hour period (48). As expected, an athlete's PAL 
reflects a greater EE compared to that of a non-athlete (49). PAL is expressed as the ratio of TEE over BMR. The PAL of an average weight individual (male $70 \mathrm{~kg}$, female $57 \mathrm{~kg}$ ) with a moderately active lifestyle lies between 1.6 and 1.8 (49). In contrast, PAL measures in athletes are considered 2.0 and higher (49), with evidence of up to $70 \%$ of total daily EE occurring through exercise (49). Since the EE of an athlete is considered significantly greater than a sedentary individual, a population specific assessment of EE may be necessary when determining an athlete’s energy intake and daily requirements.

For practical purposes, methods of EE assessment ought to be convenient, reliable, and accurate (45). Presently, EE assessment tools include: 1) accelerometers; 2) pedometers; 3) portable metabolic systems; 4) indirect calorimetry (IC); and 5) doubly-labeled water (DLW). Despite the potential advantages of each technique, limitations associated with a lack of validity, reliability, or practicality has been shown in studies using free-living environments $(67,87,88$, $18,19,38,73,14,84,32,75,26,36,50,13)$. To date, few studies have examined the accuracy of assessing EE using athletic populations in sports specific environments. This demonstrates a need to identify accurate methodology that can assess EE for athletes while performing sports specific tasks.

The SenseWear Mini Armband (Mini) (BodyMedia ${ }^{\circledR}$, Inc., Pittsburgh, PA), a multi sensor device worn on the upper arm, provides measures of EE during periods of physical activity, and has been examined in adults, children, and clinical patients (31, 22, 39, 40, 78, 43, 51, 52). However, few investigations have explored the validity of this instrument using intensities similar to a specific athletic event, or in free-living environments. This includes athletes who engage in intermittent play at varying intensities such as basketball players. Therefore, the proposed investigation examined the validity of the Mini during variable intensity 
game-like conditions using a sample of women’s basketball players.

\subsection{RATIONALE}

TEE is the amount of energy needed to meet daily physiological demands (48). BMR represents $60-70 \%$ of TEE in most individuals (48), with the remaining $30-40 \%$ from the thermic effect of food and physical activity level. TEE measured as a unit of heat may be expressed as total kilocalories ( $\mathrm{kcal})$, calories per min $\left(\mathrm{kcal} \cdot \mathrm{min}^{-1}\right)$ or relative to an individual's body weight $\left(\mathrm{kcal} \cdot \mathrm{kg} \cdot \mathrm{min}^{-1}\right)$. Although considered gold standard measures of EE (70), doubly labeled water (DLW) and indirect calorimetry (IC) techniques are not without limitations (67, 86, 18, 38, 73, 75, 12, 54). For example, IC cannot easily assess free-living subjects, while DLW does not provide information on the pattern or intensity of physical activity $(67,86,18,38,73,75,12$, 54). These methods are considered costly and require trained technicians for test administration and interpretation (67, 86, 18, 38, 73, 75, 12, 54). Recently, additional methods to assess physical activity have been developed for free-living environments $(47,89,91)$. These devices include physiological data monitors that measure heart rate (HR) and body temperature, and motion sensor devices (pedometers and accelerometers) $(47,89,91)$. Considered an accurate estimate of EE, HR monitors rely on the underlying assumption of a linear relationship between HR and oxygen consumption $(57,37,21,25,16,26)$. However, these relationships have not been observed during low or high intensity activity, therefore, HR monitors may provide a less accurate estimation during particular exercise intensities (57, 37, 21, 25, 16, 26). HR may also be affected by factors independent of whole body movement (i.e. environmental and psychological stressors, caffeine, and certain medications), the result of which can produce increases in HR 
without a significant increase in oxygen consumption $(25,37,26)$.

Accelerometry is a method of predicting EE where the ability to detect body motion and accelerations produced by a body segment/limb can be measured through movement in space (65). Unlike other methods, accelerometers are capable of accurately detecting and predicting energy cost of physical activity under conditions of both low and high intensity. However, the accuracy of accelerometry is highly dependent upon the type of activity performed (65, 44, 82, 59). Accelerometers have been shown to inaccurately predict energy cost of particular activities including cycling, swimming, rowing, upper-body exercise, stair-climbing, lifting, carrying a heavy load, and walking/running on a graded surface (65, 44, 82, 59). Therefore, for many athletes, this limits the utility of accelerometers for estimating EE in freeliving conditions.

To increase the accuracy of predicting EE, the BodyMedia ${ }^{\circledR}$ Fit Armband Mini (Mini) utilizes a combination of physiological and mechanical measurement systems. Worn on the upper arm, this device collects data through a variety of sensors that include: accelerometry, galvanic skin response, near-body ambient temperature, skin temperature, and heat flux (3). Participant data may be uploaded and analyzed for a minute-by-minute breakdown of energy requirements for all physical activities performed (3).

Previous investigations involving the Mini have focused primarily on adult populations. Although considered accurate, the Mini may overestimate or underestimate the energy cost for certain physical activities (22, 31, 39, 40, 43, 78). The accuracy of the armband is also reliant on population or activity specific algorithms (3). However, concerns may exist regarding the appropriateness of these algorithms when used for particular populations. Few investigations have assessed the validity of the Mini armband for high intensity exercise in 
endurance-trained individuals $(43,22)$. Koehler, et al. found the armband to underestimate TEE in endurance athletes (43). Drenowatz and Eisenmann found EE measured by the armband to be inaccurate around intensities of 10 metabolic equivalents (METs) suggesting a possible "ceiling effect” (22). The Mini has yet to be investigated in an athletic performance environment, and for athletes not predominately endurance trained. Therefore, the purpose of this investigation was to validate the Mini as a measure of EE during variable intensity basketball game-like conditions.

\subsection{SPECIFIC AIMS}

\section{The primary aim of this investigation was to:}

1. Validate the accuracy of the BodyMedia ${ }^{\circledR}$ FIT Armband Mini in measuring energy expenditure (EE) of female basketball players during a 30-minute variable intensity basketball skill and game-like condition.

\section{The secondary aim of this investigation was to:}

2. Validate the accuracy of the BodyMedia ${ }^{\circledR}$ FIT Armband Mini in measuring energy expenditure (EE) of female basketball players during a 20 meter shuttle running test of aerobic capacity. 


\subsection{RESEARCH HYPOTHESIS}

\section{Primary Hypothesis}

1. It was hypothesized that EE (kcal per session, METs, and $\mathrm{kcal} \cdot \mathrm{min}^{-1}$ ) measured by the Mini during variable intensity basketball skill and game-like conditions would show no significant difference when compared to EE (kcal per session, METs, and $\mathrm{kcal} \cdot \mathrm{min}^{-1}$ ) as measured by indirect calorimetry (IC).

2. It was hypothesized that EE (kcal per session, METs, and $\mathrm{kcal} \cdot \mathrm{min}^{-1}$ ) measured by the Mini $\left(\mathrm{kcal} \cdot \mathrm{min}^{-1}\right)$ during variable intensity basketball skill and game-like conditions would demonstrate significant positive correlations to EE (kcal per session, METs, and $\mathrm{kcal} \cdot \mathrm{min}^{-1}$ ) as measured by indirect calorimetry (IC).

\section{Secondary Hypothesis}

3. It was hypothesized that EE (kcal per session, METs, and $\mathrm{kcal} \cdot \mathrm{min}^{-1}$ ) measured by the Mini during a 20 meter shuttle running test of aerobic capacity would show no significant difference when compared to EE (kcal per session, METs, and $\mathrm{kcal} \cdot \mathrm{min}^{-1}$ ) as measured by indirect calorimetry (IC).

4. It was hypothesized that EE (kcal per session, METs, and $\mathrm{kcal} \cdot \mathrm{min}^{-1}$ ) requirements measured by the Mini during a 20 meter shuttle running test of aerobic capacity would demonstrate significant positive correlations to oxygen consumption (kcal per session, METs, and $\mathrm{kcal} \cdot \mathrm{min}^{-1}$ ) as measured by indirect calorimetry (IC). 


\subsection{SIGNIFICANCE}

Results of this investigation explained the ability of the Mini armband to accurately estimate EE in an athletic population. Furthermore, it was anticipated that the proposed study would provide athletes, coaches, and trainers with an accurate measure of the caloric demands of collegiate female basketball players during simulated game-like conditions. Athletes have unique energy demands for peak performance that require sufficient caloric intake, adequate hydration, and appropriate timing of meals. This information may assist with the determination of caloric needs to properly maintain body composition throughout a competitive season. In addition, results of this study may help quantify the energy demands associated with anaerobic and aerobic training drills and sets. This can provide insight to coaches when considering metabolic demands of specific workout components, and methods to improve workout designs and assessments of recovery needs. 


\subsection{REVIEW OF LITERATURE}

\section{$2.1 \quad$ INTRODUCTION}

Total energy expenditure (TEE), the amount of energy needed to meet daily physiological demands, is a direct function of all physiological and metabolic processes necessary for the exchange of energy (46). The first law of thermodynamics states that energy is neither created nor destroyed; rather it is transformed from one form to another (17). Chemical energy from food (carbohydrates, proteins, fat, and alcohol) is liberated in the presence of $\mathrm{O}_{2}$ and transformed to a form of mechanical energy, such as muscular contraction. Considered a byproduct of this transformation, heat released may be measured by calorimetry. Measurements of heat (energy) may be reflected as kilojoules (KJ) or kilocalories (kcal) (17). A kilojoule (KJ) can be defined as the energy used when 1 kilogram $(\mathrm{kg})$ is moved 1 meter $(\mathrm{m})$ by the force of 1 newton $(\mathrm{N})(49)$. A kilocalorie ( $4.184 \mathrm{KJ})$ can be defined as the energy needed to raise the temperature of 1 gram of water from 14.5 to 15.5 degrees Celsius (49). TEE may be expressed as total kcal, kcal per min $\left(\mathrm{kcal} \cdot \mathrm{min}^{-1}\right)$ or relative to an individual's body weight $\left(\mathrm{kcal} \cdot \mathrm{kg} \cdot \mathrm{min}^{-1}\right)$.

As separate components of TEE, 1) basal metabolic rate (BMR); 2) thermic effect of food; and 3) physical activity energy expenditure (PAEE) are influenced through the interaction of nutritional, genetic, and environmental factors (89). BMR covers the minimum energetic costs of the processes essential for life (48). It is the energy required to sustain the human body's vital 
functions and typically measured in a wakeful, rested, and post-absorptive state. The thermic effect of food is the increased heat production from the digestion, absorption, and conversion of food (61). PAEE is the EE associated with muscular contractions and maintaining body posture (61). Under most circumstances, the BMR of an individual accounts for the largest proportion of TEE and is primarily determined by fat-free body mass, gender, thyroid hormones, and protein turnover (56). For individuals with a sedentary lifestyle this can account for $60 \%$ of the total daily EE (61). The thermic effect of food is approximately $10 \%$ of the total daily EE in those consuming an average mixed diet. Activity-induced EE is the most variable component of TEE, with its contribution ranging from $10-30 \%$ based on activity level (61).

Quantifying activity-induced EE accurately can be challenging, particularly in free-living environments. The criterion measures for assessing EE of physical activity in a laboratory setting include indirect calorimetry (IC) and doubly labeled water (DLW). Due to cost and required expertise, recent alternative techniques have included the following measures: 1) self-report (physical activity diaries, interviews, surveys, and questionnaires); 2) physiological data (HR); and 3) motion sensor devices (pedometers, accelerometers). The validity and reliability of these techniques that measure EE in free-living environments will be explored in the following section. Specifically, review of the literature will demonstrate a need for a portable device that will allow for a valid estimate of EE in a free-living athletic environment. 


\subsection{CRITERION METHODS OF ASSESSING ENERGY EXPENDITURE}

\subsubsection{Doubly-Labeled Water}

The DLW method assesses TEE under laboratory and field conditions and is based on the rate of metabolic $\mathrm{CO}_{2}$ production $\left(\mathrm{VCO}_{2}\right)$, where $\mathrm{O}_{2}$ consumption is estimated from $\mathrm{CO}_{2}$ production (67). DLW consists of the stable water isotopes hydrogen $\left({ }^{2} \mathrm{H}\right.$ on deuterium) and oxygen $\left({ }^{18} \mathrm{O}\right)$, and is administered to subjects as a liquid, which is dosed according to body size. Labeled hydrogen leaves the body as water in sweat, urine, and pulmonary water vapor $\left(2 \mathrm{H}_{2} \mathrm{O}\right)$, and labeled oxygen leaves as water $\left(\mathrm{H}_{2} \mathrm{O}\right)$ and carbon dioxide $\left(\mathrm{C}^{18} \mathrm{O}_{2}\right)(67)$. Urinary excretions of these isotopes are tracked with mass spectrometry over several days, and oxygen uptake $\left(\mathrm{VO}_{2}\right)$ and $\mathrm{EE}$ are extrapolated from $\mathrm{VCO}_{2}$ using established equations (67). The turnover rate of body water is greater than ${ }^{2} \mathrm{H}$ because ${ }^{18} \mathrm{O}$, not $\mathrm{H}$, is lost via respiratory $\mathrm{CO}_{2}$ (67). The DLW method is based on the following underlying assumptions: 1) isotopes label only body water and carbon dioxide; 2) ${ }^{2} \mathrm{H}$ is lost as water; 3) ${ }^{18} \mathrm{O}$ is lost as water and $\mathrm{CO}_{2}$; 4 ) water and $\mathrm{CO}_{2}$ output rates are constant; 5) isotope losses are not fractionated; and 6) isotope intake rates are constant. The DLW technique has been validated against IC and is considered a "gold standard" for determining EE in free-living conditions $(67,85,18,38,73)$.

Schoeller and Webb compared the DLW method to a respiratory gas exchange (RGE) procedure in subjects throughout five days in a laboratory living environment where cycle ergometer or treadmill workload bouts were observed several times a day. A non-significant result suggested the EE compared between DLW and RGE differed by only 6\% using measured RQ (68). A follow-up study by Schoeller et al., examined differences in isotope dose when DLW was compared to RGE (69). Subjects were housed in a respiratory chamber for 4 days, received 
${ }^{2} \mathrm{H}_{2}{ }^{18} \mathrm{O}$ at either a low $(n=6)$ or a moderate $(n=3)$ isotope dose, and exercised at a given workload 3 times each day. When compared to RGE, there was an overestimation of $\mathrm{CO}_{2}$ production for both the low, and moderate dose isotope groups.

Seale et al., examined the accuracy of DLW compared to a room-sized respiratory calorimeter using nine subjects observed over 5-7 days. When compared to the DLW technique, there was no significant difference in EE (73). Westerterp et al., compared the DLW method to calorimetry in 2 groups each having a different activity level (low and high). DLW was in agreement between both groups, with the low group being $1.4 \pm 3.9 \%$ higher, and high activity group being on average 1.0\% lower than calorimetry (87). In a later study by Forbes-Ewan et al., DLW was found to be within 5\% agreement (kcal/day) when calculating EE in soldiers training for jungle warfare (29). This suggests the DLW technique shows strong agreement to indirect calorimetry when measuring EE in a variety of settings. However, despite the accuracy and precision of DLW, the technique has several limitations. DLW requires expensive isotopes, with a given dose ranging from $\$ 800-\$ 1500$ per subject $(67,85,18,38,73)$. Although DLW provides an accurate representation of daily EE in free-living environments, it does not provide information regarding patterns of physical activity. Therefore, it cannot differentiate the duration, frequency, or intensity of particular forms physical activity $(67,85,18,38,73)$. Specifically, since it is necessary to collect urine for a period of 7-14 days, DLW can only provide data relative to average daily TEE, rather than acute bouts of physical activity. This limits the use of this technique when determining how patterns of activity or acute bouts of activity contribute to TEE and health-related outcomes. 


\subsubsection{Direct Calorimetry}

Direct Calorimetry (DC) assesses the metabolic rate of humans via the measurement of heat production, similar to the method for determining energy value of food using a bomb calorimeter (56). A human calorimeter directly measures the body's EE (heat production) by detecting changes in water temperature circulated through a series of coils at the top of a chamber (56). This method is rarely used due to the length of subject confinement, cost, and trained personnel needed to conduct testing (56).

\subsubsection{Indirect Calorimetry}

Indirect calorimetry (IC), the measurement of energy produced when nutrients are oxidized (74), it is determined by measuring $\mathrm{O}_{2}$ consumption and $\mathrm{CO}_{2}$ production rather than directly measured heat production (74). There are four methods utilized to measure indirect calorimetry: 1) Open circuit; 2) Ventilated open circuit; 3) Expiratory collection open circuit; and 4) Confinement system. Primary characteristics of these methods are identified in Table 1.

IC estimates EE from $\mathrm{O}_{2}$ consumption and $\mathrm{CO}_{2}$ production using open and closed circuit spirometry. The open circuit IC technique measures heat production through respiratory gas exchange by analyzing oxygen consumption $\left(\mathrm{VO}_{2}\right)$ and carbon dioxide $\left(\mathrm{CO}_{2}\right)$ production by the body (28). In contrast, when using a closed-circuit system, a subject inhales oxygen instead of room air as used in open-circuit systems. In a closed-circuit system, expired air will return to containers filtered through a soda line (Bicarbonate), which absorbs carbon dioxide. Changes in volume of oxygen in the system are recorded as the volume of oxygen consumed $\left(\mathrm{VO}_{2}\right)$ (28). Open-circuit IC is commonly used as a criterion method when assessing EE in a laboratory 
setting, and considered an accurate and valid measure of short-term EE (28). Open-circuit systems (expiratory collection) methodology will be included in the present investigation. The following sections will expand in further detail.

Table 1 Open-circuit indirect calorimeter systems

\begin{tabular}{|c|c|c|c|c|c|c|}
\hline & $\begin{array}{c}\text { Mouth } \\
\text { Piece, } \\
\text { Mask, } \\
\text { or } \\
\text { Hood }\end{array}$ & $\begin{array}{c}\text { Room Air } \\
\text { Pumped } \\
\text { Through } \\
\text { System }\end{array}$ & $\begin{array}{c}\text { Laboratory } \\
\text { Use Only }\end{array}$ & $\begin{array}{c}\text { Inspired and } \\
\text { Expired } \\
\text { Gases } \\
\text { Measured }\end{array}$ & $\begin{array}{c}\text { Confined } \\
\text { Room/Chamber }\end{array}$ & $\begin{array}{c}\text { Only Expired } \\
\mathrm{O}_{2} \text { Measured }\end{array}$ \\
\hline $\begin{array}{c}\text { Ventilated } \\
\text { Open Circuit }\end{array}$ & 0 & 0 & 0 & 0 & & \\
\hline $\begin{array}{c}\text { Expiratory } \\
\text { Collection } \\
\text { Open Circuit }\end{array}$ & 0 & 0 & & & & \\
\hline $\begin{array}{c}\text { Confinement } \\
\text { System }\end{array}$ & & & 0 & 0 & & \\
\hline
\end{tabular}

$(11,68,52)$

\subsubsection{Open-circuit indirect calorimeter systems}

Using an open-circuit indirect calorimeter system, inspired and expired gases are analyzed (12). Metabolic measurements obtained from the determination of inspired and expired gases include: 1) determination of oxygen consumption $\left.\left(\mathrm{VO}_{2}\right) ; 2\right)$ carbon dioxide production $\left(\mathrm{VCO}_{2}\right)$; and 3) respiratory quotient (RQ). Open-circuit systems measure EE over several hours or days depending on the experimental design. There are considered two types of open-circuit systems: 1) ventilated open-circuit; and 2) expiratory collection systems (12). 


\subsection{Ventilated open-circuit systems}

Ventilated open-circuit indirect calorimeter systems involve components that: 1) collect mixed expired air; 2) measure flow rate; 3) analyze gas concentrations; and 4) pump air through a system (75). The open-circuit indirect calorimeter has both ends of a measuring system open to atmospheric pressure. Inspired and expired air are separated by a three way respiratory valve or non-breathing mask. The expired gases are then collected within a Douglas bag or similar chamber for analysis of $\mathrm{O}_{2}$ and $\mathrm{CO}_{2}$ content (75).

Using a mouthpiece, mask, or transparent hood/canopy, expired air is drawn out of the collection device using a pump. The expired air is mixed using a fan and/or mixing chamber, and the sample of expired air is dried and analyzed for $\mathrm{O}_{2}$ and/or $\mathrm{CO}_{2}$ concentrations (12). Oxygen is generally analyzed through paramagnetic analyzers, while carbon dioxide is analyzed by infrared analyzers (12). Alternatively, a mass spectrometer may be used to measure the gas concentrations (12). Ventilated open-circuit indirect calorimeters have precision within 0.5-2\% $(74,12)$. For a ventilated hood or canopy, response time may range from 30 seconds to 2 minutes $(74,12)$.

\subsection{Expiratory collection open-circuit systems}

Similar to ventilated systems, expiratory collection open-circuit systems involve components that: 1) collect mixed expired air; 2) measure flow rate; 3) analyze gas concentrations; and 4) pump air through a system. The expiratory collection open-circuit systems include smaller portable systems compared to the larger ventilated systems, and are not limited to laboratory use 
only. The expiratory system also differs from the ventilated system because it only measures expired $\mathrm{O}_{2}$ and not expired $\mathrm{CO}_{2}$ (54). Development of small portable indirect calorimeters (e.g., Cosmed $\mathrm{K} 4 \mathrm{~b} 2$ ) has allowed field assessments of $\mathrm{O}_{2}$ uptake, from which EE can be estimated (54). These devices include a mouthpiece or a mask attached to a one-way valve where expired air may enter. The flow rate of expired air through the valve is measured, and a small proportion of expired air is diverted to a gas storage reservoir analyzed at the end of each measurement period $(75,12)$. Measures may be obtained intermittently for up to 2 days. Recently, various modifications have allowed air to be drawn through the system at a fixed rate $(75,12)$. A validation study performed by McLaughlin et al., compared the $\mathrm{K} 4 \mathrm{~b}^{2}$ against the criterion Douglas bag technique (DB) during rest and stationary cycling in 10 subjects (54). The stationary cycling was performed at 5 different power outputs (50, 100, 150, 200, $250 \mathrm{~W})$ and there were no significant differences found between the Cosmed and DB for rest and power outputs up to $150 \mathrm{~W}$ (54). Another study by Duffield and colleagues compared the Cosmed to indirect calorimetry during a treadmill test of varying speeds in 12 physically fit males on four separate occasions (23). Results of this study revealed the Cosmed to have satisfactory test-retest reliability (23).

Expiratory collection open-circuit systems can be considered both uncomfortable and costly. This may limit the usefulness of this method for quantifying EE in settings where multiple units are needed such as athletic practices or games $(75,12,54)$. Recent technological advances have resulted in the design of more precise, robust, and dependable portable calorimeters. Furthermore, the unique advantage of a portable device will allow EE to be measured in free-living environments. 


\subsubsection{Confinement system (respiratory chambers)}

Using a respiratory chamber, a subject is placed inside a room/chamber of known volumes in which there are sophisticated sensing devices to quantify physical activity. In this setting, measurements can be performed for up to several days (75). Response time for a room or chamber system can vary from 5 to 30 minutes depending on the software, air mixing, and room volume. $\mathrm{O}_{2}$ consumption and $\mathrm{CO}_{2}$ production are estimated from changes in concentrations of these gases in chamber air over time (75). When using a respiratory chamber, a subject is placed inside a tightly sealed container of a known volume. These chambers usually have a bed, television, toilet, TV, Internet, and all other basic comforts. Subjects are typically locked in the chamber for 24 hours or more (75). A period of observation may be prolonged by periodically flushing the chamber with fresh air. Confinement systems have shown errors of $2 \%$, and response times of about 50 minutes (75). Currently, confinement systems are rarely used due to the increased use of the DLW method, along with advancements in portable measurement systems and other laboratory techniques. Confinement systems are also expensive and require significant laboratory space (75). The time required for participants to stay in a chamber can also cause challenges for recruitment (75).

\subsubsection{Summary}

Although IC is accurate in determining EE, there are several limitations that impact its ability to assess EE under free-living conditions. In addition, testing is typically restricted to controlled laboratory settings. IC systems that assess EE are costly (approximately $\$ 20,000$ to $\$ 100,000$ per system), and require well-trained personnel. The use of a respiratory metabolic system requires a 
mouthpiece or a facemask to collect breathing samples that may be uncomfortable or fit improperly. Furthermore, errors may occur in measurements due to escaped expired air. Therefore, the utility of a portable system may offer a greater advantage when estimating EE in free-living environments.

\subsection{PORTABLE SYSTEMS TO MEASURE EE}

\subsubsection{Heart Rate Methods}

Heart rate (HR) measurement uses a personal monitoring device and telemetry system, which allows exercise intensity throughout a session to be monitored in real time (16). As a means of predicting EE, HR monitoring relies on the underlying assumption of a linear relationship between $\mathrm{HR}$ and oxygen consumption $\left(\mathrm{VO}_{2}\right)$, and thus, between $\mathrm{HR}$ and $\mathrm{EE}$ (16). This requires the determination of an individual's $\mathrm{VO}_{2} / \mathrm{HR}$ regression line where $\mathrm{EE}$ is predicted (16). Evidence has shown that HR monitors provide accurate assessments of moderate intensity activity between $110-150$ beats per minute $\left(\mathrm{b} \cdot \mathrm{min}^{-1}\right)$. However, HR monitors appear to provide a less accurate assessment of EE during low $\left(<110 \mathrm{~b} \cdot \mathrm{min}^{-1}\right)$, or high $\left(>150 \mathrm{~b} \cdot \mathrm{min}^{-1}\right)$ intensity activities (85, 32, 76, 26, 37, 50, 15). Furthermore, gender, weight, $\mathrm{VO}_{2}$, $\mathrm{BMI}$, high ambient temperature, high humidity, hydration level, posture, illness, emotional stress, caffeinated drinks, and age appears to be factors influencing the relationship between HR and EE. Previous studies have shown that gender, body weight, age, and HR are needed to accurately estimate EE during physical activity $(36,53,26,40)$.

Terbizan et al., examined the validity of seven HR monitors (including two Polar HR 
monitors), through comparison to an electrocardiogram (ECG) measurement system (85). In this

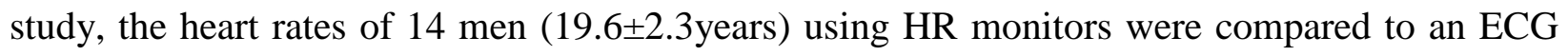
for 10 seconds rest and during treadmill exercise at $85.7 \mathrm{~m} \cdot \mathrm{min}^{-1}$, $107.3 \mathrm{~m} \cdot \mathrm{min}^{-1}$ and 160.8 $\mathrm{m} \cdot \mathrm{min}^{-1}$. All 7 of the HR monitors demonstrate accuracy during rest and moderate exercise $(\mathrm{r} \geq$ 0.90 $\left.\mathrm{SEE} \leq 5 \mathrm{~b} \cdot \mathrm{min}^{-1}\right)$. However, as speeds increased, the accuracy compared to ECG monitors decreased, suggesting further investigation of the accuracy of HR monitors at higher treadmill speeds is needed (85).

Goodie et al., (32) determined that the Polar HR monitor could accurately measure heart rates of participants (18-48 years), during rest and stressful tasks when compared to an ECG system (32). As a concurrent measure, it was found the Polar monitors produced accurate HR values $(r=0.90)$ when compared to the ECG. Although, the Polar HR monitor provided slightly higher absolute HR measurements, the measures were not significantly different (32).

Spurr, et al., performed a comparison of EE between whole body IC and HR recordings. When subjects were housed in a respiratory chamber for 22 hours while performing 6 exercise bouts wearing the HR monitor, there was no significant difference between the two methods (77).

While HR monitors have demonstrated valid measures during rest and moderate exercise, an accurate prediction of EE may be influenced by limitations demonstrated with the HR method. Therefore further investigation is needed to determine its accuracy in measuring EE during vigorous exercise.

\section{FLEX HR Method}

The FLEX HR is an individually predetermined HR used to discriminate between rest and 
physical activity during a monitoring period (89). The FLEX HR is defined as the mean of the highest HR during rest, and the lowest HR during light exercise (89). The FLEX HR method involves the simultaneous monitoring of $\mathrm{HR}$ and $\mathrm{O}_{2}$ consumption for each individual while lying down, sitting, standing, and performing various intensities of physical activity, and is used as a reference point to determine how EE is calculated. If a subject's HR during an experimental trial is below their FLEX HR, resting metabolic rate is used to determine EE. However, if a subject's HR is above the FLEX HR, a subject's individual HR/oxygen consumption calibration curve will be utilized to predict EE (89, 53, 25).

Several studies have examined the accuracy of the FLEX HR method with mixed results $(89,53,25)$. Livingstone and colleagues examined the accuracy of the FLEX HR method as a measure of EE (53). Calibration curves were used to assign an energy value to minute-by-minute recorded HR above the predetermined FLEX HR, and compared to EE values collected by the DLW method for each day. Results indicated that, on average, the HR method over estimated. Ekelund, et al., compared the estimates of TEE obtained by two different methods of FLEX HR with TEE determined by the DLW technique in athletes (25). Results indicated no significant differences between the DLW and two FLEX HR methods. However, a significant difference was shown between the two methods of FLEX HR (25). This suggests further investigation is needed to determine the accuracy of the Flex HR method in estimating EE.

\section{Summary}

HR monitors have low subject burden and are considered convenient devices for assessment in a free-living environment. Although the HR-VO2 relationship is linear across a wide range of physical activity intensities, this is frequently not the case during low and very high intensity activity $(85,32,75,26,37,50,14)$. Because many daily activities include low to 
moderate intensity, HR monitoring may not provide precise estimates of daily EE among freeliving individuals. HR monitors also need individual calibration, and are influenced by factors that include gender, BMI, fitness level, high ambient temperature, high humidity, hydration level, posture and illness, emotional stress, and caffeinated drinks. This makes HR monitoring a less suitable mode of quantifying the relationship between physical activity and EE in healthrelated research.

\subsubsection{Accelerometers}

Accelerometers are activity monitors that continuously measure the intensity, frequency, and duration of movement over time $(15,58)$. Accelerometers are piezoelectric sensors that detect acceleration(s) or movement in one to three orthogonal planes (anteroposterior, mediolateral, and vertical), and convert this acceleration into digital signals used to predict EE (15). This technique is based on the theoretical construct that acceleration is directly proportional to muscular force, and therefore, EE (15). Processed data may be recorded by internal memory and downloaded through computer ports. Classified as uni-axial or tri-axial, these accelerometers may vary in size, price, and capabilities. Typically considered small as well as portable, accelerometers can collect EE in free-living environments (58).

\section{Uniaxial Accelerometers}

Uniaxial accelerometers measure accelerations in one direction, typically the vertical plane (58). Examples include stepping, walking, and running. Several investigators have examined the validity of the uniaxial accelerometer compared to the IC method. Montoye et al., found the accelerometer was less accurate and underestimated the increased energy cost of walking or

running at an incline (58). Swartz et al., examined the accuracy of a Computer Science 
Application (CSA) accelerometer worn on the hip and wrist to estimate EE of activities of daily living (82). Results showed the CSA monitor both underestimated and overestimated the EE of certain daily activities when compared to IC. Regression equations significantly under-predicted the actual measured energy cost of mowing with a power mower and a manual mower, and significantly over-predicted the measured energy cost of ironing, caring for children, and slow walking (82). Similarly, in a study by Leenders et al., the CSA monitor significantly underestimated physical activity EE when compared to DLW during a 7-day free-living time period (46).

Results of previous investigations suggest there are limitations of uniaxial accelerometers for predicting EE across a wide spectrum of physical activities (46, 82, 58, 42). This may be due to the uniaxial accelerometer's ability to detect movement in only single rather than multiple planes. The development of tri-axial accelerometers has recently occurred.

\section{Tri-axial Accelerometers}

Tri-axial accelerometers are three dimensional motion sensors designed to measure accelerations in three planes, typically identified at the waist (16). The Tritrac, RT3, IC Sensors, were developed to assess body acceleration in multiple planes of space with the assumption that recording motion in more than one plane would increase the validity and accuracy of predicting EE (16).

Using a Tri-axial accelerometer, Bouten et al., examined the assessment of EE for physical activity while performing normal daily activities for 3 minutes each. Using the IC method as a criterion standard, results showed all activities for the estimation of EE were similar between the two methods (9). Campbell and colleagues (14) examined the ability of the Ttrac-R3D tri-axial accelerometer to measure $\mathrm{EE}$ when compared to the Cosmed $\mathrm{K} 4 \mathrm{~b}^{2}$ portable IC system. 
Participants completed 5-min bouts of walking, jogging, stair climbing, walking on an incline, stationary cycling, and arm ergometry at self-selected speeds that mimicked activities of daily living. Results showed the Tritrac overestimated the EE (as measured by the $K 4 \mathrm{~b}^{2}$ ) while walking, and jogging, and underestimated EE for stair climbing, stationary cycling, and arm ergometry (14). A study conducted by Rowlands and colleagues examined the ability of the RT3 triaxial accelerometer to assess PA compared to the Tritrac accelerometer and IC. Similar to previous studies, the accelerometer was strongly correlated to IC when measuring overall EE. However, the accelerometer overestimated EE of sedentary activities, and underestimated the EE of high intensity activities (65).

Based on the previous results, there appears to be a relationship between accelerometry and criterion measures of EE during periods of physical activity. However, these devices tend to significantly underestimate absolute EE during static exercises, walking upstairs, carrying any excess loads, and cycling $(82,6,15,58,33,42,9,13)$. Thus, this may limit the utility of these devices to quantify EE during periods of structured and free-living physical activity.

\subsubsection{HR and Accelerometry (Combined)}

The combination of both HR and accelerometry may be advantageous when attempting to estimate EE, and allow researchers to have a more accurate assessment of EE in free-living environments. Strath, et al., compared EE from a combined accelerometer and HR system to a criterion measure of EE (indirect calorimetry) (79). A non-significant difference in EE was reported for this combined system when compared to IC ( $p>0.34)$. When examined separately, the accelerometry underestimated EE by an average of 1.1 METs $(p<0.001)$, whereas HR significantly overestimated EE by an average of 0.4 METs $(p<0.001)$. The HR accelerometer 
technique was more strongly associated with IC $(r=0.81)$, than HR $(r=0.67)$, or accelerometry ( $r=0.54)$ compared individually (79). These data suggest the combination of HR and accelerometry improves the estimation of EE during selected activities compared to either method alone. Similar results were reported in a follow-up study conducted by Strath, et al., when EE measured by IC was compared to the combination system (HR and accelerometry) ( $r=$ 0.81) $(\mathrm{p}<.001)(80)$. Moreover, there was no significant difference between EE measured by IC $\left(749 \pm 138 \mathrm{MET} \cdot \mathrm{min}^{-1}\right)$ vs. the combination system $\left(748 \pm 178 \mathrm{MET} \cdot \mathrm{min}^{-1}\right)(79)$. While the combination of HR and activity monitoring may provide a method to accurately estimate EE, further validation studies are necessary to determine the ability of this technique to estimate EE during various forms of physical activity in free-living environments.

\subsubsection{SenseWear Fit Armband}

The Mini (BodyMedia ${ }^{\circledR}$, Inc., Pittsburgh, PA) is a portable device, which incorporates multiple parameters into an estimate of EE (kcal), and can continuously monitor physiological data such as physical activity, steps per minute, and EE (3). Worn on the back of an individual's right arm, the Mini includes the following four sensors: 1) a two-axis accelerometer that tracks movement and body position; 2) a heat-flux sensor which determines heat dissipated from the body through heat loss measured between the skin and a vent on the side of the armband; 3) sensitive thermistors which measure skin temperature; and 4) a sensor which measures galvanic skin responses (GSR) due to sweating and emotional stimuli (3). In addition to demographic characteristics (age, gender, weight, height, right or left handedness, smoker or non-smoker), data for each of these parameters is collected by the armband and stored in the device for later transfer to a computer. Further analysis incorporates algorithms used to estimate EE (3). The 
implementation of multiple methods of detection may enable the Mini to overcome the limitations observed in other assessment devices. It may also allow for the accurate assessment of EE during non-weight bearing activities such as cycling, stair stepping, resistance exercise, or activities involving only upper body movement, and non-ambulatory physical activity.

\section{Validation Studies Conducted in Adults}

Mealey and colleagues examined the accuracy of the SenseWear Pro Armband (SWA) when EE was measured during simulated common daily activities (57). When subjects participated in 60 minutes of activities designed to simulate daily movements that included multiple repetitions of sitting, standing and walking, no significant differences in EE were found between the SWA and the IC methods (57). Similarly, Fruin and Rankin examined the validity of the SWA to estimate EE during rest, treadmill walking, and cycling (31). During the period of rest, EE predicted by the SWA $\left(1.3 \pm 0.1 \mathrm{kcal} \cdot \mathrm{min}^{-1}\right)$ was not significantly different from the criterion measure of IC $\left(1.3 \pm 0.1 \mathrm{kcal} \cdot \mathrm{min}^{-1}\right)$. During treadmill walking, the SWA was also significantly related to the criterion measure $(r=0.76)(p<0.004)$. During cycling, TEE predicted by the SWA $\left(352.9 \pm 20.3 \mathrm{kcal} \cdot \mathrm{min}^{-1}\right)$ did not differ significantly from the IC method $\left(372.2 \pm 60.4 \mathrm{kcal} \cdot \mathrm{min}^{-1}\right)(\mathrm{p}>0.28)$ although measures were poorly correlated $(\mathrm{r}=0.03-0.12)$. The study also showed that the SWA significantly over-estimated EE by $13-27 \%$ when walking with no grade and significantly under estimated EE by $22 \%$ when walking at a grade of $5 \%$ (p $<$ 0.002). Modest correlation coefficients were reported between EE estimated from the SWA and IC during walking, with correlations ranging from $r=0.47$ to $r=0.69(p<0.04)$. These results suggest that the SWA appears to both over and under estimate walking speeds when compared to the IC method of measuring EE in adults (31). When comparing the validity of the SWA for estimating EE during treadmill walking and running, King, et al., also found the SWA to over- 
estimate EE for both men and women at walking and running speeds (42).

Jakicic and colleagues (39) examined the accuracy of the SWA to estimate EE during four separate modes of activity that included: 1) treadmill walking; 2) stair stepping; 3) cycle ergometry; and 4) arm ergometry. During each exercise protocol, EE was simultaneously measured by the IC criterion measure. Original algorithms developed by the manufacturer revealed intraclass correlations for EE of: 1$)$ treadmill ( $\mathrm{r}=0.77$ ) (CI: 0.57-0.88); 2) stair stepping ( $\mathrm{r}=0.63)(\mathrm{CI}$ : 0.39-0.79); 3) cycling $(\mathrm{r}=0.28)(\mathrm{CI}$ - $-0.05-0.56)$; and 4) arm ergometry $(r=0.74)(C I: 0.55-0.86)$ when compared to the IC method. However, compared to IC the SWA significantly underestimated TEE during walking $(14.0 \pm 17.5$ kcals $)$, cycling $(32.4 \pm 18.8$ kcals), and stair stepping (28.2 \pm 20.3 kcals), while TEE for arm ergometry was significantly overestimated by $21.7 \pm 8.7 \mathrm{kcals}$. When exercise-specific algorithms were applied to the data, intraclass correlations for the SWA generally improved [1) treadmill ( $r=0.87$ ) (CI: -.75-0.93); 2) stair stepping $(\mathrm{r}=0.82)(\mathrm{CI}: 0.58-0.92)$; and 3) cycling $(\mathrm{r}=0.89)$ (CI: 0.74-0.95). When corrected, these exercise-specific algorithms showed no significant differences in TEE between the SWA and indirect calorimetry. These results were considered encouraging after refined algorithms were applied to the data (39).

Using high intensity exercise, Drenowatz and Eisenmann validated the SWA Armband using 20 endurance-trained subjects. Subjects performed 3 separate bouts of 10-minute treadmill running at different intensities (65\%, 75\%, and $85 \%$ ), in addition to a 30 -minute self-paced outdoor run. When the SWA Armband was compared to the IC system, results indicated that the SWA Armband significantly underestimated EE at intensities above 10 MET's, or a running speed of 6 mph (22). Similar to the results of Drenowatz and Eisenmann, Koehler et al., found the SWA to consistently underestimate EE at higher running speeds (43). Fourteen male 
endurance athletes were recruited and monitored for 7 days with 2 controlled exercise trials. When the armband was compared to the DLW method, results showed a positive correlation of $\mathrm{r}=0.73, \mathrm{p}<0.01$ between TEE assessed with the armband vs. the DLW technique. The SWA significantly underestimated EE for most exercise intensities, and the underestimation increased as exercise intensity increased (43).

Few studies have compared the armband to the DLW method of measuring TEE. St. Onge et al., examined the accuracy of the armband when compared to the DLW method in free-living adults (78). Forty-five subjects were asked to wear the armband for a 10-day period while only removing it for showering and other water activities. Results indicated the armband significantly underestimated EE $(-117 \mathrm{kcal} / \mathrm{d}) ;(\mathrm{P}<0.01)$. In addition, daily EE was $2375 \pm 366 \mathrm{kcal} / \mathrm{d}$ compared to $2492 \pm 444 \mathrm{kcal} / \mathrm{d}$ (DLW) over the 10-day period (78). The following table summarizes the validation studies of SWA compared to IC and DLW. (Table 2) 
Table 2 BodyMedia Research Summaries

\begin{tabular}{|c|c|c|c|c|}
\hline Study & $\begin{array}{c}\text { Criterion } \\
\text { Reference }\end{array}$ & Results & $\mathrm{R}$ value & Standard Error \\
\hline $\begin{array}{c}\text { Drenowatz et } \\
\text { al. }\end{array}$ & IC & High intensity UE & $r=0.66$ & - \\
\hline $\begin{array}{c}\text { Fruin and } \\
\text { Rankin }\end{array}$ & IC & $\begin{array}{l}0 \% \text { grade OE } \\
5 \% \text { grade UE }\end{array}$ & $\begin{array}{l}r=0.54 \\
r=0.47\end{array}$ & - \\
\hline Jakicic et al. & IC & $\begin{array}{c}\text { Walk UE } \\
\text { Cycle UE } \\
\text { Stepping UE } \\
\text { Arm Erg OE }\end{array}$ & $\begin{array}{l}r=0.87 \\
r=0.89 \\
r=0.82 \\
r=0.66\end{array}$ & $\begin{array}{l}14.9 \pm 17.5 \mathrm{kcal} \\
32.8 \pm 18.8 \mathrm{kcal} \\
28.2 \pm 20.3 \mathrm{kcal} \\
21.7 \pm 8.1 \mathrm{kcal}\end{array}$ \\
\hline $\begin{array}{c}\text { Johannsen et } \\
\text { al. }\end{array}$ & IC & TEE UE & $\mathrm{r}=0.85$ & - \\
\hline Koehler et al. & DLW & $\begin{array}{l}\text { TEE agreement } \\
\text { Trend towards: } \\
\text { UE high intensity } \\
\text { OE low intensity }\end{array}$ & $\mathrm{r}=0.73$ & $\begin{array}{c}65 \pm 665 \mathrm{kcal} \cdot \mathrm{d} \\
1\end{array}$ \\
\hline St. Onge et al. & DLW & Daily EE UE & $r=0.81$ & - \\
\hline $\begin{array}{l}\text { Wadsworth et } \\
\text { al. }\end{array}$ & DLW & $\begin{array}{c}\text { Walking } \\
\text { Agreement } \\
1^{\text {st }} \text { Rest } \\
\text { Agreement } \\
2^{\text {nd }} \text { Rest } \\
\text { Agreement } \\
\text { TEE Agreement }\end{array}$ & $\begin{array}{l}r=.94 \\
r=.79 \\
r=.83 \\
r=.95\end{array}$ & $\begin{array}{l}- \\
-\end{array}$ \\
\hline
\end{tabular}

TEE- Total Energy expenditure, UE- Under-Estimates, OE- Over-Estimates

Previous studies reveal a lack of consistency when the SWA measures energy expenditure (31, 22, 39, 40, 78, 43, 51, 52), suggesting further exploration of the accuracy in the SWA method at varying exercise intensities is warranted. Furthermore, it is possible that 
additional algorithms modeled for athletes or higher intensity exercise maybe needed to improve the estimation of EE from the SWA technique.

\subsubsection{Research for Basketball}

The majority of basketball-related sports performance research has investigated injury prevention treatment (65). Specifically investigations have focused on the etiology of ACL injuries and preseason, in-season, and post-season training strategies that both cause and prevent these conditions (60). Independent of strength and conditioning research, few investigations have studied the metabolic aspects of basketball skills and training (60). Furthermore, there has been no research exploring energy requirements of basketball throughout a competitive season.

\subsection{CONCLUSION}

Additional investigations that assess EE in free-living environments should include athletic populations. Due to methodological issues and feasibility of testing in these environments, few investigations have used IC and DLW criterion measures to determine the EE of sports-specific patterns in their natural environments. Although some limitations may apply, current portable devices (accelerometers, HR monitors, pedometers, etc.) may provide alternative methods for assessing EE. In particular, the more recent development of the Mini may offer promise as a method for measuring EE in free-living conditions because it could provide a multi sensor approach to calculating EE compared to other methods. Furthermore, the Mini also allows for a more user-friendly method of monitoring and collecting data, alleviating technician error. Few 
studies have explored the validity of the SWA in athletes, with no published reports examining its ability to measure throughout intermittent sports play. Therefore, the primary aim of this study was to examine the validity of the SWA to estimate EE during variable intensity game-like conditions in Division I and III female basketball players. 


\subsection{METHODS}

The primary purpose of this study was to validate the BodyMedia® FIT Mini Armband (Mini) as a measure of energy expenditure (EE) throughout free-living environments. Specifically, the intended study examined variable intensity basketball game-like conditions using female basketball players. All procedures were approved by the Institutional Review Board at the University of Pittsburgh. Written and informed consent was obtained from all participants prior to participation in this study (APPENDIX J). Pilot work for this protocol was done for 4 months leading up to the start of subject recruitment. 12 young healthy males and females age 18-22 were used to solidify the protocol used for experimental trial II along with testing the flow and feasibility of each trial session.

\subsection{SUBJECTS}

Sixteen women's college basketball players (aged 18-23 years) who were currently participating in basketball and conditioning activities participated in this investigation. Specifically eight females from the University of Pittsburgh and eight from Carnegie Mellon University volunteered as subjects in the present study. All participation was strictly voluntary. The study did comply with NCAA regulations, and had the support and approval of the University of Pittsburgh and Carnegie Mellon University Department of Athletics, head women’s basketball 
coaches, and head strength and conditioning coaches. The racial, gender, and ethnic characteristics of the subject population reflected the demographics of female basketball players recruited to participate in NCAA Division I and Division III women's basketball (90). Descriptive characteristics are explained in Table 3. In order to participate, subjects were: 1) healthy; 2) currently eligible for college athletics; and 3) able to complete an orientation and two experimental trials. Exclusion criteria for the study included: 1) responding, "yes” to one or more questions on the physical activity readiness questionnaire (PAR-Q); 2) presence of any orthopedic, musculoskeletal, neurological, cardiac, and/or any medical conditions that prohibit exercise; 3) presence of diabetes, hypothyroidism, or any other medical conditions that would affect energy metabolism; 4) reported use of medications or any performance enhancing drugs that may have affected heart rate, blood pressure, metabolism, and/or EE responses; 5) knowingly pregnant or pregnant within the last 6 months; or 6) unwilling to perform or participate in two basketball experimental trials.

Table 3 Descriptive Characteristics of Subjects

\begin{tabular}{|c|c|c|c|c|c|}
\hline & Pitt (n=8) & CMU (n=8) & Guards (n=10) & Forwards (n=6) & Combined (n=16) \\
\hline Age (yrs) & $18.9 \pm 1.1$ & $19.5 \pm 1.2$ & $19 \pm 1.1$ & $19.5 \pm 1.4$ & $19.2 \pm 1.2$ \\
\hline Weight (kg) & $75.5 \pm 17.2$ & $75.6 \pm 8.1$ & $70.4 \pm 9.9$ & $84.3 \pm 13.5$ & $75.6 \pm 12.9$ \\
\hline Height (cm) & $178.6 \pm 9.1$ & $176.3 \pm 5.6$ & $172.6 \pm 3.2$ & $185.5 \pm 4.4$ & $177.4 \pm 7.4$ \\
\hline BMI (kg· m) & $23.4 \pm 3.5$ & $24.5 \pm 3.4$ & $23.7 \pm 3.7$ & $24.3 \pm 3.1$ & $23.9 \pm 3.4$ \\
\hline Body Fat (\%) & $23.3 \pm 3.5$ & $24.1 \pm 5.9$ & $24.3 \pm 5.5$ & $22.6 \pm 3.4$ & $23.7 \pm 4.7$ \\
\hline
\end{tabular}

Values are presented as Mean \pm Standard Deviation. 


\subsection{RECRUITMENT}

The women's basketball head coaches and strength and conditioning coaches at each university were informed of the study. They were provided with a brief overview of the purpose, clinical significance, anticipated outcomes, and practical application of this investigation. Once University of Pittsburgh IRB approval was obtained, the primary investigator announced the study to team members. All participation was strictly voluntary. Decision to participate in the study had no bearing on eligibility status, nor did it interfere with practice time. Athletes were asked to contact the researcher if interested in participating in the study. If interested, they were

invited to attend an informal one-hour group orientation session where all aspects of the research project were addressed. This group orientation session included an overview of tests conducted, purpose of the study, as well as risks and benefits of the investigation. Potential subjects were encouraged to ask questions at this time regarding all procedures. If they met preliminary screening and inclusion criteria, and agreed to participate, potential subjects were asked to complete the informed consent as approved by the IRB of the University of Pittsburgh. Subjects were informed of their right to withdraw from participation at any time during the investigation.

\subsection{EXPERIMENTAL DESIGN}

A cross-sectional correlational design with multiple observations was employed. This investigation required three testing sessions: 1) Orientation session; 2) Experimental Trial I; and 3) Experimental Trial II. Experimental Trials I and II included: 1) a maximal multistage 20-m 
shuttle run; and 2) a 30-minute individual training session. The two experimental trials were separated by approximately 24-72 hours. The Independent variable for this investigation was energy expenditure (EE) [total kcals, METs, $\mathrm{kcal} \cdot \mathrm{min}^{-1}$ ] estimated by the Mini armband. The dependent variable was EE [total kcals, METs, $\mathrm{kcal} \cdot \mathrm{min}^{-1}$ ] determined by the IC method.

\subsection{EXPERIMENTAL PROCEDURES}

This investigation consisted of an orientation session and two Experimental Trials I and II (See

Figure 1.). The following testing trials are outlined in Figure 1 and sections 3.4.1 to 3.4 .3 to follow.

Orientation

- Overview of study

- Medical History/Par-Q

- Informed Consent

- Test Battery Explanation

- Test Battery Video

\section{Experimental Trial I}

- Anthropometrics

- 20 m shuttle experimental trial

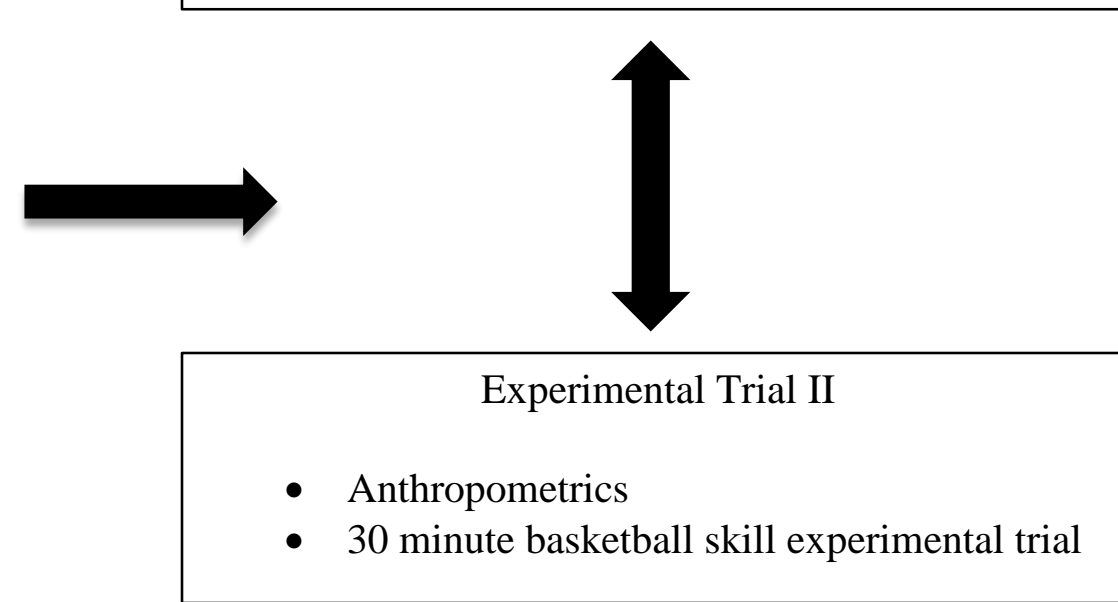

Figure 1 Experimental Design 


\subsubsection{Orientation}

During the one-hour group orientation session, subjects were provided an overview of the study. Subjects completed the PAR-Q (Appendix $\mathrm{H}$ ) as well as a medical history questionnaire (Appendix G) in order to be screened for eligibility to participate. If all subjects were not able to attend the group orientation session due to classes or other conflicts, a second session was offered for those unable to attend the first session. If the medical history questionnaire indicated any contraindications to exercise testing or subjects answered "yes" to any of the PAR-Q questions they were excluded from the study. Potential risks and benefits, along with the study rationale were explained. Informed Consent was obtained during the orientation session.

Verbal and visual explanations of Experimental Trials I and II were provided. An explanation was given for all drills related to the 30-minute basketball workout session. Subjects were shown a diagram, along with a short video clip of each drill executed throughout the 30minute basketball workout. Subjects also listened to a one-minute segment of the 20-m shuttle run recording to become familiar with the sounds of the recording. In addition, subjects were oriented to the OMNI Walk/Run perceived exertion scale (RPE) using standardized instruction and anchoring procedures (Appendix F). Subjects were able to observe and wear all equipment in order to become oriented to the metabolic system. Subjects and coaches were encouraged to ask any questions regarding participation in the study. All subjects were asked to wear standardized clothing (short sleeve cotton t-shirt or mesh practice jersey and shorts) during subsequent experimental trials. Sections 3.4.2 and 3.4.3 discuss the test batteries executed for experimental trials II and I. 


\subsubsection{0-m Shuttle Run}

Created by Leger et.al., the 20-meter shuttle run was intended to test cardiovascular fitness. As continuous aerobic test that corresponds well with the stop and go nature of sports specific activities such as basketball, it has similar characteristics as the children's Fitnessgram PACER test for cardiovascular function. The predicted $\mathrm{VO}_{2}$ max from the 20-m shuttle has demonstrated validity $\left(r=0.84, \mathrm{SEE} 5.4 \mathrm{ml} \cdot \mathrm{kg} \cdot \mathrm{min}^{-1}\right.$ ) when compared to the Balke treadmill protocol to measure $\mathrm{VO}_{2}$ max, as well as reliability $(\mathrm{r}=0.95)$ when tests were conducted one week apart (47).

The 20-m shuttle run employs up to 22 levels, consisting of short running stages within each level. The levels gradually progress in speed and overall intensity as the subject transitions through each phase. To prepare for the test, two lines are established on a basketball court exactly 20 meters apart. A subject would stand behind the first line facing the second line and begin running when instructed by a recording. After reaching the second line, they return to the first line when signaled by a recorded (beep). Following one minute, the sounds reflect an increase in speed, and duration of time between beeps decreases. This continues each minute (level). If a line is not reached in time for a beep, a subject would run to the line, turn, and attempt to catch up with the pace within 2 more 'beeps'. If the subject reached a line before a beep sounded, the subject waited until the beep before starting again. A test was stopped if a subject failed to reach the line for two consecutive beeps. The level at which each subject stopped was recorded. $\mathrm{VO}_{2} \max \left(\mathrm{ml} \cdot \mathrm{kg} \cdot \mathrm{min}^{-1}\right)$ was then predicted for the level obtained on the test using the regression equation validated by Leger and colleagues (47) [Appendix C.1]

EE during this activity was measured simultaneously using the IC (Cosmed K4 $\mathrm{B}^{2}$ ), and Mini (BodyMedia ${ }^{\circledR}$ ) methods. Ratings of Perceived Exertion (RPE) were used as an additional 
measure of intensity during this test and also obtained immediately post exercise. A session RPE was also taken 5 minutes post completion. These techniques are described in section 3.5.1.1 and 3.5.1.2.

\subsubsection{0-Minute Basketball Skills Session}

The 30-minute basketball skills session was created to simulate a high intensity basketball practice or game environment. The drills were selected to reflect the major skills (agility, speed, ball handling, etc.) needed to play the game of basketball, and because of their widespread use in the college basketball setting. The 30-minute basketball specific workout incorporated drills which approach all aspects of the game that include, but are not limited to, sprints, agility, long range shooting, lay-ups, defense, conditioning, etc... (74) (Table 4). Pilot work on the 30minute basketball skills session protocol allowed the principal investigator to determine the order and length of each drill, as well as the 1-minute transition time needed between drills. The pilot protocol was administered to fit college age males and females to determine feasibility of the protocol for subjects and investigator. Each drill in the protocol was separated by a 60 second transition period, with RPE's obtained at the end of each drill. Following the first free throw drill, a 3-minute standing water break occurred (during this time the Cosmed was marked and time was recorded at the start and finish of their break). During the water break subjects were allowed to drink water or Gatorade in a standing position. EE ( $\mathrm{kcal} \cdot \mathrm{min}^{-1}$ and total $\left.\mathrm{kcal}\right)$ during this activity was measured simultaneously using IC (Cosmed K4 $\mathrm{B}^{2}$ ) and the Mini (BodyMedia ${ }^{\circledR}$ ) methods. The Cosmed was marked at the beginning and end of each drill throughout the protocol and during the water break. These techniques will be described in section 3.5.1.1 and 3.5.1.2. 
Table 4: 30-Minute Basketball Skills Session Protocol

\begin{tabular}{|l|l|l||}
\hline PHASE & TIME & PROTOCOL \\
\hline Testing & 5 Minutes & Progressive Defensive Slides \\
\hline & 2 Minutes & Mikan Drill \\
\hline & 2 Minutes & $1 / 2$ Court Speed Lay-Up Drill \\
\hline & 2 Minutes & Victories \\
\hline & 1 Minutes & Free Throws* \\
\hline & 2 Minutes & Medicine Ball Plyometric \\
\hline & 3 Minutes & $1 / 2$ Court Dribbling Drills \\
\hline & 2 Minutes & Toss Out Shooting Drill \\
\hline & 1 Minute & Free Throws \\
\hline
\end{tabular}

- Note:

- There will be a 60 sec transition between each drill

- * Indicates a 3 min standing water break at the end of drill

- RPE was obtained at the end of each drill

\subsection{EXPERIMENTAL TRIAL I AND II}

For Experimental Trials I and II, subjects completed a 20-m shuttle run, and a 30-minute individual basketball specific drill workout. To reduce any thermic effects of food on EE subjects were asked to abstain from caffeine intake and eating for four hours (21). Subjects were also asked to not participate in conditioning sessions for at least 12 hours prior to testing. 
Upon arrival to each experimental trial, anthropometric measures were obtained including height $(\mathrm{cm})$, body weight $(\mathrm{kg})$, Fat free mass $(\mathrm{kg})$, and fat mass (\% and $\mathrm{kg})$. Height (cm) and weight $(\mathrm{kg})$ were measured using a physician's scale. Body composition was assessed using a Tanita (Arlington Heights, IL) Bioelectrical impedance analyzer (BIA) scale. The BIA determines opposition to the flow of an electric current through body tissue, for estimation of total body water, fat-free body mass, and \% body fat (91). Subjects were asked to remove all jewelry in addition to socks. All BIA testing was conducted in "Athletic" mode because of characteristics of the testing sample. Prior to each experimental trial, subjects were familiarized with the RPE scale.

In order to calibrate the mini armband, subjects were asked their birthdate, if they were a smoker, and if they were right handed or left handed. During this time subjects were also asked their playing position, and year in school. Subjects were then fitted with the Cosmed K4 B ${ }^{2}$ unit, and Mini, and escorted to the gymnasium area where they sat in a resting position for 15 minutes to allow the Mini to acclimate to each subject. Following the resting period, subjects engaged in a standardized five-minute dynamic warm-up protocol led by the primary investigator. This included the following exercises: 1) high knees; 2) butt kicks; and 3) walking lunges (Appendix D explains in further detail). Three watches were used for each session to officially track experimental total session time, actual time for start and finish of each drill, and standardized length of each exercise and rest period. This served as a backup to time recorded on the unit. Upon completion of the experimental trial, subjects participated in a five-minute cool-down with a standardized static stretching routine (Appendix I). Time on task was recorded to track transition time, test trial time, and total time to completion for each subject. The 30-minute experimental trial total time required was held consistent for all subjects. 


\subsubsection{Assessments}

\subsubsection{Mini Armband to Assess EE}

Height and weight obtained on testing day were entered into the computer prior to calibration of the Mini. As recommended by the manufacturer, the Mini was worn on the posterior surface of the right upper arm over the belly of the triceps muscle at the midpoint between the acromion and olecranon processes (3). The armband was being held in place with a Velcro strap. As previously mentioned, the armband was placed on the subject's arm and worn while in a seated position for a 15-minute period prior to data collection to allow for acclimation to skin temperature. The armband was time-stamped at the start of the exercise stage and end of session. During each experimental trial, data was stored in the Mini, then downloaded at the conclusion of each activity trial. To calculate energy expenditure, the Mini used accelerometry counts, heat flux, galvanic skin response, skin temperature, and near-armband temperature. Energy expenditure during exercise was computed in 1-min intervals. The exercise data was converted to energy expenditure $\left(\mathrm{kcal} \cdot \mathrm{min}^{-1}\right)$ using a generalized proprietary algorithm in BodyMedia's InnerView $^{\circledR}$ Research Software Version 7.0 (3). The outcome variables $\mathrm{kcal} \cdot \mathrm{min}^{-1}$, total $\mathrm{kcals}$, and METs determined from the InnerView ${ }^{\circledR}$ program were used for data analysis.

\subsubsection{Indirect Calorimetry to Measure EE}

Indirect Calorimetry (IC) was used as the criterion measure of EE. The Cosmed K4 $\mathrm{b}^{2}$ Mobile Metabolic Measuring System (COSMED, Inc., Rome Italy) was used to assess EE during each experimental trial. This system was calibrated prior to each activity period using a known gas volume calibration (3-liter calibration syringe), and gas concentration (reference Gas 5\% $\mathrm{CO}_{2}, 15 \% \mathrm{O}_{2}$ ). Expired gas volumes and concentrations were assessed on a breath- 
by-breath basis, with values averaged at one-minute intervals. Oxygen uptake $\left(\mathrm{ml} \cdot \mathrm{kg} \cdot \mathrm{min}^{-1}\right)$ was converted to $\mathrm{kcal} / \mathrm{min}$ using respiratory metabolism. Energy expenditure was calculated from oxygen consumption with caloric equivalents corrected for the respiratory exchange ratio (RER). Energy expenditure was calculated in $\mathrm{kcal} \cdot \mathrm{min}^{-1}$ using a non-protein caloric equivalent (90). Outcome measures $\left(\mathrm{VO}_{2}, \mathrm{Ve}, \mathrm{VCO}_{2}, \mathrm{kcal} \cdot \mathrm{min}^{-1}, \mathrm{METs}\right.$, total $\left.\mathrm{kcal}\right)$ were obtained each minute of the Experimental Trial I and II.

\subsubsection{Heart Rate}

Heart rate $\left(b \cdot \min ^{-1}\right)$ was measured using a polar monitoring system (Woodbury, NY) from 45-60 seconds of each minute during each experimental trial.

\subsubsection{Rating of Perceived Exertion}

The Adult OMNI Walk/Run Perceived Exertion scale was used to assess the subjects rating of perceived exertion for overall body, chest/breathing, and legs (RPE-O, RPE-C, RPE-L) during Experimental Trial I and II. Defined as "the subjective intensity of effort, strain, discomfort and/or fatigue that is felt during exercise” (64), ratings of perceived exertion (RPE) have been determined to be both reliable and valid (64). Measurement of RPE involves using numerically based category scales that allow a subject to select a number that corresponds to the intensity of their perception of physical exertion. Subjects were familiarized to the scale during the orientation session and prior to each experimental trial. RPE was obtained during the 60-second transition period between each drill during the 30minute basketball skills session. An immediate post exercise RPE was obtained at the end of the 20-m shuttle run test. A session RPE was obtained 5 minutes following the 20-m shuttle run trial and the 30-minute basketball workout (64). 


\subsection{STATISTICAL ANALYSIS}

Statistical analyses were performed using IBM SPSS Statistics (Version 20.0) with level of significance set at $\mathrm{p}<0.05$. Power analysis showed that given a one-tailed alpha of .05 and a correlation (r) of at least .60 between the Cosmed $\mathrm{K} \mathrm{B}^{2}$ and the Mini armband, a sample of 16 participants would result in a power of at least $80 \%$. Descriptive characteristics of subjects are presented as means \pm standard deviations. Data was analyzed separately for each exercise trial. A dependent t-test was used to compare energy expenditure in total $\mathrm{kcals}$, METS, and $\mathrm{kcal} \cdot \mathrm{min}^{-1}$ during both experimental trials. To test the primary hypotheses Pearson correlation coefficients were calculated. The first evaluated the relationship between total energy expenditure (Mini vs. IC) at the end of the 30-minute basketball skills session. The second evaluated the same relationship at the end of the 20-meter shuttle run. Bland Altman plots were also used to assess agreement between IC and Mini. Outcome variables measured at rest and throughout all exercise

trials included: 1) total kilocalories (kcal); 2) calories per minute ( $\left.\mathrm{kcal} \cdot \mathrm{min}^{-1}\right)$; and 3) average METs.

Data was tested for normality and homogeneity. A two-way (method by time (level)) analysis of variance (ANOVA) was performed for the 20-meter shuttle run session using minuteby-minute data during each session. The purpose of this analysis was to examine consistency between the instruments in tracking changes in energy expenditure over the course of a session. 


\subsection{RESULTS}

\subsection{INTRODUCTION}

The specific aim of this investigation was to examine the validity of the BodyMedia ${ }^{\circledR}$ FIT Armband Mini as a measure of energy expenditure of female basketball players during a 30minute period of variable intensity basketball skill exercises in a game-like condition. A secondary aim was to validate the accuracy of the BodyMedia ${ }^{\circledR}$ FIT Armband Mini as a measure

of energy expenditure of female basketball players during a 20-meter shuttle running test of aerobic capacity. This investigation employed a multiple observation cross sectional experimental design. The independent variable for this investigation was energy expenditure (EE) expressed as total kcals; METs; and $\mathrm{kcal} \cdot \mathrm{min}^{-1}$ estimated by the Mini armband. The dependent variable was (EE) expressed as total kcals; METs; $\mathrm{kcal} \cdot \mathrm{min}^{-1}$ determined by the indirect calorimetry (IC) method. The 30-minute basketball skills session results are discussed as a $\% \mathrm{VO}_{2}$ peak estimated from the 20 -meter shuttle run test. Therefore the 20 -meter shuttle results will be presented first. The following sections will provide results of the following: 1) Subject Characteristics (4.2); 2) 20-meter shuttle run test (4.3); 3) 30-minute basketball skill session (4.4). 


\subsection{SUBJECTS}

Sixteen female basketball players (age 18-22 years) participated in this investigation. All subjects attended an orientation/screening session and two experimental testing trials (20-meter shuttle run and a 30-minute basketball skills session). Of the original 15 University of Pittsburgh Division I female basketball players recruited, eight were included in the two experimental trials. Seven players from the University of Pittsburgh team were unable to participate due to coaching restrictions and various injuries. An additional 8 subjects were recruited from the Carnegie Mellon University Division III women’s basketball team to participate in the experimental trials. This investigation included 8 freshman, 4 sophomores, 3 juniors, and 1 senior. Descriptive characteristics of the subjects are presented in Chapter 3 (page 31).

There were no significant differences in descriptive characteristics between the University of Pittsburgh women’s basketball players and the Carnegie Mellon players ( $\mathrm{p}>.05)$.

\subsection{0-METER SHUTTLE RUN TEST}

\subsubsection{Overview}

The objective of the 20-meter shuttle run test was to gain knowledge of each subject's estimated aerobic capacity. The test was also used to evaluate the ability of the Mini to measure EE during continuous exercise of varying intensities. The 20-m shuttle run consists of up to 22 levels, involving short running stages within each level. The levels gradually progressed in speed and overall intensity as the subject transitioned through each phase. A higher level achieved 
suggested a greater level of aerobic fitness for a subject. In this investigation, $\mathrm{VO}_{2} \max$ $\left(\mathrm{ml} \cdot \mathrm{kg} \cdot \mathrm{min}^{-1}\right)$ was predicted for the level achieved during the test using the regression equation validated by Leger and colleagues (47).

\subsubsection{Descriptive Statistics}

Descriptive characteristics relating to the 20-meter shuttle run test are presented in Table 5. Level of completion was converted to a predicted $\mathrm{VO}_{2}$ max $\left(\mathrm{ml} \cdot \mathrm{kg} \cdot \mathrm{min}^{-1}\right)$ using a regression equation (Appendix C). According to American College of Sports Medicine (ACSM) guidelines, subjects averaged “Fair” for aerobic fitness for women aged 18-22 years (2).

Total kcals from IC and Mini were recorded from the start of the test to immediately post exercise. As a means of measuring intensity, peak HR (b· $\mathrm{min}^{-1}$ ) and Session RPE was also recorded. Session RPE was taken 5 minutes post exercise and HR was obtained from the Cosmed data as minute averages.

Table 5 Descriptive Characteristics 20-Meter Shuttle Run Test

\begin{tabular}{|c|c|}
\hline & Mean ( \pm S.D) \\
\hline Level Completed & $6.8 \pm 1.4$ \\
\hline Predicted $\mathrm{VO}_{2} \max \left(\mathrm{ml} \cdot \mathrm{kg} \cdot \mathrm{min}^{-1}\right)$ & $35.7 \pm 4.8$ \\
\hline Total kcal (Cosmed) & $87.2 \pm 25.8$ \\
\hline Total kcal (Mini) & $77.8 \pm 20.6$ \\
\hline Peak Heart Rate $\left(b \cdot\right.$ min $\left.^{-1}\right)$ & $188.6 \pm 8.1$ \\
\hline Session RPE & $5.8 \pm 1.0$ \\
\hline Peak $\mathrm{VO}_{2}\left(\mathrm{ml} \cdot \mathrm{kg} \cdot \mathrm{min}^{-1}\right)($ Cosmed $)$ & $37.29 \pm 4.87$ \\
\hline
\end{tabular}

Values are presented as Mean \pm Standard Deviation. 


\subsubsection{Energy Expenditure}

For the 20-meter shuttle run, a dependent t-test compared energy expenditure from the IC method to total energy expenditure from the Mini (Figure 2; Table 6). There was a significant difference between total kcal determined by IC (87.24 $\pm 25.83 \mathrm{kcal})$ and Mini $(77.83 \pm 20.59$ kcal) ( $\mathrm{p}=.017$ ) for the 20-meter shuttle run test, with the Mini underestimating energy expenditure. EE determined in METs by IC (8.64 \pm 1.16 METs) and Mini (8.23 \pm 0.88 METs) was not significantly different $(p=.194)$ for the 20 -meter shuttle run test. There was also no significant difference between EE for kcal• $\mathrm{min}^{-1}$ determined by IC $\left(11.69 \pm 3.82 \mathrm{kcal} \bullet \mathrm{min}^{-1}\right)$ and Mini $\left(10.39 \pm 1.04 \mathrm{kcal} \bullet \mathrm{min}^{-1}\right)$ for the 20 -meter shuttle run test $(\mathrm{p}=3.01)$.

Error Bars Represent 1 SD

Figure 2 Comparison of EE for 20-Meter Shuttle Run Test 
Table 6 Comparison of METs and Kcal per minute for 20-Meter Shuttle Run Test

\begin{tabular}{|l|c|c|c|}
\hline & Mini & IC & P Value \\
\hline Average METs & $8.23 \pm 0.88$ & $8.64 \pm 1.16$ & .194 \\
\hline kcal•min $^{-1}$ & $10.39 \pm 1.04$ & $11.69 \pm 3.82$ & .301 \\
\hline
\end{tabular}

\subsubsection{Correlations}

Pearson product moment correlation coefficients were calculated to determine the relationship between total kcals from IC and the Mini for the 20-meter shuttle run trial. Results demonstrated a high correlation between total EE in kcals from IC and the Mini for the 20-meter shuttle run test $(r=0.839, p=<.0005, \mathrm{SEE}=14.53 \mathrm{kcal})$ (Figure 3$)$. Results also demonstrated a high correlation between EE in METs $(r=0.859, \mathrm{p}=<.0005)$ and $\mathrm{kcal}^{\circ} \mathrm{min}^{-1}(\mathrm{r}=0.824, \mathrm{p}=.023)$ from IC and the Mini. 


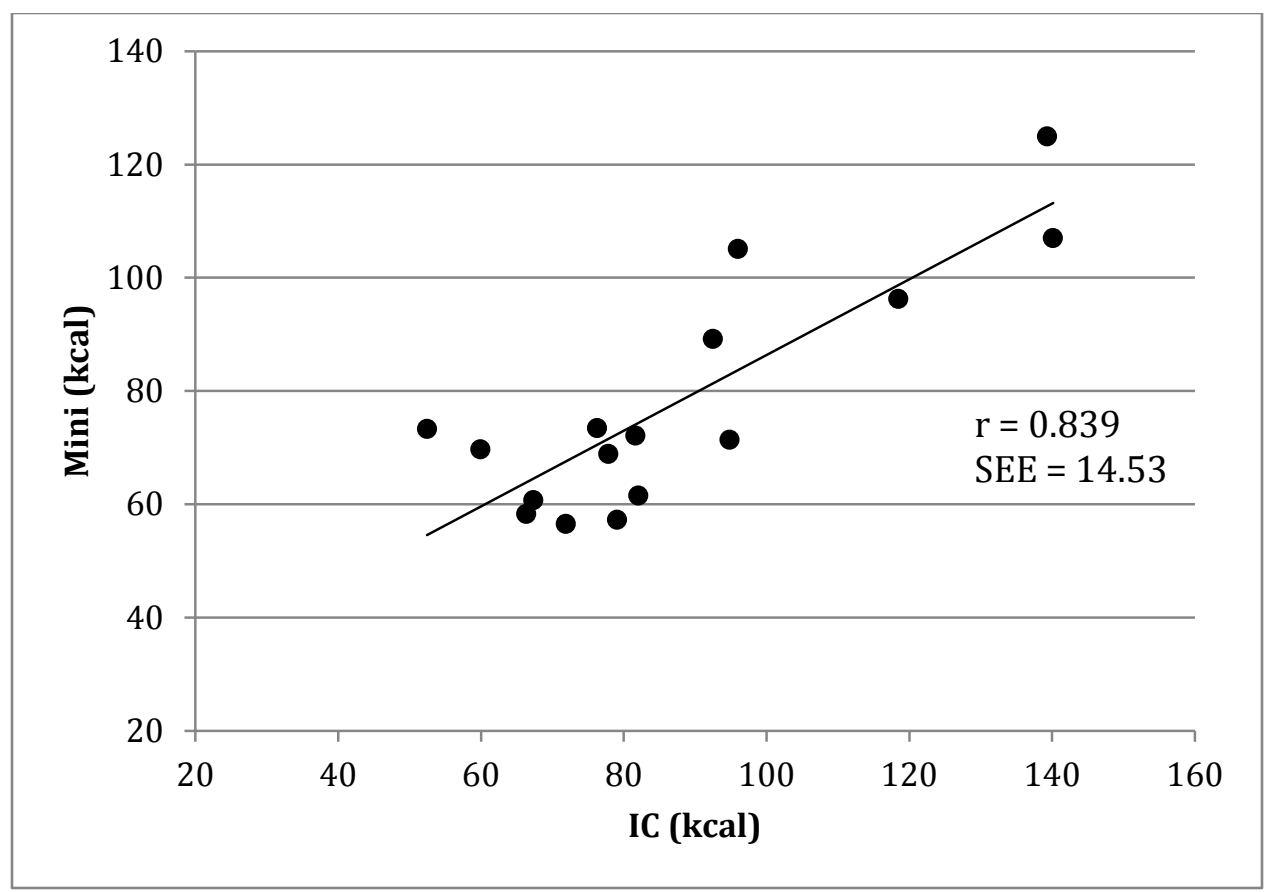

Figure 3 Association Between Mini and IC From 20-Meter Shuttle Run Test

\subsubsection{Bland-Altman}

A Bland-Altman plot analysis technique determines whether two methods agree sufficiently for them to be used interchangeably $(6,7)$. A Bland Altman plot is presented for the 20-meter shuttle run trial in Figure 4 below. As shown in the Bland Altman plot, the mean difference between the Mini and IC values was 9.084 kcals (95\% limits of agreement $=19.076$ and 37.224 kcals). Ideally a mean difference should be closer to a zero value. In this case the upper level of agreement was, 37.224 and large enough to be considered clinically significant. 


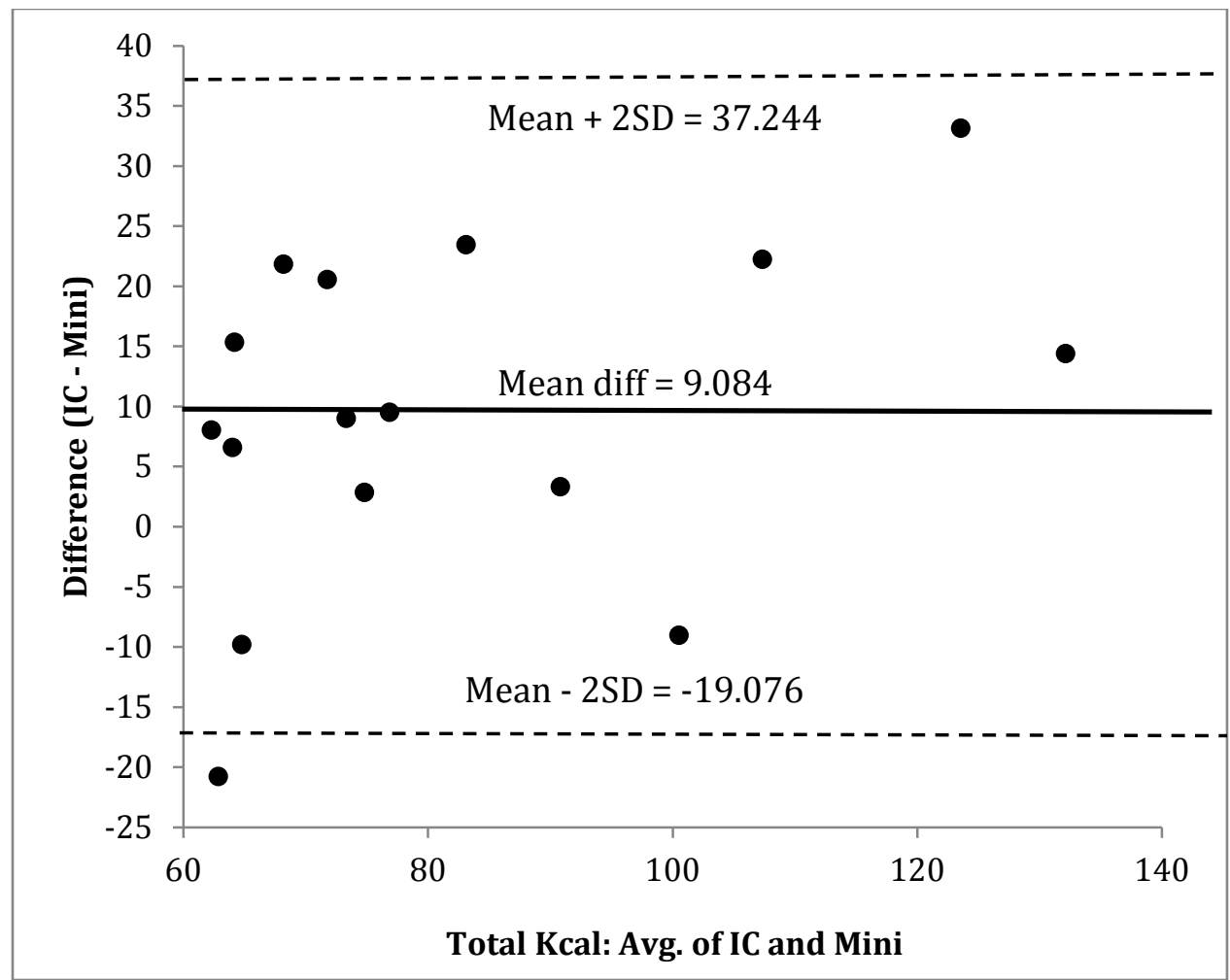

Figure 4 Bland Altman Plots of Agreement Between IC and Mini for 20-Meter Shuttle Run Test

[The dashed line represents the $95 \%$ limits of agreement. The middle solid line represents the mean difference between methods. 95\% limits of agreement $=-19.1$ to 37.2 kcals.]

\subsubsection{Energy Expenditure Estimates Throughout 20-Meter Shuttle Run Test}

Means \pm standard deviations (SD) for energy expenditure in $\mathrm{kcal} \cdot \mathrm{min}^{-1}$ during the 20 -meter shuttle run test are plotted in Figure 5. A two factor (method x level) repeated measures ANOVA assessed differences in EE $\left(\mathrm{kcal} \cdot \mathrm{min}^{-1}\right)$ between measurement devices (IC and Mini) for minuteby-minute comparison throughout the entire exercise session. The ANOVA (APPENDIX K) indicated a non-significant $(\mathrm{p}=.415)$ effect for method and a significant $(\mathrm{p}=<.0005)$ effect for level. Measurement method and level were considered as within-subjects variables in the 
repeated measures ANOVA. In addition, the method by level interaction effect $(\mathrm{F}=83.10, \mathrm{P}<$ 0.05) was significant ( $\mathrm{p}=<.0005$ ). Post Hoc comparisons indicated that the Mini significantly overestimated ( $\mathrm{p}=.000)$ EE (kcals) at level 1 and significantly underestimated $(\mathrm{p}=.000)$ EE (kcals) for all levels 3 and above.

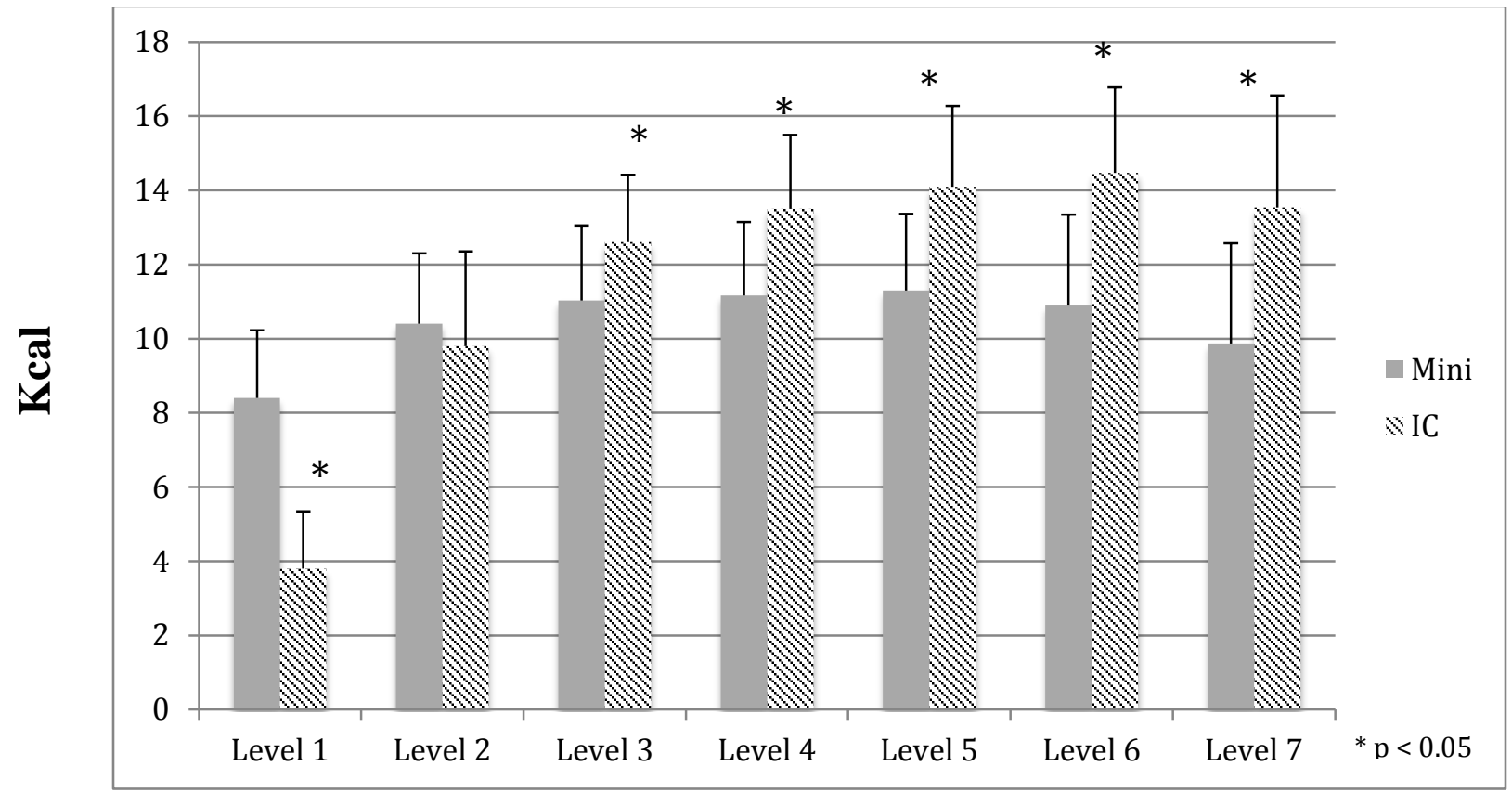

Figure 5 Mean EE Estimates of Mini and IC Compared Throughout 20-Meter Shuttle Run Test

\subsection{MINUTE BASKETBALL SKILLS SESSION}

\subsubsection{Overview}

The 30-minute basketball skills session was created to simulate the high intensity environment of a basketball practice or game. The drills were selected to reflect the major skills (agility, speed, ball handling, etc.) needed to play the game of basketball, and because of their widespread use in 
college basketball. Each drill in the protocol was separated by a 60 second transition period. During this transition period, RPE was obtained for the previous drill and instructions for the next drill were provided. Following the first free throw drill (approximately 15 minutes into the testing session), a 3-minute standing water break occurred. A detailed description of each drill in the 30-minute basketball session is provided in Appendix E.

\subsubsection{Descriptive Statistics}

Descriptive characteristics for the 30-minute basketball session are provided in Table 6. For each drill the mean $\mathrm{VO}_{2}\left(\mathrm{ml} \cdot \mathrm{kg} \cdot \mathrm{min}^{-1}\right)$, \% of $\mathrm{VO}_{2}$ peak (Cosmed measure from 20-meter shuttle run

test), METs, and heart rate $\left(\mathrm{b} \cdot \mathrm{min}^{-1}\right)$ were measured (Table 6). Perceived exertion for the overall body (RPE-O), chest (RPE-C), and legs (RPE-L) were also obtained at the end of each drill (Table 7). 
Table 6 Descriptive Characteristics of 30-Minute Basketball Skills Session

Values are presented as Mean \pm Standard Deviation.

\begin{tabular}{|c|c|c|c|c|c|}
\hline & $\begin{array}{c}\mathrm{VO}_{2} \\
\left(\mathrm{ml} \cdot \mathrm{kg} \cdot \mathrm{min}^{-1}\right)\end{array}$ & $\begin{array}{l}\mathrm{VO}_{2} \text { peak } \\
\text { (From IC) }\end{array}$ & METs & kcal· min $^{-1}$ & HR $\left(b \cdot \min ^{-1}\right)$ \\
\hline Progressive Defensive Slide & $28.9 \pm 3.9$ & $77.5 \%$ & $7.98 \pm .93$ & $10.72 \pm 3.6$ & $165.2 \pm 13.4$ \\
\hline Mikan & $27.8 \pm 3.4$ & $74.6 \%$ & $8.05 \pm .93$ & $11.17 \pm 2.19$ & $170.7 \pm 10.7$ \\
\hline Speed Lay-Up & $28.6 \pm 3.3$ & $76.7 \%$ & $8.32 \pm .91$ & $11.18 \pm 2.61$ & $173.5 \pm 9.2$ \\
\hline Victory & $29.1 \pm 3.6$ & $78 \%$ & $8.41 \pm 1.1$ & $11.58 \pm 2.38$ & $177.8 \pm 8.9$ \\
\hline Free Throw (1) & $19.3 \pm 3.8$ & $51.8 \%$ & $5.9 \pm 1.2$ & $7.96 \pm 1.77$ & $163.9 \pm 15.9$ \\
\hline Medicine Ball Plyometrics & $20.0 \pm 4.1$ & $53.6 \%$ & $6.4 \pm 1.2$ & $8.21 \pm 2.01$ & $155.2 \pm 13.1$ \\
\hline $1 / 2$ Court Dribbling Drills & $25.0 \pm 6.4$ & $66 \%$ & $7.7 \pm .86$ & $10.14 \pm 2.02$ & $172.5 \pm 10.8$ \\
\hline Toss Out Shooting Drill & $24.9 \pm 2.7$ & $66.8 \%$ & $7.2 \pm .86$ & $9.65 \pm 1.7$ & $172.1 \pm 10.8$ \\
\hline Free Throws (2) & $19.1 \pm 3.2$ & $51.2 \%$ & $5.2 \pm .86$ & $6.79 \pm 1.58$ & $144.1 \pm 57.4$ \\
\hline
\end{tabular}


Table 7 RPE of 30-Minute Basketball Skills Session

\begin{tabular}{|l|c|c|c|}
\hline \multicolumn{3}{|c|}{ Ratings of Perceived Exertion } \\
\hline Drills & Overall & Chest & Legs \\
\hline Progressive Defensive Slide & $5.7 \pm 1.3$ & $5.4 \pm 1.6$ & $5.5 \pm 1.5$ \\
\hline Mikan & $5.1 \pm 1.5$ & $5.0 \pm 1.8$ & $4.8 \pm 1.8$ \\
\hline Speed Lay-Up & $6.6 \pm 1.2$ & $6.4 \pm 1.6$ & $6.4 \pm 1.4$ \\
\hline Victory & $7.8 \pm 1.0$ & $7.7 \pm 1.3$ & $7.5 \pm 1.5$ \\
\hline Free Throw (1) & $2.5 \pm 2$ & $2.6 \pm 1.9$ & $2.3 \pm 1.9$ \\
\hline Medicine Ball Plyometrics & $3.7 \pm 1.7$ & $3.3 \pm 1.8$ & $2.8 \pm 1.9$ \\
\hline $1 / 2$ Court Dribbling Drills & $5.3 \pm 1.4$ & $4.9 \pm 1.9$ & $5.1 \pm 1.8$ \\
\hline Toss Out Shooting Drill & $3.9 \pm 1.1$ & $3.6 \pm 1.7$ & $3.7 \pm 1.4$ \\
\hline Free Throws (2) & $1.7 \pm 1.4$ & $1.9 \pm 1.7$ & $1.6 \pm 1.5$ \\
\hline Session & $5.7 \pm 1.4$ & $6.0 \pm 1.2$ & $5.3 \pm 1.5$ \\
\hline
\end{tabular}

Values are presented as Mean \pm Standard Deviation.

\subsubsection{Energy Expenditure}

For the 30-minute basketball skills session a dependent t-test was performed to compare EE between the IC and Mini. Total kcals of the 30-minute basketball session were significantly greater in IC compared to the Mini method $(\mathrm{p}=<.0005)$ (Figure 6). Therefore, in the 30-minute basketball skills session the Mini (179.56 $\pm 35.51 \mathrm{kcal})$ underestimated EE compared to the IC method (236.27 $\pm 46.75 \mathrm{kcal})$. EE in METs determined by IC (7.35 \pm .79 METs) and Mini (5.94 \pm 0.42 METs) were significantly different $(p<.05)$ for the 30 -minute basketball skills session. There was also a significant difference between EE in kcals per minute determined by IC (9.74 \pm $\left.1.78 \mathrm{kcal} \bullet \mathrm{min}^{-1}\right)$ and Mini $\left(7.08 \pm 1.57 \mathrm{kcal} \bullet \mathrm{min}^{-1}\right)$ for the 30 -minute basketball skills session (p $=<$.0005), with the Mini underestimating energy expenditure. 


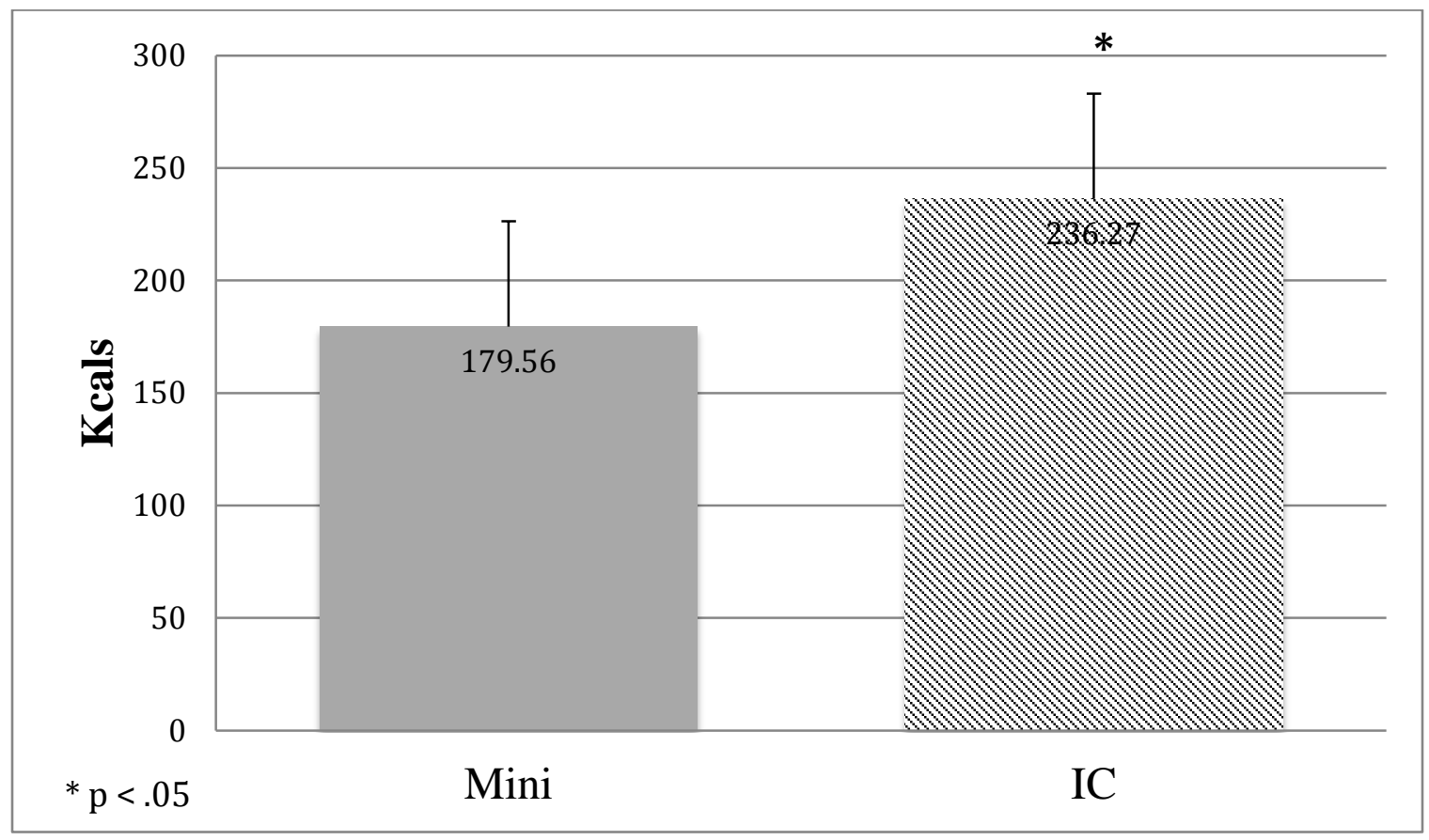

Figure 6 Comparison of EE (kcals) for 30-Minute Basketball Skills Session

Table 8 Comparison of METs and $\mathrm{kcal} \cdot \mathrm{min}^{-1}$ for 30-Minute Basketball Skills Session

\begin{tabular}{|c|c|c|c|}
\hline & Mini & IC & P value \\
\hline Average METs & $5.94 \pm 0.42 *$ & $7.35 \pm .79$ & .000 \\
\hline $\mathrm{kcal} \cdot \mathrm{min}^{-1}$ & $7.07 \pm 1.57 *$ & $9.74 \pm 1.78$ & .000 \\
\hline
\end{tabular}

Values are presented as Mean \pm Standard Deviation.; ${ }^{*} \mathrm{p}<.05$

\subsubsection{Correlations}

Pearson product moment correlation coefficients determined the relationship between total EE (kcals) from IC and the Mini for the 30-minute basketball trial. Results demonstrated a high 
correlation between total EE for kcals from IC and the Mini for the 30-minute basketball skills session $(r=0.833, p=.000, \mathrm{SEE}=26.74$ kcals $)$. Results also demonstrated both high and modest correlations between total EE in METs $(r=0.861, \mathrm{p}=.000)$ and $\mathrm{kcal} \cdot \mathrm{min}^{-1}(\mathrm{r}=0.634, \mathrm{p}=.000)$.

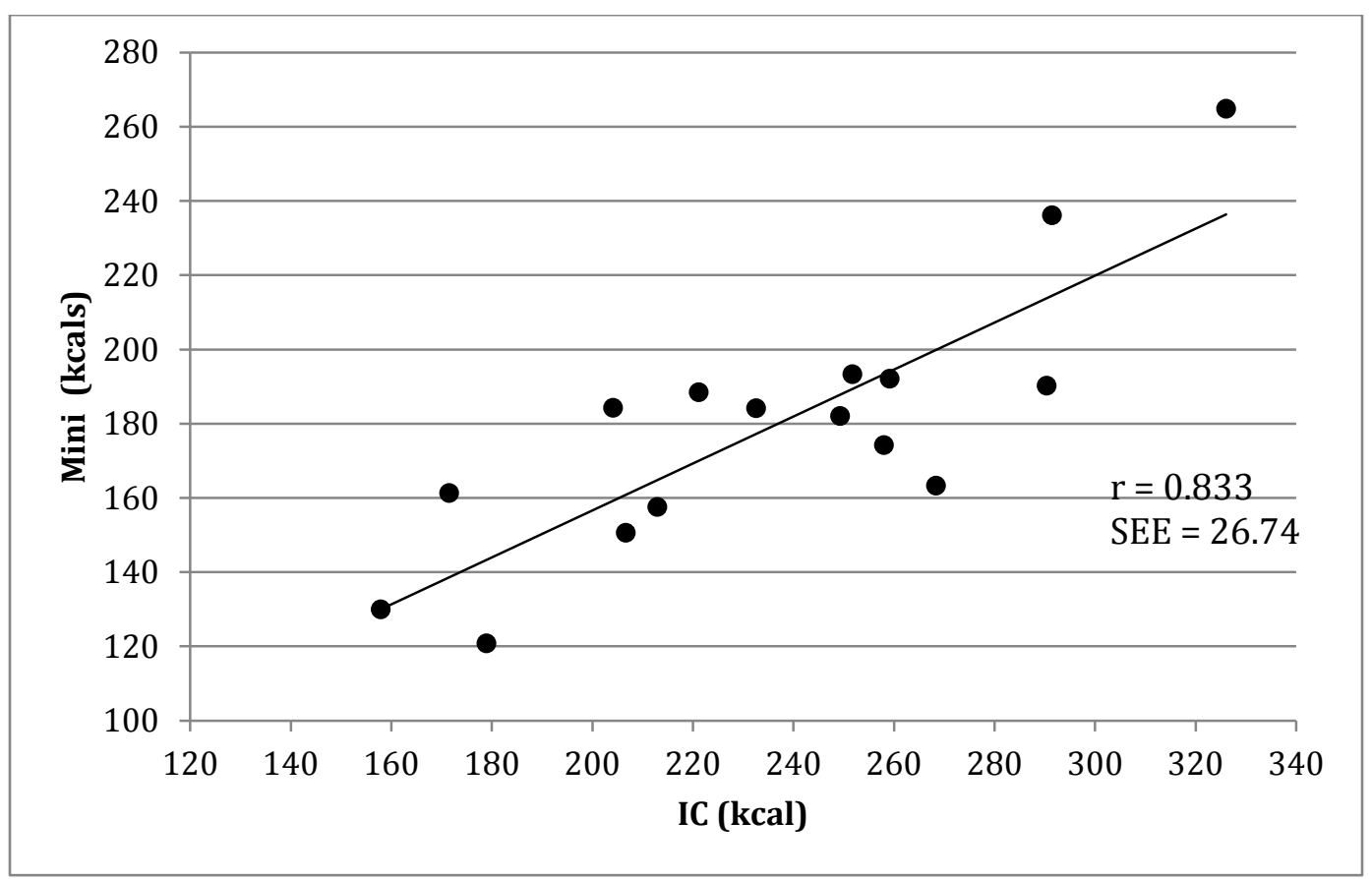

Figure 7 Comparison of Total EE (kcals) for 30-Minute Basketball Skills Session

\subsubsection{Bland-Altman}

A Bland Altman plot is presented for the 30-minute basketball skills session in Figure 8 below. According to the Bland Altman analysis, the mean difference between the Mini and IC values was 57.713 kcals (95\% limits of agreement $=4.59$ and 108.8 kcals). There appears to be large limits of agreement with the upper limit at almost 109 kcals. This would be clinically important when considering the interchangeability of the two methods. 


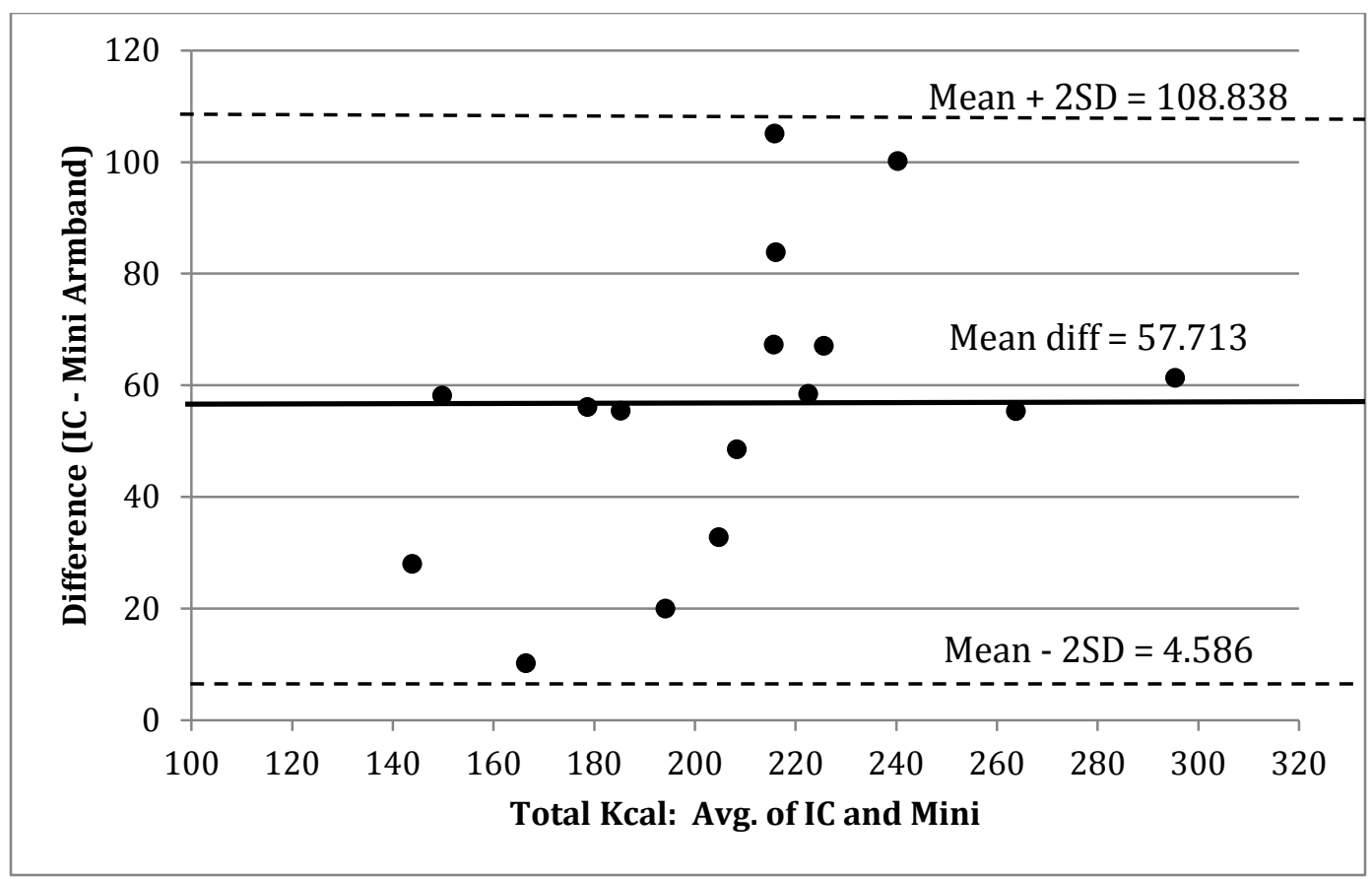

Figure 8 Bland Altman Plots of Agreement Between IC and Mini For The 30-minute Basketball Skills Session

[The dashed line represents the $95 \%$ limits of agreement. The middle solid line represents the mean difference between methods. 95\% limits of agreement $=4.59$ to 108.84 kcals.]

\subsection{SUMMARY}

The primary aim of this study was to examine the validity of the Mini to assess EE during variable intensity basketball skill and game-like conditions in female basketball players. Results from this investigation demonstrated that the Mini significantly underestimated total kcals of the 30-minute basketball skills session. However, results demonstrated a strong relationship between energy expenditure from IC and the Mini for the 30-minute basketball skills session ( $r=0.833$, $p$ $=<.0005$, SEE $=26.74$ kcals) . 
A secondary aim of this study was to examine the accuracy of the Mini to assess EE during a 20-meter shuttle run test in female basketball players. Similar to the 30-minute basketball skills session results showed that the Mini underestimated energy expenditure during the 20-meter shuttle run test. A strong relationship between energy expenditure from IC and the Mini for the 20-meter shuttle run test was shown $(r=0.839, p=.000$, SEE$=14.53 \mathrm{kcal})$. Bland Altman plots also indicated that the majority of data fell within the $95 \%$ limits of agreement for both trials. However, the limits encompass a very wide range, showing a lower level of agreement the more kcals the subject expended. The mean difference shown on the Bland Altman plots for each trial are also high at 9.08 and 57.7 kcals. Ideally the mean difference should be close to the zero point. A method x level ANOVA was also performed to examine the consistency between the instruments in tracking changes in energy expenditure over the course of the 20-meter shuttle run test. The ANOVA showed that the Mini underestimated EE over a course of the 20-meter shuttle run test, particularly at higher (more intense) levels. Chapter 5 will discuss factors explaining the discrepancies between the Mini and IC methods for both experimental trials. Chapter 5 will discuss factors explaining the discrepancies between the Mini and IC methods for both experimental trials. 


\subsection{DISCUSSION}

\subsection{INTRODUCTION}

Although previous studies have examined the validity of the SenseWear Pro Armband during low to moderate intensity activities, few have included high intensity activities, and none have included intermittent activity. The present study was the first to examine the validity of the Mini to measure EE in female basketball players at intermittent intensities. The primary purpose of this study was to validate the accuracy of the Mini in measuring EE of female basketball players during a 30-minute variable intensity basketball skill and game-like condition.

In the present investigation, total EE was underestimated in each experimental trial. Yet for both trials, EE was highly correlated between the Mini and IC methods of measurement. Together, these findings may have important implications when monitoring EE in athletes of the intermittent nature.

There are several mechanisms that may explain the underestimation of EE. Originally, the Mini employed generalized algorithm for physical activity energy expenditure developed using adult formulas for estimating EE during various modes of exercise (31, 22, 39, 40, 78, 43, 51, 52). More recently, these algorithms were revised based on the evaluation of subjects engaged in continuous high intensity exercise $(43,22)$. In the present study, using these formulas and algorithms to predict EE during intermittent activity may have increased the likelihood of 
error in EE estimations. This and other factors will be examined more closely as they relate to the underestimation of EE during the specific exercise modality evaluated in the present investigation. The following discussion will include: 1) total EE, minute-by-minute EE, and correlations for the 20-meter shuttle run test; 2) total EE, and correlations for the 30 minute basketball skills session; 3) factors explaining differences; 4) limitations; and 5) recommendations for future research.

\subsection{0- METER SHUTTLE RUN TEST}

\subsubsection{Energy Expenditure}

The Mini significantly underestimated total $(9.4 \pm 14.1 \mathrm{kcal})$ EE compared to IC for the 20-meter shuttle run test in female basketball players. This discrepancy is noteworthy considering the average total kcal for the entire test was $87.2 \mathrm{kcal}$. Intraclass correlations relating EE from the two devices for the 20-meter shuttle run test were $r=0.839$ (SEE= $14.53 \mathrm{kcal})$. These strong associations for total kcals were similar when EE was expressed as METs and $\mathrm{kcal} \bullet \mathrm{min}^{-1}(\mathrm{r}=$ $0.859, \mathrm{r}=0.824$ ) between the two devices. These results are consistent with a study by Jakicic et al., where the SenseWear ${ }^{\circledR}$ Pro 2 Armband (SWA) was strongly related to IC yet significantly underestimated total energy expenditure during a 30 minute treadmill protocol in an adult female and male sample (39). Similarly, a study by Drenowatz and Eissenmann found the SenseWear ${ }^{\circledR}$ Pro 2 Armband significantly underestimated total energy expenditure during 10 minute bouts of treadmill running at varying intensities (22). A study by Benito and colleagues also showed an underestimation of EE by the SWA when EE was measured during a higher intensity of 
resistance training (5). In the present study, a Bland Altman plot indicated that most subjects fell within the $95 \%$ limits of agreement. However the range of difference was very wide and showed a larger average difference the more kcals a subject expended. The mean difference in the Bland Altman plot should ideally be at zero, for this experimental trial it was 9.08 kcals.

Despite finding a significant underestimation of EE by the Mini for the 20-meter shuttle run, analysis of data showed significant correlations $(r=0.839$, SEE $=14.53$ kcals, $p<0.05)$ between the Mini and IC for the 20-meter shuttle run test. Several studies have demonstrated similar findings (31, 22, 39, 40, 78, 43, 51, 52). Drenowatz and Eisenmann (22) found moderate to strong correlations between EE measured by IC and EE estimated from the SenseWear Pro Armband $(\mathrm{r}=0.66)$ during 10 -minute bouts of treadmill running. In an early validation study executed by BodyMedia, Liden and colleagues examined the accuracy and reliability of the SenseWear Pro armband compared to IC using various modalities and rest. Accuracy levels of $90 \%$ and greater was shown with reliability exceeding $90 \%$ in all conditions $(51,52)$. These initial studies contributed to the proprietary algorithms developed to predict energy expenditure. Since then several studies have found strong agreement between the SenseWear armband and various methods of measuring indirect calorimetry (31, 22, 39, 40, 78, 43, 51, 52).

\subsubsection{Minute-By-Minute Energy Expenditure}

Analysis of variance examined the effect of method, 20-meter test level, and the interaction between the two on energy expenditure. There was no significant difference between Mini and IC methods $(\mathrm{p}=.415)$. There was a significant $(\mathrm{p}=<.0005)$ effect for levels and a significant $(\mathrm{p}$ $=<.0005$ ) interaction between test level and method. The Mini underestimated EE during the 
20-meter shuttle run test compared to IC in female basketball players. As the test level increased (representing greater intensity), the degree of underestimation also increased. The underestimation of EE occurred for all levels except 1 and 2. During level 1 the armband significantly overestimated EE. While the SWA has been shown to accurately estimate energy expenditure at low $(78,31,39)$ and moderate $(78,39,31)$ exercise intensities, results of this study agree with previous studies investigating the validity of the SWA during high intensity exercise $(78,31,39)$. In a study on male endurance athletes by Koehler et al. (43), the SWA adequately assessed energy expenditure during running at $8.5 \mathrm{~km} / \mathrm{h}(5.3 \mathrm{mi} / \mathrm{h})$, but significantly underestimated energy expenditure at higher speeds. Consistent with previous studies, the present study appeared to underestimate EE at higher intensities, and the higher the intensity, the greater the level of underestimation. The mini also overestimated EE during level 1 of the test; this was possibly due to the mini being placed on subject for 15-20 minutes prior to the Cosmed fitting. This first minute of underestimation may have been due to the Cosmed acclimating to the subject.

\subsection{0-MINUTE BASKETBALL SKILLS SESSION}

Unlike the consistent running modality of the 20-meter shuttle trial, the 30-minute basketball skills session incorporated several different drills with varying intensities and rest time. From examination of the data, it appeared that minute-by-minute EE from analysis of variance was unstable to be conducted. This was due to the fact that a steady state was not always achieved within each drill, nor was a permanent steady state achieved throughout. Also due to the protocol at any given time point during the 30-minute basketball skills session not all participants were 
engaged in the same activity. Therefore, a clear division of level or intensity was not established and considered highly variable. Thus the analysis only evaluated total EE for the 30-minute basketball skills session.

\subsubsection{Energy Expenditure}

The Mini significantly underestimated (56.7 $\pm 26.1 \mathrm{kcal}$ ) EE compared to IC during the 30minute basketball skills session in female basketball players. The underestimation of EE was similar when EE was expressed as METs and $\mathrm{kcal} \bullet \mathrm{min}^{-1}$. Significant correlations of total EE were found between the two devices for the 30-minute basketball skills session $(r=0.833$, SEE $=$ 26.74 kcals, $\mathrm{p}<0.05$ ). In the present study, a Bland Altman plot indicated that most subjects fell within the 95\% limits of agreement. However, the large limits of agreement with the upper limit being almost 109 kcals would be too vast to allow these methods to be easily interchangeable. Also, the mean difference in the Bland Altman plot should ideally be at zero, for this experimental trial it was $57.7 \mathrm{kcals}$.

Similarly, Sorjic et al., (76) found strong correlations between EE measured by IC and EE estimated from the SenseWear Pro Armband ( $r=0.81)$. Koehler and colleagues (43) reported a significant correlation $(\mathrm{r}=0.73$ ) between the SWA and EE measured by the doubly labeled water method (DLW) for daily total energy expenditure. The potential factors responsible for the underestimation will be discussed in the following Section 5.4. 


\subsubsection{Ratings of Perceived Exertion}

Ratings of perceived exertion during the 30-minute basketball skills session indicated that drills ranged from a 1.6 to 7.8. Perceived exertion for the overall body (RPE-O), chest (RPE-C), and legs (RPE-L) were obtained at the end of each drill. It appears that the Speed Lay-Ups and the Victory drill were the highest perceived intensity activities with free throws being the lowest perceived intensity. This is in line with the observed peak $\mathrm{VO}_{2}$ for these drills, Speed Lay-Ups and Victories were the highest intensity drills and Free throws were the lowest. The Session RPE for this experimental trial was 5.7,6.0, and 5.3 for overall, chest, and legs respectively. This appears to be similar to the average of the actual RPE's recorded throughout the entire session. There is currently no published data which incorporates the Omni Walk/Run perceived exertion scale to examine exercise intensity or performance in basketball players at the collegiate level.

\subsection{POTENTIAL FACTORS CONTRIBUTING TO MINI VS IC DIFFERENCES}

The present findings suggested an underestimation of total EE for the 20-meter shuttle run trial and the 30-minute basketball skills trial. Although only EE was compared by level in the 20meter shuttle run trial compared EE throughout, it appeared that EE was underestimated at intensities at or above 7.4 METs. In our subjects this occurred at approximately level 3, which corresponded with $72 \%$ of the mean $\mathrm{VO}_{2}\left(\mathrm{ml} \cdot \mathrm{kg} \cdot \mathrm{min}^{-1}\right)$ peak values observed during the 20meter shuttle run test. Several underlying mechanisms may assist with understanding the factors responsible for the underestimation of EE derived from the Mini. 


\subsubsection{Generalized Algorithms}

It is important to note that exercise specific algorithms of the intermittent nature were not available for the present investigation. Specifically, algorithms have not been developed for athletic populations, or exercises of the intermittent nature. The intent was to examine the validity of the commercially available (generalized) algorithms (version 7.0) for the Mini device. A 2006 paper published by BodyMedia explained that each of the Mini’s physiological sensors contributes an equal proportion towards the determination of EE (3). However, it seems that given a particular modality, one designated type of sensor may play a greater role in the determination of EE than another. For example, running is an activity highly dependent on locomotion thus, a greater reliance could be placed on the accelerometers during running. Jakicic et al., found that when proprietary exercise-specific algorithms were applied to their data, energy expenditure estimation improved (39). That is, when exercise specific algorithms were used, there were no significant differences in total EE between the SenseWear Pro Armband (SWA) and the respiratory metabolic system for walking, cycling, stair stepping, and arm cranking (39). Therefore, information regarding the nature of activities being performed is vital to accurately estimate EE using a device such as the Mini. For basketball, patterns involving agility and explosive multi-directional movements ought to be considered.

While providing modality specific information may be ideal, there are practical issues related to using activity-specific prediction equations. If an individual user needs to manually select an activity-specific algorithm each time they engage in a different activity, the procedure could prove cumbersome and lead to intra-individual error. Alternatively, if exercise specific algorithms were available to the armband device and accompanying software without relying on 
frequent user input, the use of activity monitors could be more effective in estimating EE in a free living environment. This could help to improve the EE estimate of intermittent activity throughout sports specific training. Specifically, this could allow coaches and players to have an accurate account of the amount of energy expended during a basketball scrimmage or game.

\subsubsection{Body Heat Sensor Input}

Evidence has shown that there is a delayed response between when heat is first produced during PA and when a BodyMedia monitor detects changes in body temperature (3). Of the two body heat sensors in the Mini, the heat flux sensor responds more rapidly than the galvanic skin response (GSR), which is delayed by several minutes (3). BodyMedia reported that body heat sensors provide a more accurate response to protocols lasting eight minutes or longer (3). The protocol for the 30-minute basketball skills session was divided into drills, none of which lasted longer than 5 minutes. While subjects did have a one-minute transition period to the next drill, they still maintained an elevated metabolic rate, and did not return to their physiological baseline. In all, these periods of activity may not have provided the body heat sensors enough time to accurately provide data reflective of metabolic heat production in muscle. In addition, some of the drills (free throws) are classified as low intensity exercise where metabolic heat production would be less. Given a delay in response to the body heat sensors and the lower body heat generated during low intensity exercise, input from the body heat sensors may not have reflected the actual energy cost associated with these exercises. In addition it should also be considered that sweat production increases with higher intensity exercise, and also increases with higher trained individuals (81). Due to this investigation taking place in a free living environment certain conditions were variable from session to session such as temperature and 
relative humidity. The Cosmed was calibrated to include humidity. However such changes may have impacted sweat rate higher sweat rates could act as a barrier to the conduction pathways on the Mini sensors. This could lead to measurement errors in the heat sensors (22). All subjects wore similar clothes (tank tops and shorts), so evaporation rate was not compromised.

\subsubsection{Accelerometer}

The Mini uses accelerometers along with physiological monitors to estimate EE. Accelerometers have been shown to underestimate energy expenditure at higher intensities $(34,43)$. In general, accelerometry methods do not account for stride length changes as walking speed varies, leading to underestimation of energy expenditure, particularly at higher speeds (1). In addition, accelerometers are shown to inaccurately assess energy expenditure during incline walking (1), reported in the SWA as well (31). During the 20-meter shuttle run the movement patterns are the same. While in the 30-minute basketball skills session the EE discrepancy was larger and the movement patterns considered very diverse. It seems that during the 30-minute basketball skills session the Mini cannot overcome these limitations despite using heat-related measurements in addition to accelerometry (1). Furthermore, accelerometry has been shown to be less accurate at detecting vertical movements (76). In basketball this could be problematic due to its multi-planar movements. The sport of basketball incorporates jumping side to side shuffling, and quick movements in any direction.

\subsubsection{Protocol}

This investigation used indirect calorimetry as the dependent variable that quantified energy 
expenditure. The protocol for each experimental session may have included some anaerobic pathways, which may not have been detected by the indirect calorimetry method (76). If possible to detect, this may have made the underestimation even greater. Although the aerobic and anaerobic aspects of basketball are considered the nature of the sport, this may have contributed to possible discrepancies between the two measures. Also, the protocol for this investigation included various intensity basketball specific drills with little rest. This allowed for no achievement of steady state throughout the 30-minute basketball skills session. This closely reflected the intermittent nature that fluctuates from low to high intensity throughout an entire practice or game. Previous studies have implemented protocols that will allow for steady state to be established (31, 22, 39, 40, 78, 43, 51, 52, 76). The present study appears to be the first deliberate protocol, which did not allow for a steady state to occur. Therefore, it should be considered if the Mini's ability to estimate EE during both Trial I and II was altered by the qualities of the protocol used in the present investigation.

\section{$5.5 \quad$ STRENGTHS}

1. The protocol for this investigation included a 30-minute basketball skills session considered very sports specific, and mimicked game-like activities that included the most widely used drills in basketball (dribbling, defensive slides, shooting, etc.). The pilot work conducted using fit college males age 18-23 allowed for a well-organized protocol with smooth transition periods between drills.

2. This investigation took place in a free-living environment, with activities not as tightly controlled compared to a traditional laboratory setting. Several aspects of the study 
were controlled, and included: 1) preparation of subjects; 2) clothing; 3) drill length; 4) warm-up; 5) cool-down; and 6) water break.

3. The portable nature of the Cosmed device allowed for a true sports specific protocol to be executed. The unique design of the Cosmed allowed subjects to move freely throughout. The Mini armband also allowed for free movement, and did not impede movements at any point of the protocol.

4. Characteristics of physiological measures (Table 7) appear to indicate that the protocol demonstrated that subjects who engaged in a 30-minute session performed a large proportion of the trial aerobically with subjects on average performing between a $51 \%$ and $78 \%$ of their measured $\mathrm{VO}_{2}$ peak. The Ratings of Perceived Exertion measures support this, however a large range across drills was observed (1.7-7.8 using OMNI scale). It is important to note that several drills (1/2 court dribbling, Mikan, speed layups, and victories) suggested subjects were working at or above a group normalized RPE corresponding to the ventilatory breakpoint of 5-6 on the Omni Scale (64) This suggest the 30-minute protocol is an ideal aerobically dominant stimulus.

5. The protocol for the 30-minute basketball skills session incorporated both the aerobic and anaerobic needs that reflected all energy systems used during the sport of basketball. The protocol included a diverse range of intensities, durations, and multiplanar movements.

\section{$5.6 \quad$ LIMITATIONS}

This investigation is not without limitations. These limitations should be taken into consideration 
when interpreting the results of this study, as they may affect the application of the findings. Moreover, future studies should address these potential limitations.

1. This investigation did not include a resting measure comparison. Previous studies have compared resting EE in normal weight adults and children (31), however this has not yet been examined in athletes. Furthermore, reliability of the Mini could have been determined with repeated trials at rest.

2. This investigation was executed in a free-living environment to allow it to be as close to a game like situation as possible. Due to this, certain conditions were variable from session to session such as temperature and relative humidity. The Cosmed was calibrated to include humidity. However such changes may have impacted sweat rate, resulting in an inability of heat sensors to accurately measure in the Mini.

3. This investigation took place during the college basketball post-season. Although all athletes agreed to participate in this study, some may have been unmotivated to perform their best due to burnout from the season or lack of interest in study results. While this may not have influenced the sensitivity of the devices directly, it was still important to provide maximal performance effort on all trials.

4. This investigation did not include the measurement of energy expenditure post exercise (EPOC). This may be helpful in determining the complete amount of energy expended during variable intensity game-like conditions (43). A study done by Frunin and Rankin showed that the SenseWear Pro armband accurately estimated post treadmill EE when compared to IC (31). 
5. This investigation showed that the Mini significantly overestimated EE during the first minute of the 20-meter shuttle run test when compared to IC. This may have been due to the Mini being on subjects for several minutes prior to the Cosmed for the acclimatization period. This could have caused the statistically significant overestimation of EE by the Mini during the first minute of the 20-meter shuttle run test.

\subsection{APPLICATION}

Results of this investigation are the initial step in examining the Mini armband as a means of accurately estimating EE in an athletic population. The outcomes of the present study are the first to provide athletes, coaches, and trainers with an estimate of the caloric demands of collegiate female basketball players during simulated game-like conditions. In addition, results of this study express the energy demands associated with anaerobic and aerobic training drills and sets. This will provide insight to coaches when considering metabolic demands of specific workout components, and contribute to improved workout designs and the assessment of recovery needs. Through the quantification of energy requirements, the armband may assist with the determination of caloric needs to properly monitor and help to maintain body composition throughout a competitive season. It could also provide insight as to the intensity level. For example, a combination of Mini, heart rate, and perceived exertion monitoring can provide valuable information on "how hard" an athlete is working and/or if this should be adjusted throughout a season.

This was the first validation study conducted using the Mini to estimate EE during various intensity game-like activities in female basketball players. Results of this study indicated 
that for the 30-minute basketball skills session and the 20-meter shuttle run test, the Mini significantly underestimated EE. These findings impose limitations on the use of the Mini during variable intensity activities. Further research and refinement on the Mini algorithms are needed before this device can be used to estimate EE during variable intensity exercise in a free-living environment. It is proposed that data from this investigation could potentially assist BodyMedia in developing exercise specific algorithms for intermittent activities that are a standard feature for the armband system. A valid physical activity monitor, such as the Mini, that is able to accurately measure physical activity EE should be studied further to answer long-standing questions about energy needs, and requirements in athletes whose sport requires variable intensity and intermittent activity.

\subsection{RECOMMENDATIONS FOR FUTURE RESEARCH}

Based on the findings of this investigation, future research on the validation of the Mini to measure EE could include the following:

1. This investigation used the proprietary algorithm in version 7.0 of the SenseWear Professional software provided with the BodyMedia ${ }^{\circledR}$ FIT Armband Mini. Future research should examine whether additional refinements in the algorithm may improve the ability to accurately estimate energy expenditure in athletes of the intermittent nature. A correction factor should also be considered as part of a new algorithm to eliminate error during protocols that incrementally increase in intensity.

2. The present investigation included 16 female basketball players. Future research 
should increase sample size and include males so a gender comparison may be conducted.

3. The novel design of this study was original and highly sports specific in nature. This can serve as an initial step for future investigations with basketball players. This may also serve as a foundation whereby EE may be measured in other sports of the intermittent nature (volleyball, soccer, lacrosse, etc.)

4. The present study did not look at EE during recovery (EPOC). Future research should look at post exercise EE following game-like conditions to better understand the complete energy demands of athletes, particularly in sports of the intermittent nature.

5. This investigation should be conducted again in a similar manner to cross validate the Mini during intermittent activity, and explore reliability of this device.

6. A future investigation should include the measurement of skin folds to examine if anthropometric measures play a role in error associated with heat sensors.

\subsection{CONCLUSION}

A primary finding of this investigation demonstrated that the Mini significantly underestimated total EE for the 20-meter shuttle run test and the 30-minute basketball specific skills session when compared to indirect calorimetry. The findings from this investigation do not support the primary hypothesis or the secondary hypothesis that energy expenditure measured by the BodyMedia ${ }^{\circledR}$ FIT Armband Mini during variable intensity game-like activity would be similar to 
EE measured by indirect calorimetry. The primary and secondary aim of this investigation were not supported due to the armband significantly underestimating total EE during the 20-meter shuttle run test and the 30-minute basketball skills session by $9.4 \pm 14.1$ kcals and $56.7 \pm 26.1$ kcals respectively, when compared to indirect calorimetry. However a secondary finding of the study does support that significant correlations occurred between the Mini and IC for both the 20-meter shuttle run test and the 30-minute basketball skills session.

The results are consistent with previous research by Drenowatz and Eisenmann (22), who showed that the SenseWear Pro Armband significantly underestimates energy expenditure in endurance trained athletes working at 10 MET's or above. Similar to the results of Drenowatz and Eisenmann, Koehler et al., found the SenseWear Pro Armband to consistently underestimate energy expenditure at higher running speeds (43). The SenseWear Pro Armband significantly underestimated energy expenditure for most exercise intensities, and the underestimation increased as exercise intensity increased (43). Similarly, the findings of this current investigation demonstrated that the armband underestimated total energy expenditure for both sessions and the underestimation increased as exercise intensity/level increased. The present findings suggest that the possible mechanisms underlying the underestimation of EE are complex but may include: 1) the use of generalized exercise algorithms to predict all types of physical activity; 2) the delay in detecting body heat transfer to the skin; and 3) an inaccuracy of the accelerometer during certain basketball related movements. This may require that additional research be conducted to allow for refinement of the prediction algorithms applied to subjects. Although the present investigation is not without limitations, this is the first study to investigate the accuracy of the armband to estimated EE in variable intensity exercise. It is also the first study to examine the accuracy of the armband during activities that simulate game-like situations for athletes. These 
findings are an important first step in validating the Mini technology for use in sports of an intermittent nature. 


\section{APPENDIX A}

\section{SENSEWEAR MINI ARMBAND}

\section{Galvanic Skin Response}

When you sweat, your skin becomes more electrically conductive. This measurement helps to see how active you are.

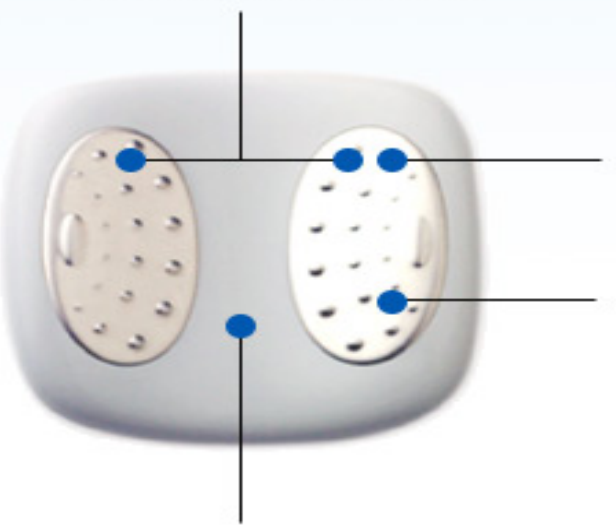
Skin Temperature

Measures the surface temperature of your body.

\section{Heat Flux}

Measures the rate at which heat is dissipating from your body.

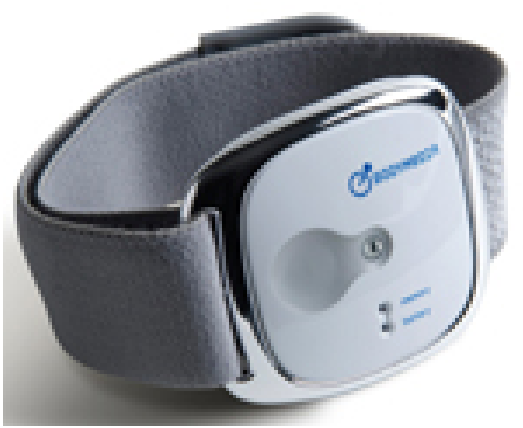


APPENDIX B

\section{COSMED K4B ${ }^{2}$}
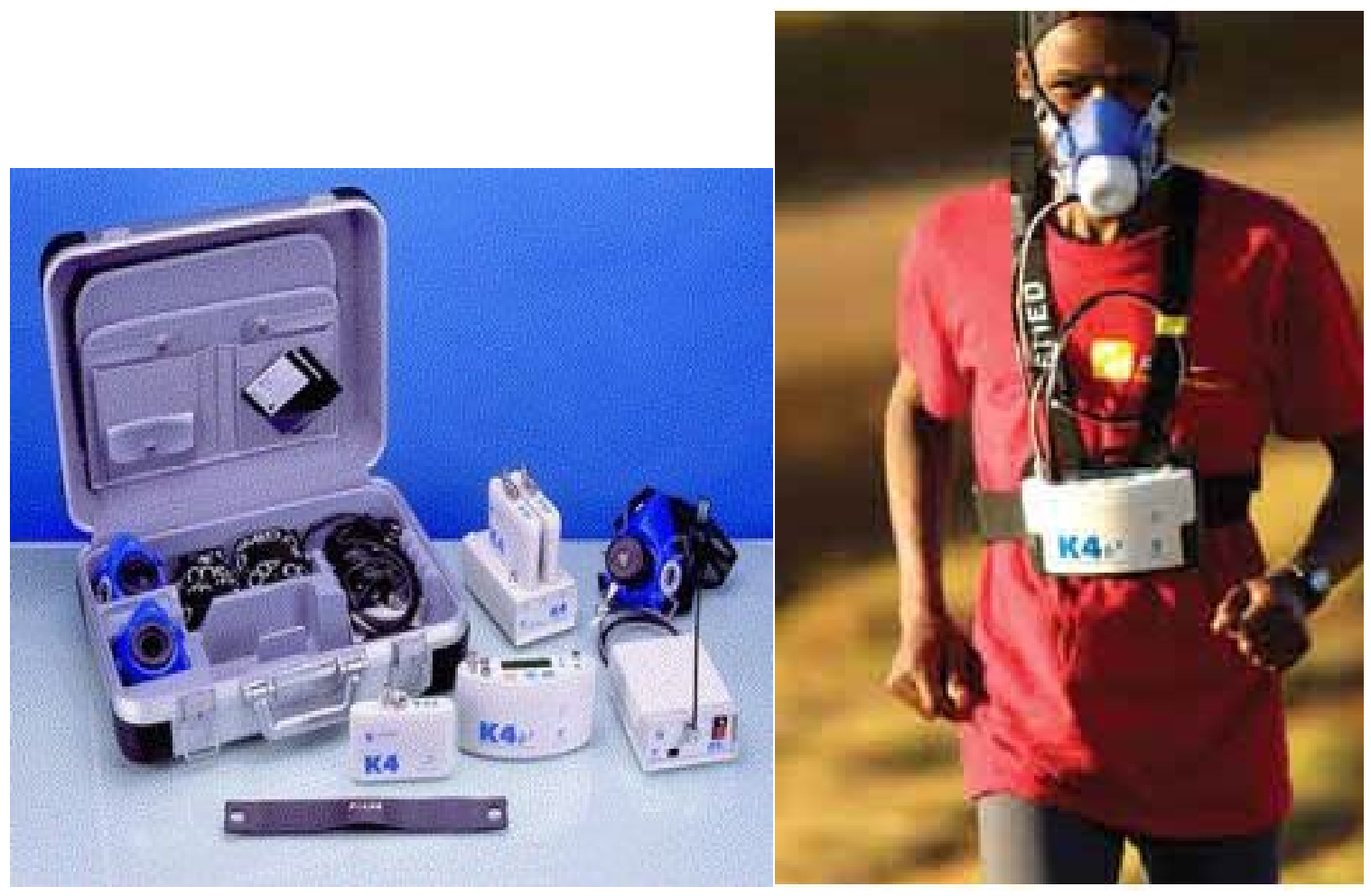
APPENDIX C

C.1 20 M SHUTTLE TEST $\mathrm{VO}_{2}$ CONVERSION

\begin{tabular}{|c|c|c|c|c|c|}
\hline Level & Shuttle & VO2 Max & Level & Shuttle & $\begin{array}{c}\text { Predicted } \\
\text { VO2 Max }\end{array}$ \\
\hline 4 & 2 & 26.8 & 5 & 2 & 30.2 \\
\hline 4 & 4 & 27.6 & 5 & 4 & 31.0 \\
\hline 4 & 6 & 28.3 & 5 & 6 & 31.8 \\
\hline 4 & 9 & 29.5 & 5 & 9 & 32.9 \\
\hline & & & & & \\
\hline Level & Shuttle & VO2 Max & Level & Shuttle & $\begin{array}{c}\text { Predicted } \\
\text { VO2 Max }\end{array}$ \\
\hline 6 & 2 & 33.6 & 7 & 2 & 37.1 \\
\hline 6 & 4 & 34.3 & 7 & 4 & 37.8 \\
\hline 6 & 6 & 35.0 & 7 & 6 & 38.5 \\
\hline 6 & 8 & 35.7 & 7 & 8 & 39.2 \\
\hline 6 & 10 & 36.4 & 7 & 10 & 39.9 \\
\hline & & & & & Predicted \\
\hline Level & Shuttle & VO2 Max & Level & Shuttle & Max \\
\hline 8 & 2 & 40.5 & 9 & 2 & 43.9 \\
\hline 8 & 4 & 41.1 & 9 & 4 & 44.5 \\
\hline 8 & 6 & 41.8 & 9 & 6 & 45.2 \\
\hline 8 & 8 & 42.4 & 9 & 8 & 45.8 \\
\hline 8 & 11 & 43.3 & 9 & 11 & 46.8 \\
\hline & & & & & \\
\hline Level & Shuttle & VO2 Max & Level & Shuttle & $\begin{array}{c}\text { Predicted } \\
\text { VO2 Max }\end{array}$ \\
\hline 10 & 2 & 47.4 & 11 & 2 & 50.8 \\
\hline 10 & 4 & 48.0 & 11 & 4 & 51.4 \\
\hline & & & & & \\
\hline
\end{tabular}




\begin{tabular}{|c|c|c|c|c|c|}
\hline 10 & 6 & 48.7 & 11 & 6 & 51.9 \\
\hline 10 & 8 & 49.3 & 11 & 8 & 52.5 \\
\hline 10 & 11 & 50.2 & 11 & 10 & 53.1 \\
\hline & & & 11 & 12 & 53.7 \\
\hline Level & Shuttle & V02 Max & Level & Shuttle & $\begin{array}{l}\text { Predicted } \\
\text { V02 Max }\end{array}$ \\
\hline 12 & 2 & 54.3 & 13 & 2 & 57.6 \\
\hline 12 & 4 & 54.8 & 13 & 4 & 58.2 \\
\hline 12 & 6 & 55.4 & 13 & 6 & 58.7 \\
\hline 12 & 8 & 56.0 & 13 & 8 & 59.3 \\
\hline 12 & 10 & 56.5 & 13 & 10 & 59.8 \\
\hline 12 & 12 & 57.1 & 13 & 13 & 60.6 \\
\hline Level & Shuttle & V02 Max & Level & Shuttle & $\begin{array}{l}\text { Predicted } \\
\text { V02 Max }\end{array}$ \\
\hline 14 & 2 & 61.1 & 15 & 2 & 64.6 \\
\hline 14 & 4 & 61.7 & 15 & 4 & 65.1 \\
\hline 14 & 6 & 62.2 & 15 & 6 & 65.6 \\
\hline 14 & 8 & 62.7 & 15 & 8 & 66.2 \\
\hline 14 & 10 & 63.2 & 15 & 10 & 66.7 \\
\hline 14 & 13 & 64.0 & 15 & 13 & 67.5 \\
\hline Level & Shuttle & VO2 Max & Level & Shuttle & $\begin{array}{l}\text { Predicted } \\
\text { V02 Max }\end{array}$ \\
\hline 16 & 2 & 68.0 & 17 & 2 & 71.4 \\
\hline 16 & 4 & 68.5 & 17 & 4 & 71.9 \\
\hline 16 & 6 & 69.0 & 17 & 6 & 72.4 \\
\hline 16 & 8 & 69.5 & 17 & 8 & 72.9 \\
\hline 16 & 10 & 69.9 & 17 & 10 & 73.4 \\
\hline 16 & 12 & 70.5 & 17 & 12 & 73.9 \\
\hline 16 & 14 & 70.9 & 17 & 14 & 74.4 \\
\hline Level & Shuttle & V02 Max & Level & Shuttle & $\begin{array}{l}\text { Predicted } \\
\text { V02 Max }\end{array}$ \\
\hline 18 & 2 & 74.8 & 19 & 2 & 78.3 \\
\hline 18 & 4 & 75.3 & 19 & 4 & 78.8 \\
\hline 18 & 6 & 75.8 & 19 & 6 & 79.2 \\
\hline 18 & 8 & 76.2 & 19 & 8 & 79.7 \\
\hline
\end{tabular}




\begin{tabular}{|c|c|c|c|c|c|}
\hline 18 & 10 & 76.7 & 19 & 10 & 80.2 \\
\hline 18 & 12 & 77.2 & 19 & 12 & 80.6 \\
\hline 18 & 15 & 77.9 & 19 & 15 & 81.3 \\
\hline Level & Shuttle & V02 Max & Level & Shuttle & $\begin{array}{l}\text { Predicted } \\
\text { VO2 Max }\end{array}$ \\
\hline 20 & 2 & 81.8 & 21 & 2 & 85.2 \\
\hline 20 & 4 & 82.2 & 21 & 4 & 85.6 \\
\hline 20 & 6 & 82.6 & 21 & 6 & 86.1 \\
\hline 20 & 8 & 83.0 & 21 & 8 & 86.5 \\
\hline 20 & 10 & 83.5 & 21 & 10 & 86.9 \\
\hline 20 & 12 & 83.9 & 21 & 12 & 87.4 \\
\hline 20 & 14 & 84.3 & 21 & 14 & 87.8 \\
\hline 20 & 16 & 84.8 & 21 & 16 & 88.2 \\
\hline
\end{tabular}




\section{C.2 20 M SHUTTLE TEST}

\begin{tabular}{|l|l|l|l|l|l|l|l|}
\hline Level & Shuttles & $\begin{array}{c}\text { Speed } \\
\mathbf{( k m / h )}\end{array}$ & $\begin{array}{c}\text { Shuttle } \\
\text { Time } \\
\text { (seconds) }\end{array}$ & $\begin{array}{c}\text { Total } \\
\text { level } \\
\text { time } \mathbf{( s )}\end{array}$ & $\begin{array}{c}\text { Distance } \\
\mathbf{( m )}\end{array}$ & $\begin{array}{c}\text { Cumulative } \\
\text { Distance } \\
\mathbf{( m )}\end{array}$ & $\begin{array}{c}\text { Cumulative } \\
\text { Time } \\
\text { (min and } \\
\text { seconds) }\end{array}$ \\
\hline 1 & 7 & 8.0 & 9.00 & 63.00 & 140 & 140 & $1: 03$ \\
\hline 2 & 8 & 9.0 & 8.00 & 64.00 & 160 & 300 & $2: 07$ \\
\hline 3 & 8 & 9.5 & 7.58 & 60.63 & 160 & 460 & $3: 08$ \\
\hline 4 & 9 & 10.0 & 7.20 & 64.80 & 180 & 640 & $4: 12$ \\
\hline 5 & 9 & 10.5 & 6.86 & 61.71 & 180 & 820 & $5: 14$ \\
\hline 6 & 10 & 11.0 & 6.55 & 65.50 & 200 & 1020 & $6: 20$ \\
\hline 7 & 10 & 11.5 & 6.26 & 62.61 & 200 & 1220 & $7: 22$ \\
\hline 8 & 11 & 12.0 & 6.00 & 66.00 & 220 & 1440 & $8: 28$ \\
\hline 9 & 11 & 12.5 & 5.76 & 63.36 & 220 & 1660 & $9: 31$ \\
\hline 10 & 11 & 13.0 & 5.54 & 60.92 & 220 & 1880 & $10: 32$ \\
\hline 11 & 12 & 13.5 & 5.33 & 64.00 & 240 & 2120 & $11: 36$ \\
\hline 12 & 12 & 14.0 & 5.14 & 61.71 & 240 & 2360 & $12: 38$ \\
\hline 13 & 13 & 14.5 & 4.97 & 64.55 & 260 & 2620 & $13: 43$ \\
\hline 14 & 13 & 15.0 & 4.80 & 62.40 & 260 & 2880 & $14: 45$ \\
\hline 15 & 13 & 15.5 & 4.65 & 60.39 & 260 & 3140 & $15: 46$ \\
\hline 16 & 14 & 16.0 & 4.50 & 63.00 & 280 & 3420 & $16: 49$ \\
\hline 17 & 14 & 16.5 & 4.36 & 61.09 & 280 & 3700 & $17: 50$ \\
\hline 18 & 15 & 17.0 & 4.24 & 63.53 & 300 & 4000 & $18: 54$ \\
\hline 19 & 15 & 17.5 & 4.11 & 61.71 & 300 & 4300 & $19: 56$ \\
\hline 20 & 16 & 18.0 & 4.00 & 64.00 & 320 & 4620 & $21: 00$ \\
\hline 21 & 16 & 18.5 & 3.89 & 62.27 & 320 & 4940 & $22: 03$ \\
\hline & & & & & & & \\
\hline
\end{tabular}




\title{
APPENDIX D
}

\section{[DYNAMIC WARM-UP]}

All exercises will be performed for $1 / 2$ the length of the basketball court ( $47 \mathrm{ft}$.) down and back.

\author{
Jog \\ High Knees \\ Butt-kicks \\ Walking Lunges \\ Lateral Lunges \\ Knee Hugs \\ Tin man \\ Shuffle \\ Carioca \\ Power Skip
}

Running- 50\%/75\%/100\% (Full court 94 ft.) 
APPENDIX E

\section{0-MINUTE BASKETBALL WORKOUT PROTOCOL}

Progressive Defensive Slide Drill- 1 @ 50\%, 2 @ 75\%, 2 @ 100\%

1-minute transition

Mikan Drill- 2 x 45 second w/ 15-second rest

1-minute transition

1/2 Court Speed Lay-Up Drill- 2 x 45 second w/ 15-second rest

1-minute transition

Victories- 2 x 36 seconds w/ 24-second rest

1-minute transition

Free Throw- 5

Water break- 3-minute rest

Medicine Ball Plyometric- 2 x 45 second w/ 15-second rest

1-minute transition

$1 / 2$ Court Dribbling Drill- each drill $x 1$ up and back

- Right hand up/ Left hand back

- Crossover (zigzag)

- Between the legs (zigzag)

- Behind the back (zigzag)

- Spin dribble (zigzag)

1-minute transition

Toss Out Shooting Drill- 5 spot shooting around the horn 2 x 45 second w/ 15-second rest 1-minute transition

Free Throw- 5 


\section{E.1 PROGRESSIVE DEFENSIVE SLIDES (SIGMON, 2003)}

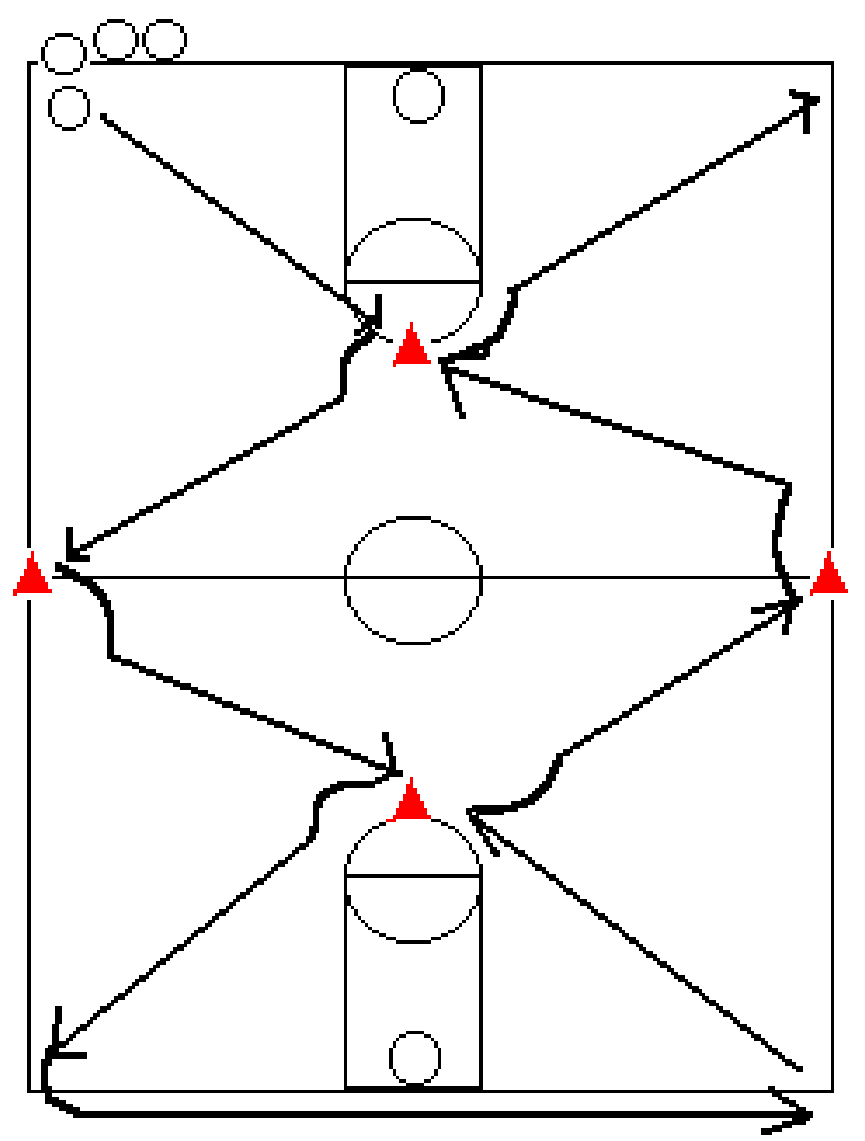




\section{E.2 MIKAN DRILL (SIGMON, 2003)}
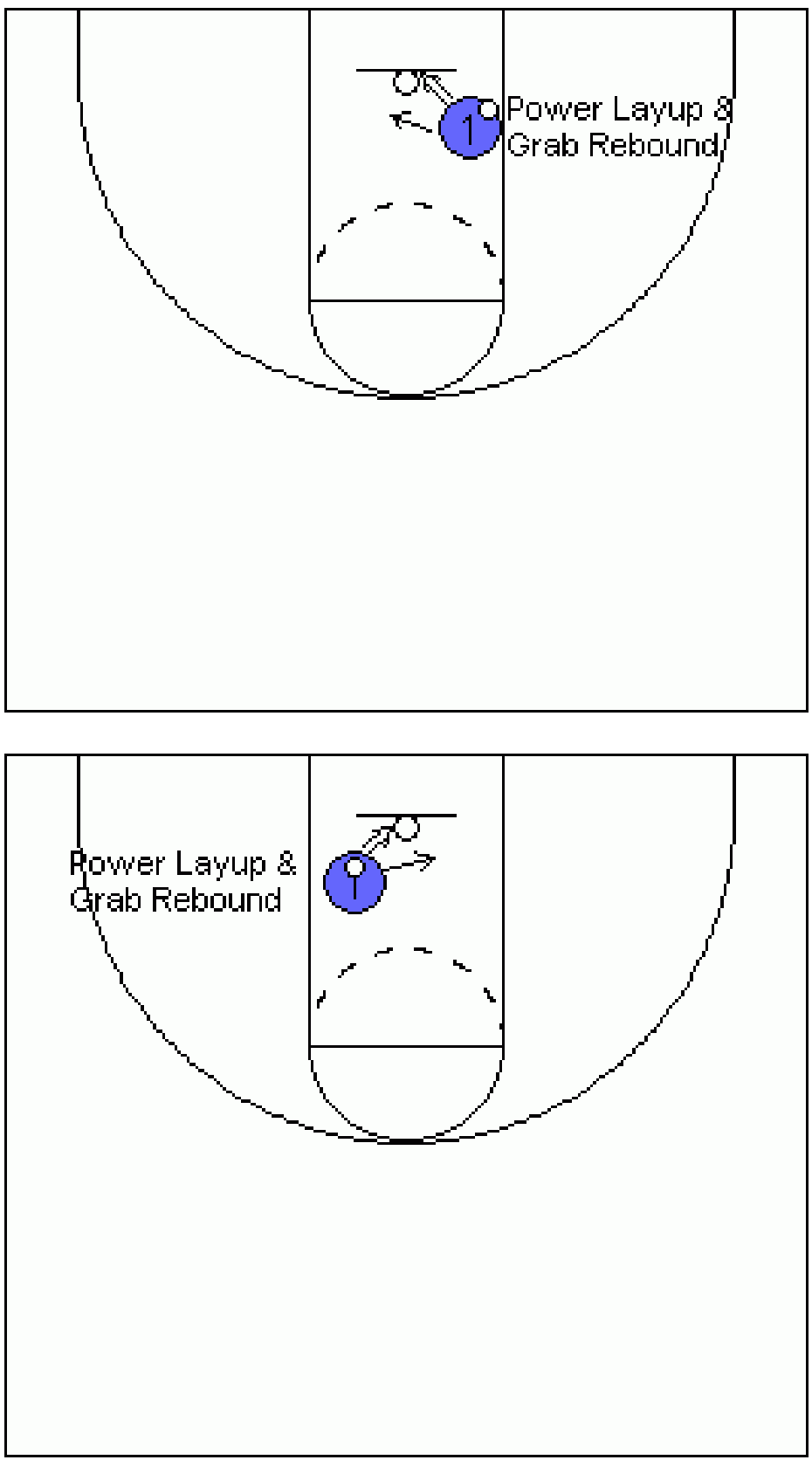


\section{E.3 $1 / 2$ COURT SPEED LAY-UP DRILL (SIGMON, 2003)}

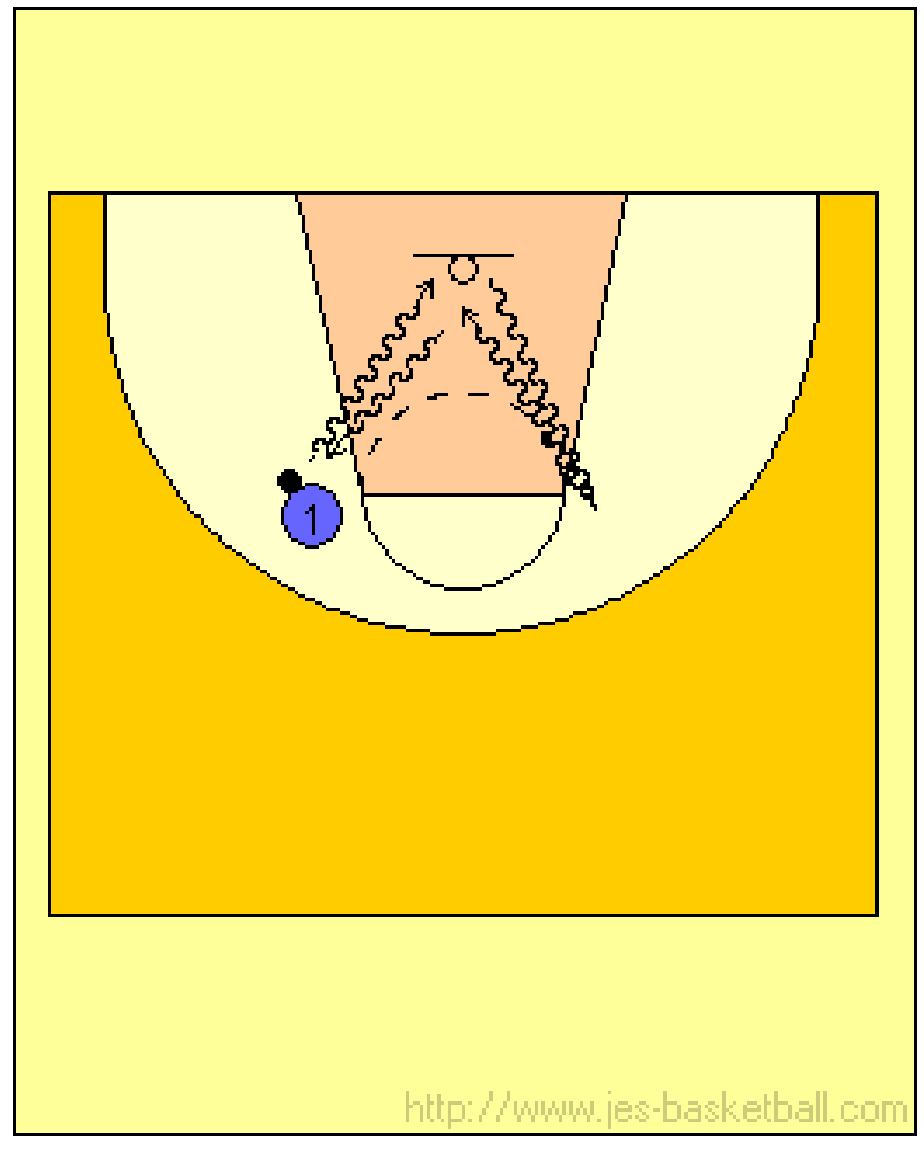




\section{E.4 SUICIDE DRILL (SIGMON, 2003)}

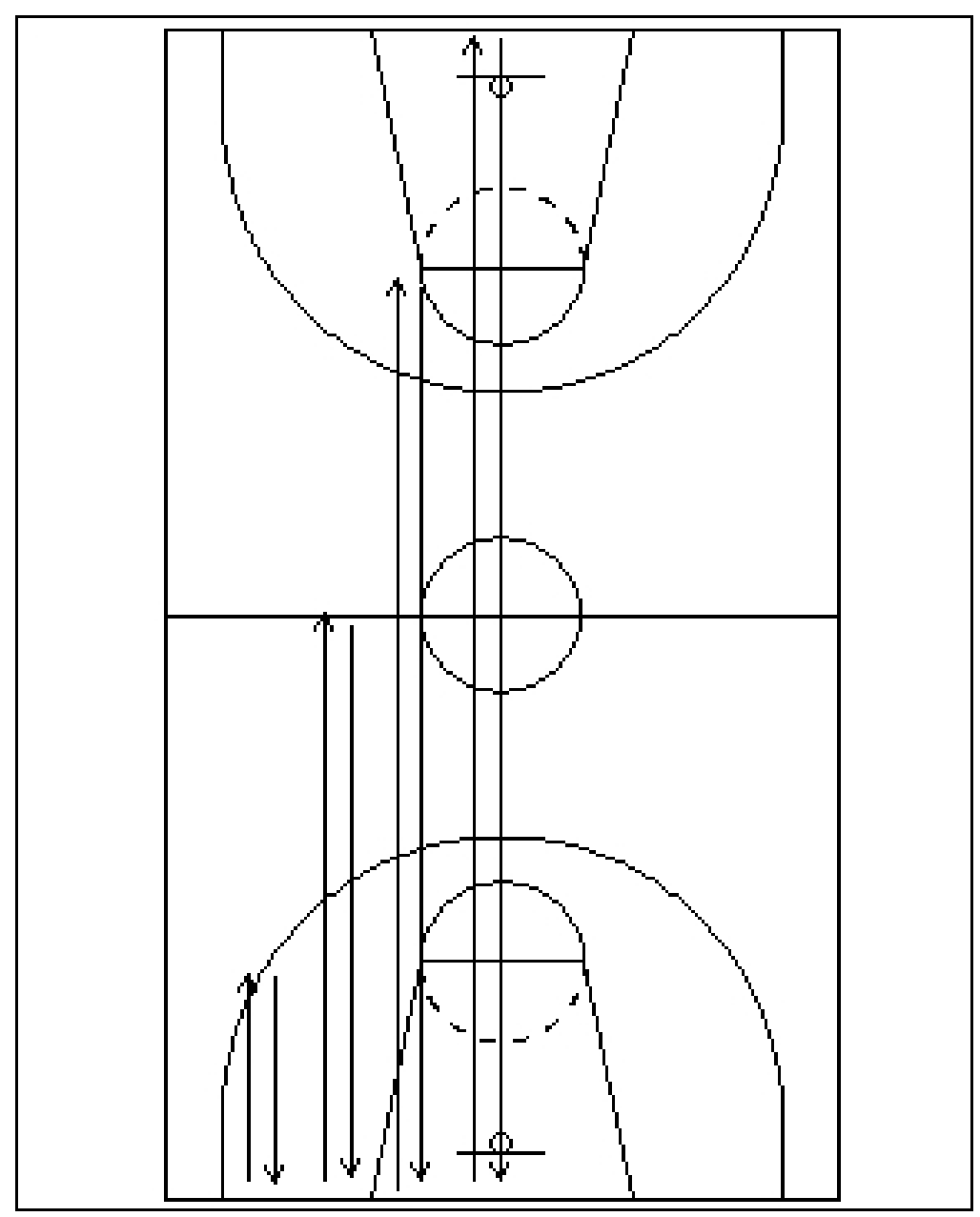




\section{E.5 FREE THROW (SIGMON, 2003)}

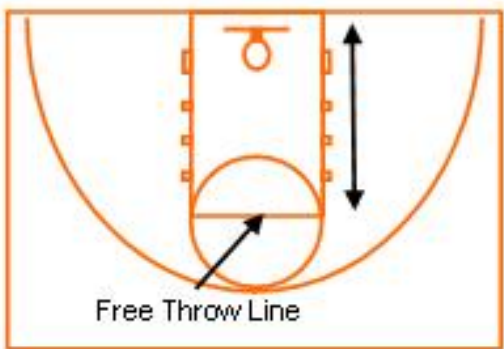

The distance from the Back board to Free Throw Line is 15 feet!

E.6 MEDICINE BALL PLYOMETRICS (SIGMON, 2003)

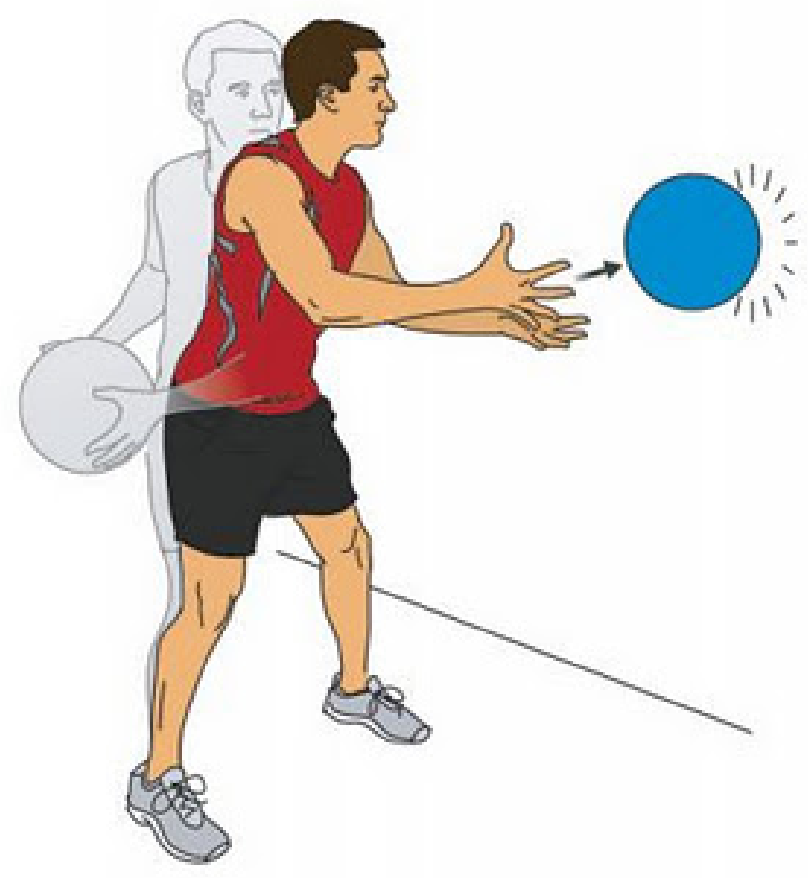


E.7 $1 / 2$ COURT DRIBBLING DRILL (SIGMON, 2003)

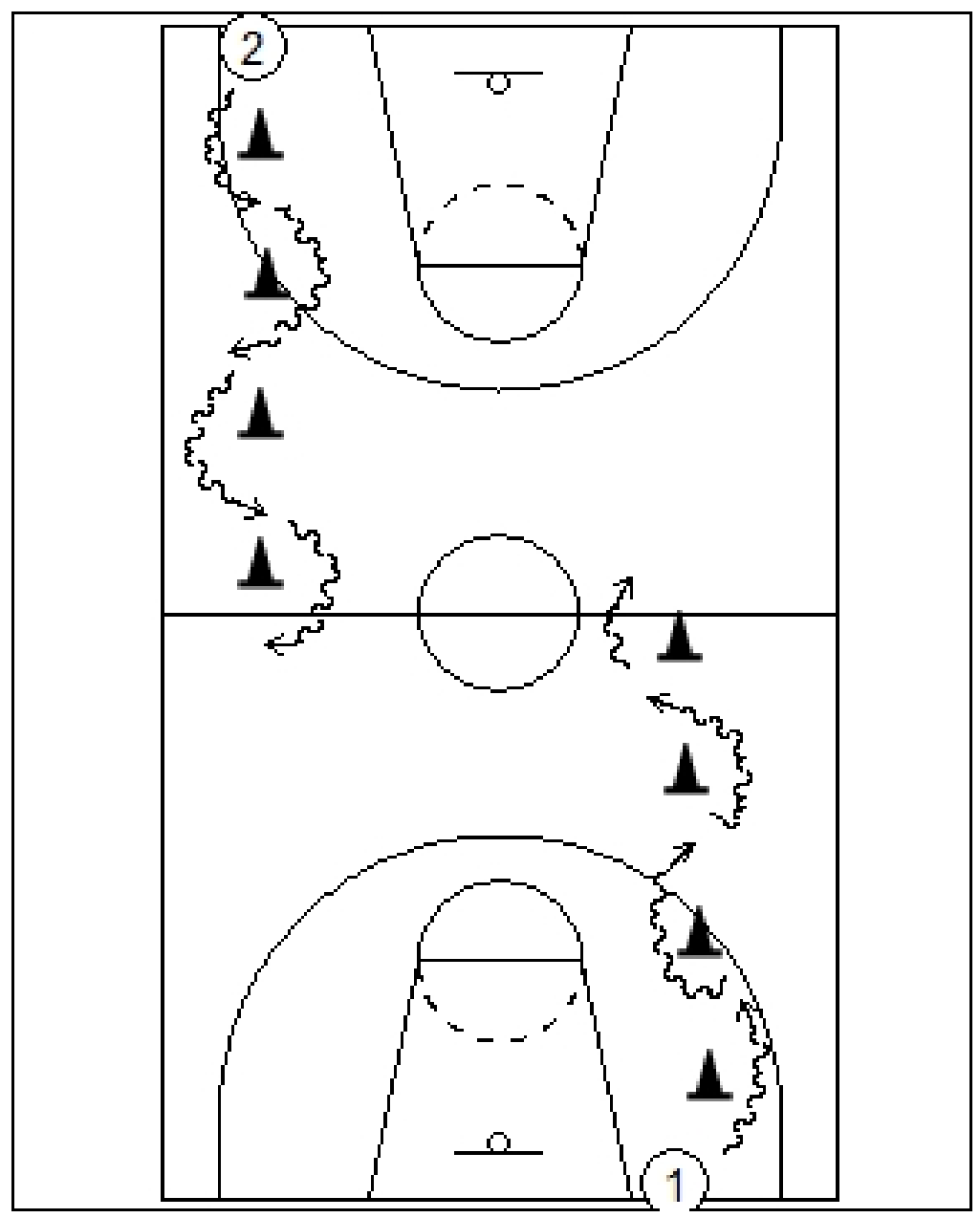


E.8 TOSS OUT SHOOTING DRILL (SIGMON, 2003)

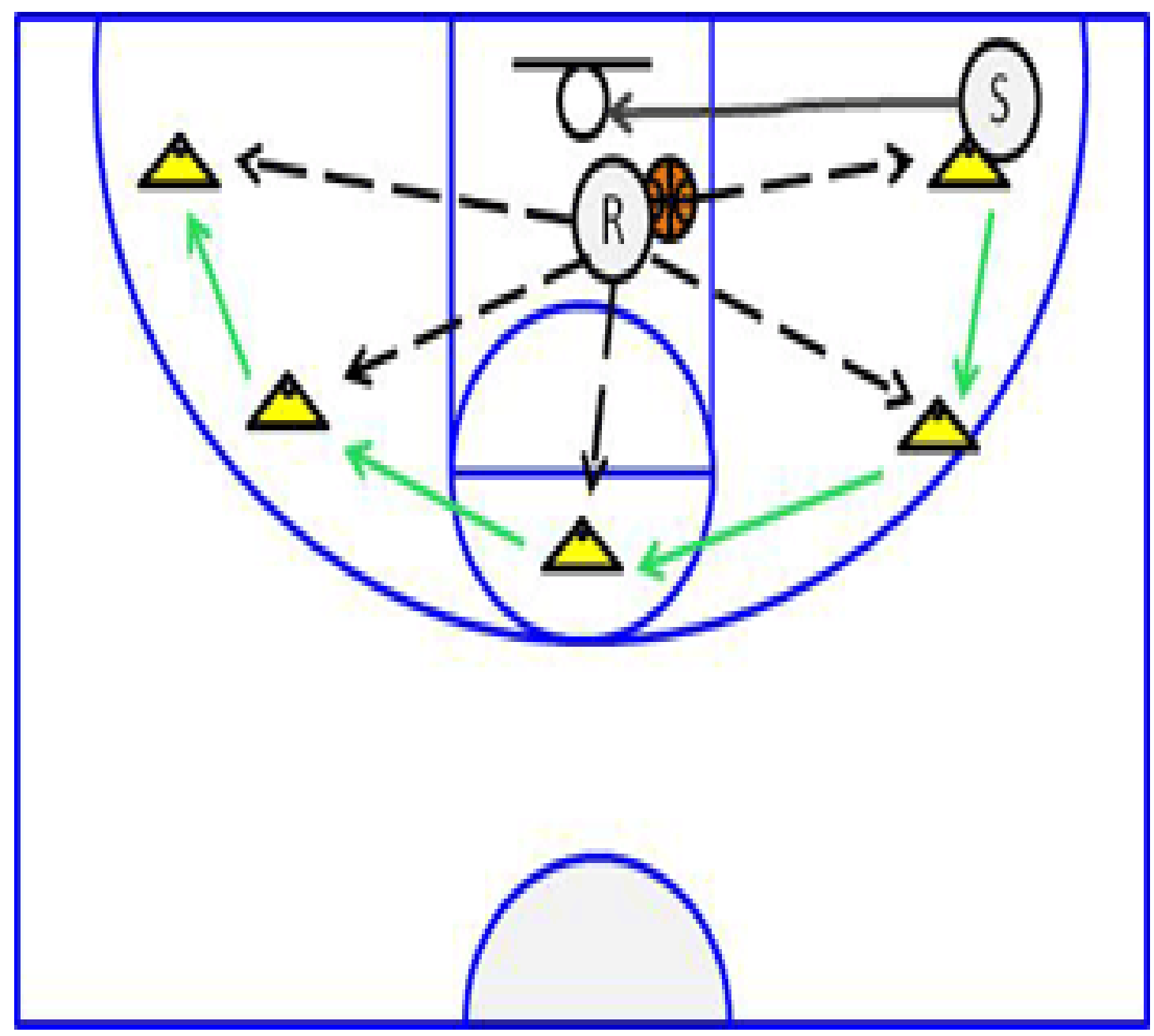




\section{APPENDIX F}

\section{OMNI RATINGS OF PERCEIVED EXERTION}

\section{ORIGINIAL ADULT OMNI-WALK/RUN SCALE INSTRUCTIONS}

\section{Definition of RPE:}

"The perception of physical exertion is defined as the subjective intensity of effort, strain, discomfort, and/or fatigue that you feel during exercise".

" Please use the numbers on this scale to tell us how your body feels when you are walking/running. Please look at the person at the bottom of the hill who is just starting to walk/run (point to person on chart). If you feel like this person when you are walking/running, the exertion will be EXTREMELY EASY. In this case, your rating should be a zero. Now look at the person who is exhausted at the top of the hill (point to the person on the chart). If you feel like this person when walking/running, the exertion will be EXTEMELY HARD. In this case, your rating should be a 10. If you feel somewhere between Extremely Easy (0) and Extremely Hard (10) then give a number between 0 and 10.

We will ask you to point to a number that tells how your whole body, chest, and legs feel. Remember, there are no right or wrong answers. Use both the pictures and words to help you select a number. Use any of the numbers to tell us how you feel when walking/running.

\section{Trial I only}

-We will ask you to give us an RPE at the end of each drill and an overall session RPE 5 minutes post session.

Trial II only

- We will ask you to give us an RPE Immediately following the session along with an overall session RPE 5 minutes post session. 


\section{F.1 F.1 OMNI WALK/RUN SCALE}

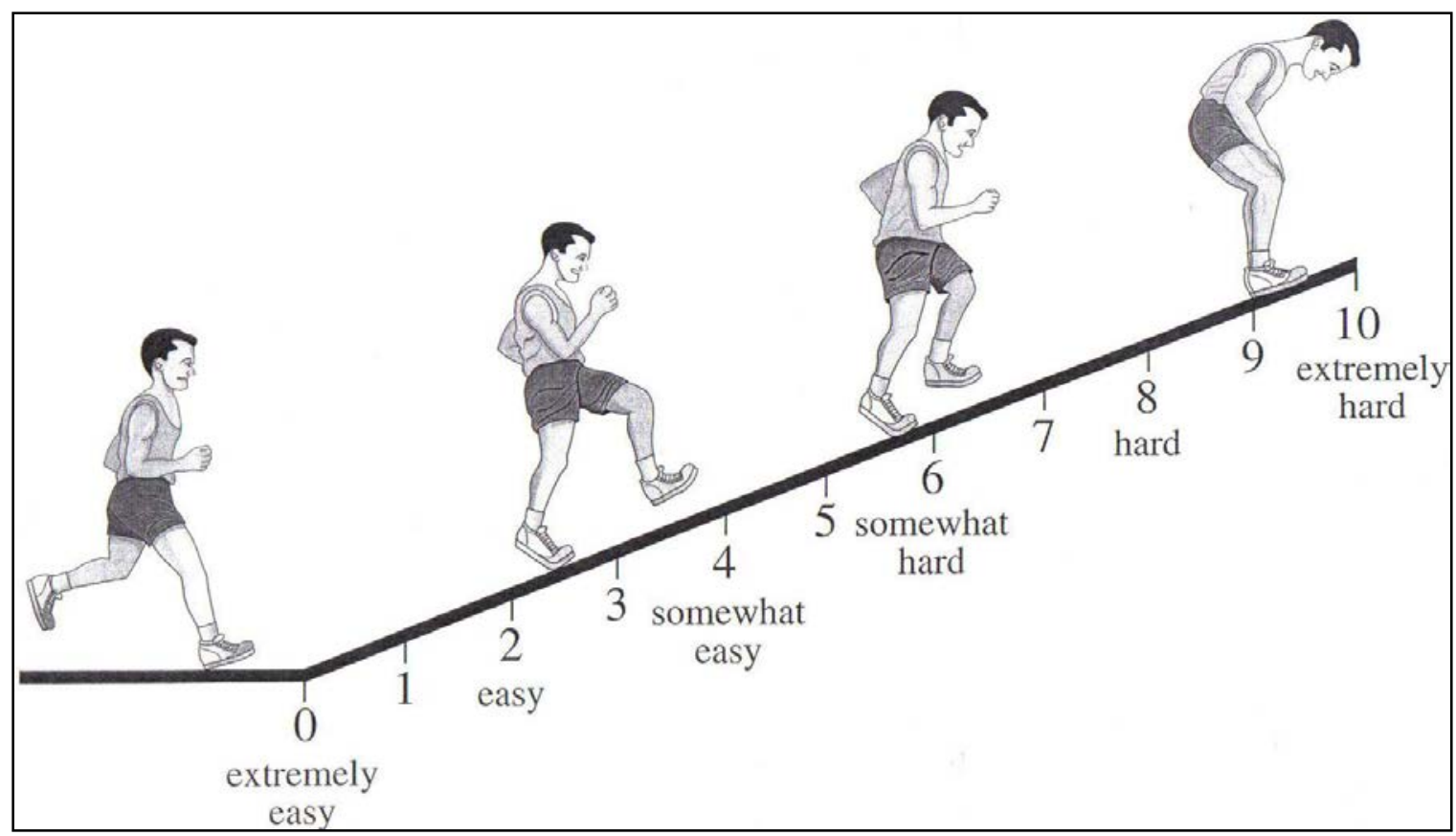




\section{APPENDIX G}

ID \#

\section{University of Pittsburgh}

\section{Center for Exercise and Health-Fitness Research}

\section{MEDICAL HISTORY}

\section{YES}

1. History of heart problems, chest pain, or stroke?

2. Have you ever been diagnosed with MI or Peripheral Vascular Disease?

3. Increased blood pressure?

4. Any chronic illness or condition?

5. Difficulty with physical exercise?

6. Advice from a physician not to exercise?

7. Recent surgery? (Last 12 months)

8. Pregnancy? (Now or within the last 3 months)

9. History of breathing or lung problems?

10. Muscle, joint, back disorder, or any previous injury still affecting you?

11. Diabetes or thyroid conditions?

12. Cigarette smoking habit?

13. Increased blood cholesterol?

14. History of heart problems in your immediate family?

15. Do you have any implantable devices (i.e. pacemaker, defibrillator)

16. Hernia or any condition that may be aggravated by lifting weights?

17. Do you have any condition limiting your movement?

18. Are you aware of being allergic to any drugs or insect bites?

19. Do you have asthma?

20. Do you have epilepsy, convulsions, or seizures of any kind?

21. Do you follow any specific diet?

Please explain in detail any "YES" answers:

\section{Family History}

Has any member of you family had any of those listed above? 


\section{APPENDIX H}

ID \#

\section{University of Pittsburgh \\ Center for Exercise and Health-Fitness Research \\ Physical Activity Readiness Questionnaire (PAR-Q)}

1. Has your doctor ever said that you have a heart condition and that you should only do physical activity recommended by a doctor?

No __ Yes __ If yes, specify:

2. Do you feel pain in your chest when you do physical activity?

No __ Yes __ If yes, specify:

3. In the past month, have you had chest pain when you were not doing physical activity?

No __ Yes __ If yes, specify:

4. Do you lose your balance because of dizziness or do you ever lose consciousness?

No __ Yes __ If yes, specify:

5. Do you have a bone or joint problem that could be made worse by a change in your physical activity?

$$
\text { No __ Yes __ If yes, specify: }
$$

6. Is your doctor currently prescribing drugs (for example, water pills) for a blood pressure or heart condition?

$$
\text { No __ Yes __ If yes, specify: }
$$

7. Do you know of any other reason why you should not do physical activity?

No __ Yes __ If yes, specify: 


\section{APPENDIX I}

\section{COOL-DOWN}

Walk 2 times up and back the length of the court.

Standing Shoulder Stretch- 20 second hold for each arm

Standing Triceps Stretch-20 second hold for each arm

Standing Calf Stretch-20 second hold

Sitting Hamstring Stretch-20 second hold

Sitting Single Leg Hamstring Stretch-20 second hold each leg

Sitting Inner Thigh Stretch (butterfly)- 20-second hold

Lying Quadriceps Stretch- 20 second hold each leg

Sitting Hip Flexor Stretch- 20 second hold each leg

Sitting Back Stretch- 20 second hold each side 


\section{APPENDIX J}

\section{INFORMED CONSENT}

\section{University of Pittsburgh}

School of Education

Health, Physical and Recreation Education

Center for Exercise and Health-Fitness Research
A149B Trees Hall

Pittsburgh, Pennsylvania 15261

412-648-8251

Fax: $412-648-7092$

\section{INFORMED CONSENT}

TITLE: VALIDATION OF THE BODYMEDIA MINI ARMAND TO ESTIMATE

ENERGY EXPENDITURE OF FEMALE BASKETBALL PLAYERS DURING VARIABLE

INTENSITY GAME-LIKE CONDITIONS

PRINCIPAL INVESTIGATOR: Monica Peterson Taylor, M.S.

Graduate Student Assistant, Center for Exercise and

Health-Fitness Research

A149B Trees Hall

Phone: (412) 648-8251 Fax: (412) 648-7092

Email:mop15@pitt.edu

Department of Health and Physical Activity

School of Education 


\section{CO-INVESTIGATORS:}

Elizabeth F. Nagle, Ph.D.

Assistant Professor and Assistant Director, Center for Exercise and Health-Fitness Research

149 Trees Hall

Phone: (412) 648-8265 Fax: (412) 648-7092

Email: nagle@pitt.edu

Department of Health and Physical Activity

School of Education

\section{SOURCE OF SUPPORT: Council for Graduate Students in Education}

\section{Why is this research being done?}

The purpose of this study is to test the ability of a small monitoring device to assess energy expenditure in female basketball players. Basketball is a high intensity sport that requires speed, quickness, and agility. There are also high-energy requirements for work at many different intensities. Which is why there is a need for an accurate portable method to quantify energy expenditure in basketball player. If the Mini Armband can provide an accurate measure of energy expenditure when assessing collegiate women's basketball players the information can be used for many purposes, such as providing insight into the metabolic demands of specific workouts, improving practice/workout design, improving the assessment of recovery needs, and decreasing the potential for overtraining.

\section{Who is being asked to take part in this research study?}

Women's basketball players (18-30 yrs old) will participate as subjects in this investigation. Participation will last approximately 2 weeks. You are being invited to take part in this research study because you are healthy and participate in women's basketball. To minimize risks associated with maximal aerobic exercise testing, you will be asked to complete a Physical Activity Readiness Questionnaire (PAR-Q) and a medical history form which asks questions about your current health status. You will not be eligible to participate in this research study if you have muscle or bone disease, heart disease, have had a prior heart attack, blockages in legs, lung disease, high/low blood sugar and/or if you are knowingly pregnant.

\section{What procedures will be performed for research purposes?}

If you decide to take part in this research study, you will be required to complete two separate visits to the laboratory. The two visits will involve 10 - 30 minute exercise tests, each separated by a 7-day period. Both Trial II and Trial II will include exercise on a basketball court.

If an abnormal response occurs during exercise, such as chest pain, the test will be immediately stopped and you will be given proper medical attention. Emergency equipment will be on site for all testing procedures and research staff is certified in CPR and First Aid by the American Red Cross. If you have an abnormal response to the cycle test, you will be told of the findings and will be encouraged to contact your primary care clinician. 
All procedures will take place in the Human Energy Research Laboratory at the Center for Exercise and Health-Fitness Research and the gymnasium located in Trees Hall at the University of Pittsburgh. All testing sessions will be administered by trained staff members from the Center for Exercise and Health-Fitness Research.

\section{Pre-Testing Procedures:}

- Before starting the study protocol, you will complete a medical history form and a physical activity readiness questionnaire (PAR-Q) to determine risk. These forms will take less than ten minutes to complete.

- During both exercise trials, a heart rate monitor will be positioned around your chest and secured in place with an elastic strap. The Mini Armband will be positioned on your left arm. A rubber facemask will be attached to your face, covering your nose and mouth during exercise to determine the amount of oxygen that you use during exercise. Some individuals become anxious when fitted with the mask. If this occurs to you, please inform the individual performing the test and the test will be stopped and mask removed. Your heart rate and the amount of oxygen that your body uses will be measured during exercise.

- Prior to all of the exercise trials, you will receive standard instructions for use of the rate of perceived exertion (RPE) scale. The investigator will first read to you the definitions of RPE. A set of instructions on how to use the corresponding scale during the exercise trials will then be read to you.

- Prior to each exercise session you will be asked to abstain from caffeine intake and eating for four hours, to reduce any thermic effects of food on energy expenditure. You will also be asked to not participate in any vigorous exercise activities (any activity that takes your heart rate above $85 \%$ of max) for at least 12 hours prior to testing.

\section{Trial I: 20-meter Shuttle Run Test}

- Your body height and weight will be measured using a standard physicians’ scale.

- Body composition will be assessed using a Tanita bioelectrical impedance analyzer (BIA). The BIA is a non-invasive pain-free procedure for assessing your body fat and muscle. The BIA instrument transmits a low-level electrical impulse through the body. You will remove your shoes and socks and stand on the Tanita BIA scale for approximately 10 seconds to obtain the body composition measurement. During the body composition measurement there may be a potential for the hair on your arms and legs to stand up. 
- The Mini Armband will be positioned on your upper left arm. The Cosmed K4b ${ }^{2}$ unit will also be fitted into an over the shoulder harness and placed on your back this will feel like a backpack.

- You will be escorted to the gymnasium where you will sit for 15 minutes in a resting position.

- Following the resting period, you will engage in a standardized dynamic warm-up protocol led by primary investigator.

- You will be asked to perform a 20-meter shuttle running test. The exercise protocol will begin at low intensity. The intensity will increase every minute. You will be encouraged to continue until completely fatigued. However, you may stop the test at any time for any reason.

- The test will last 9-13 minutes and you will go until you are completely fatigued

- After completion of the 20-meter shuttle running test you will go through a cool-down and static stretching lead by the primary investigator.

\section{Trial II: 30-Minute Basketball Skills Session}

- Your body height and weight will be measured using a standard physicians’ scale.

- Body composition will be assessed using a Tanita bioelectrical impedance analyzer (BIA). The BIA is a non-invasive pain-free procedure for assessing your body fat and muscle. The BIA instrument transmits a low-level electrical impulse through the body. You will remove your shoes and socks and stand on the Tanita BIA scale for approximately 10 seconds to obtain the body composition measurement. During the body composition measurement there may be a potential for the hair on your arms and legs to stand up.

- The Mini Armband will be positioned on your upper left arm. The Cosmed K4b ${ }^{2}$ unit will also be fitted into an over the shoulder harness and placed on your back this will feel like a backpack.

- You will be escorted to the gymnasium where you will sit for 15 minutes in a resting position.

- Following the resting period, you will engage in a standardized dynamic warm-up protocol led by primary investigator.

- You will be asked to perform a 30-minute individual basketball training session. The protocol will involve 8 different basketball drills which include defensive slides, lay-ups, free throw shooting, dribbling, and mid range shooting. Each drill will be a designated length, and the entire skills session will last no longer than 30 minutes. 
- After completion of the 30-minute basketball skills session you will go through a cooldown and static stretching lead by the primary investigator.

What are the possible risks, side effects, and discomforts of this research study?

\section{Risks of the Exercise Test}

Abnormal responses, such as excessive rises in blood pressure, mental confusion, shortness of breath, chest pain, heart attack, and death, to maximal aerobic exercise tests in young healthy adults are rare, occurring in less than $1 \%$ of people (less than 1 out of 100 people tested). However, some common risks, occurring in 1\% to $25 \%$ of people (1 to 25 out of 100 people tested), of maximal exercise testing include; heavy breathing, dizziness, muscle fatigue, headache, and overall fatigue.

\section{Risks of the Study Monitors}

Risks associated with study monitors (e.g. heart rate monitor and face mask) include redness, irritation, and chafing. Similar to a sports-bra or other exercise wear which provides secure support and contact with the ribcage, subjects who wear a Polar monitor may encounter some infrequent chafing that will dissipate upon removal.

\section{Risk of Breach of Confidentiality}

There is a potential risk due to the group format of the orientation session. Teammates may know of your participation in the study. However, personal information will not be shared and any information about you obtained from this research will be kept as confidential (private) as possible. Your identity on these records will be indicated by a case number rather than by your name, and the information linking these case numbers with your identity will be kept separate from the research records.

\section{What are possible benefits from taking part in this study?}

You will likely receive no direct benefit from taking part in this research study. However, you will receive information regarding your aerobic fitness level and percent body fat.

If I agree to take part in this research study, will I be told of any new risks that may be found during the course of the study? 
You will be promptly notified if, during the conduct of this research study, any new information develops which may cause you to change your mind about continuing to participate.

\section{Will my insurance provider or I be charged for the costs of any procedures performed as part of this research study?}

Neither you, nor your insurance provider, will be charged for the costs of any procedures performed for the purpose of this research study.

\section{Will I be paid if I take part in this research study?}

You will not be paid for your participation in this study.

\section{Who will pay if I am injured as a result of taking part in this study?}

University of Pittsburgh researchers and their associates who provide services at UPMC recognize the importance of your voluntary participation in their research studies. These individuals and their staffs will make reasonable efforts to minimize, control, and treat any injuries that may arise as a result of this research. If you believe that you are injured as a result of the research procedures being performed, please immediately contact the Principal Investigator or one of the Co-Investigators listed on the first page of this form.

Emergency medical treatment for injuries solely and directly related to your participation in this research study will be provided to you by the hospitals of the UMPC.

It is possible that the UPMC may bill your insurance provider for the costs of this emergency treatment, but none of these costs will be charged directly to you. If your researchrelated injury requires medical care beyond this emergency treatment, you will be responsible for the cost of this follow-up unless otherwise specifically stated below. There is no plan for monetary compensation. You do not, however, waive any legal rights by signing this form.

\section{Who will know about my participation in this research study?}

Any information about you obtained from this research will be kept as confidential (private) as possible. All records related to your involvement in this research study will be stored in a locked file cabinet. Your identity on these records will be indicated by a case number rather than by your name, and the information linking these case numbers with your identity will be kept separate from the research records. You will not be identified by name in any publication of the research results unless you sign a separate consent form giving your permission (release). 


\section{Will this research study involve the use or disclosure of my identifiable medical information?}

This research study will not involve the use or disclosure of any identifiable medical information.

\section{Who will have access to identifiable information related to my participation in this research study?}

In addition to the investigators listed on the first page of this authorization (consent) form and their research staff, the following individuals will or may have access to identifiable information related to your participation in this research study:

- Authorized representatives of the University of Pittsburgh Research Conduct and Compliance Office may review your identifiable research information for the purpose of monitoring the appropriate conduct of this research study.

- In unusual cases, the investigators may be required to release identifiable information related to your participation in this research study in response to an order from a court of law. If the investigators learn that you or someone with whom you are involved is in serious danger or potential harm, they will need to inform, as required by Pennsylvania law, the appropriate agencies.

For how long will the investigators be permitted to use and disclose identifiable information related to my participation in this research study?

The investigators may continue to use and disclose, for the purposes described above, identifiable information related to your participation in this research study for a minimum of seven years after final reporting or publication of a project.

\section{Is my participation in this research study voluntary?}

Your participation in this research study, to include the use and disclosure of your identifiable information for the purposes described above, is completely voluntary. Whether or not you provide your consent for participation in this research study will have no affect on your current or future relationship with the University of Pittsburgh. Whether or not you provide your consent for participation in this research study will have no effect on your current or future medical care at a UPMC hospital or affiliated health care provider or your current or future relationship with a health care insurance provider. The decision to participate in the study will have no bearing on eligibility status, nor will it interfere with practice time.

May I withdraw, at a future date, my consent for participation in this research study?

You may withdraw, at any time, your consent for participation in this research study, to include the use and disclosure of your identifiable information for the purposes described above. Any identifiable research information recorded for, or resulting from, your participation in this 
research study prior to the date that you formally withdrew your consent may continue to be used and disclosed by the investigators for the purposes described above.

To formally withdraw your consent for participation in this research study you should provide a written and dated notice of this decision to the principal investigator of this research study at the address listed on the first page of this form.

Your decision to withdraw your consent for participation in this research study will have no effect on your current or future relationship with the University of Pittsburgh. Your decision to withdraw your consent for participation in this research study will have no effect on your current of future medical care at a UPMC hospital or affiliated health care provider or your current or your future relationship with a health care insurance provider.

\section{If I agree to take part in this research study, can I be removed from the study without my consent?}

It is possible that you may be removed from the research study by the researchers to protect your safety or if you are unable or unwilling to complete the research protocol.

\section{VOLUNTARY CONSENT}

All of the above has been explained to me and all of my questions have been answered. I understand that a copy of this consent form will be given to me and any future questions I have about this research study during the course of this study, and that such future questions will be answered by the investigators listed on the first page of this consent document at the telephone numbers given. Any questions I have about my rights as a research subject will be answered by the Human Subject Protection Advocate of the IRB Office, University of Pittsburgh (1-866-2122668). By signing this form, I agree to participate in this research study.

Participant's Name (Print)

Participant's Signature

Date 


\section{CERTIFICATION OF INFORMED CONSENT}

I certify that I have explained the nature and purpose of this research study to the abovenamed individual, and I have discussed the potential benefits, and possible risks associated with participation. Any questions, concerns or complaints the individual has about this study have been answered, and we will always be available to address future questions as they arise. I further certify that no research component of this protocol was begun until after this consent form was signed.

Printed Name of Person Obtaining Consent

Role in Research Study 


\section{APPENDIX K}

\section{0-METER SHUTTLE RUN TEST ANOVA OUTPUT}

\begin{tabular}{|l|c|c|c|c|c|}
\hline Source & Type III & df & Mean & F & Sig. \\
& Sum of & & Square & & \\
& Squares & & & & \\
\hline Method & 3.551 & 1.000 & 3.551 & 0.703 & 0.415 \\
\hline Error(Method) & 75.782 & 15.000 & 5.052 & & \\
\hline Lap & 949.297 & 4.000 & 237.32 & 262.466 & 0.000 \\
\hline Error(Lap) & & & 4 & & \\
\hline Method * Lap & 299.540 & 4.000 & 74.885 & 83.103 & 0.000 \\
\hline Error(Method*Lap) & 54.067 & 60.000 & 0.901 & & \\
\hline
\end{tabular}




\section{BIBLIOGRAPHY}

1. Ainslie PN, Reilly T, Westerterp KR. Estimating human energy expenditure: A review of techniques with particular reference to doubly labeled water. Sports Medicine 2003; 33 (9): 683688.

2. American College of Sports Medicine. (2006). ACSM's Guidelines for Exercise Testing and Prescription. 7th ed. Baltimore, MD: Lippincott Williams and Wilkins.

3. Andre D, et al. The development of the SWA armband, a revolutionary energy assessment device to assess physical activity and lifestyle, in White Papers Body Media www.bodymedia.com. 2006: Pittsburgh.

4. Baranowski T. Validity and reliability of self-report measures of physical activity: An information-processing perspective. Res Q Exerc Sport 1988; 59: 314-327.

5. Benito PJ, Neiva C, Gonzalez-Quijano PS, Cupeiro R, Morencos E, Peinado AB. Validation of the Sensewear armband in circuit resistance training with different loads. Eur $\mathrm{J}$ Appl Physiol 2011; Dec 06 Short Communication.

6. Berntsen S, et al. Validity of physical activity monitors in adults participating in freeliving activities. Br J Sports Med 2010; 44: 657-664

7. Bland JM, Altman DG. Measurement in medicine: the analysis of method comparison studies. The Statistician 1983; 32: 302-317

8. Bland JM, Altman DG. Measuring agreement in method comparison studies. Statistical Methods in Medical Research 1999; 135-160

9. Bouten $\mathrm{CV}$, et al. Assessment of energy expenditure for physical activity using triaxial accelerometer. Med Sci Sports Exerc 1994; 26(12):1516-1523.

10. Brage S, Brage N, Ekelund U, Luan J, Franks PW, Froberg K and Wareham NJ. Effect of combined movement and heart rate monitor placement on physical activity estimates during treadmill locomotion and free living. European Journal of Applied Physiology. 2006; 96: 517524. 
11. Brage S, Brage N, Franks PW, Ekelund U and Wareham NJ. Reliability and validity of the combined heart rate and movement sensor Actiheart. European Journal of Clinical Nutrition.2005; 59: 561-570.

12. Brown, D, et al. Analysis of gaseous exchange in open-circuit indirect calorimetry, Medical \& Biological Engineering \& Computing 1984; 22: 333-338

13. Campbell KL, et al. Field evaluation of energy expenditure in women using tritrac accelerometers. Med Sci Sports Exerc 2002; 34 (10): 1667-1674.

14. Cessay, SM, Prentice, AM, Day, KC, Murgatroyd, PR, Goldberg, GR, Scott, W. The use of heart rate monitoring in the estimation of energy expenditure: validation study using indirect calorimetry. British Journal of Nutrition 1989; 61; 175-186

15. Chen KY, Bassett DR. The technology of accelerometry-based activity monitors: current and future Med. Sci. Sports Exerc 2005; 37(11) Suppl: S490-S500

16. Christensen CC, et al. A critical evaluation of energy expenditure estimates based on individual $\mathrm{O}_{2}$ consumption/heart rate curves and average daily heart rate. Am J Clin Nutr 1983; 37: 468-472

17. Clausius R. Mechanical theorem applicable to heat. Philisophical Magazine 1870; 40; 265: $122-127$

18. Coward WA. The doubly-labeled-water $\left({ }^{2} \mathrm{H}_{2}{ }^{18} 0\right)$ method: principles and practice. Proceedings of the Nutrition Society 1988; 47: 209-218

19. Coward WA. Measurement of energy expenditure: the doubly-labeled-water method in clinical practice. Proceedings of the Nutrition Society 1991; 50: 227-237

20. Crawford K, et al. Validation of SWA Armband to assess EE of adolescents during various modes of activity. Med Sci Sports Exerc 2005; 37 (5) Supplement: S337.

21. Dauncey MJ, James WPT. Assessment of the heart-rate method for determining energy expenditure in man, using whole-body calorimeter. Br. J. Nutr 1979; 42: 1-13

22. Drenowatz C, Eisenmann JC. Validation of the sensewear armband at high intensity exercise. Eur J Appl Physiol 2011; 111: 883-887

23. Duffield R, Dawson B, Pinnington HC and Wong P. Accuracy and reliability of a Cosmed K4b2 portable gas analysis system. Journal of Science, Medicine and Sport 7(1): 11-22, 2004.

24. Ekelund U, et al. Physical activity assessed by activity monitor and doubly labeled water in children. Med. Sci. Sports Exerc 2001; 33(2): 275-281

25. Ekelund U, et al. Energy expenditure assessed by heart rate and doubly labeled water in young athletes. Med. Sci. Sports Exerc 2002; 34(8): 1360-1366 
26. Farringdon J, Wolf D. Does heart rate predict energy expenditure? Yes, but..... in White Papers Body Media www.bodymedia.com, 2006:Pittsburgh.

27. Farringdon J, Nashold S. Continuous body monitoring, Ambient Intelligence for Scientific Discovery 2005; 3345: 202-223

28. Ferrannini E. The theoretical base of indirect calorimetry: A Review. Metabolism 1988; 37(3):287-301.

29. Forbes-Ewan $\mathrm{CH}$, et al. Use of doubly labeled water technique in soilders training for jungle warefare. J. Appl. Physiol 1989; 67(1): 14-18

30. Freedson PS, Miller K. Objective monitoring of physical activity using motion sensors and Heart Rate. Res Q Exerc Sport 2000;71(2):21-29.

31. Fruin ML, Rankin JW. Validity of a multi-sensor armband in estimating rest and exercise EE. Med Sci Sports Exerc 2004; 36(6): 1063-1069.

32. Goodie JL, Larkin KT, Schauss S. Validation of the Polar heart rate monitor for assessing heart rate during physical and mental stress. Journal of Psychophysiology. 2000; 14(3): 159-164.

33. Haggarty P, McGraw BA. Non-restrictive methods for measuring energy expenditure. Proceedings of the Nutrition Society 1988; 47: 365-374

34. Haymes EM, Byrnes WC. Walking and running energy expenditure estimated by caltrac and indirect calorimetry. Med. Sci. Sports Exerc 1993; 25(12): 1365=1369

35. Hendelman D, Miller K, Bagget C, Debold E, Freedson P. Validity of accelerometry for the assessment of moderate intensity physical activity in the field. Med Sci Sports Exerc 2000; 32(9) Supplement: S442-S449.

36. Hiilloskorpi H, Fogelholm M, Laukkanen R, Pasanen M, Oja P, Manttari A, Natri A. Factors affecting the relation between heart rate and energy expenditure during exercise. International Journal of Sports Medicine 1999; 20:438-443.

37. Hiilloskorpi HK, et al. Use of heart rate to predict energy expenditure from low to high activity levels. Int J Sports Med 2003; 24: 332-336

38. Hoyt RW. Doubly labeled water measurement of human energy expenditure during strenuous exercise. J. Appl. Physiol 1991; 71(1): 16-22

39. Jakicic JM, et al. Evaluation of the sensewear pro armband to assess energy expenditure during exercise. Med. Sci. Sports Exerc 2003; 36 (5): 897-904

40. Johannsen DL, et al. Accuracy of armband monitors for measuring daily energy expenditure in healthy adults. Med. Sci. Sports Exerc; 42(11): 2134-2140 
41. Keytel LR, Goedecke JH, Noakes TD, Hiiloskorpi H, Laukkanen R, Van Der Merwe L, Lambert EV. Prediction of energy expenditure from heart rate monitoring during submaximal exercise. Journal of Sports Science 2005; 23(3): 289-297.

42. King GA, et al. Comparison of activity monitors to estimate energy cost of treadmill exercise. Med Sci Sports Exerc 2004; 36(7):1244-1251.

43. Koehler $\mathrm{K}$, et al. Assessing energy expenditure in male endurance athletes: validity of the sensewear armband. Med. Sci. Sports Exerc; 36(5): 897-904

44. Kumahara $\mathrm{H}$, et al. The use of uniaxial accelerometry for the assessment of physicalactivity-related energy expenditure: validation study against whole-body indirect calorimetry. $\mathrm{Br}$ J Nutr 2004; 91: 235-243

45. Lamonte, MJ., Ainsworth, BE., Quantifying energy expenditure and physical activity in the context of dose response, Medicine \& Science in Sports \& Exercise 2001; 33: S370-S378

46. Leeders N, et al. Evaluation of methods to assess physical activity in free-living conditions. Med. Sci. Sports Exerc 2001; 33(7): 1233-1240

47. Leger, LA., Lambert, J., A maximal multistage 20-m shuttle run test to predict $\mathrm{VO}_{2}$ max, European Journal Applied Physiology 1982; 49:1-12

48. Leonard WR., Measuring human energy expenditure and metabolic function: basic principles and methods, Journal of Anthropological Sciences 2010; 88: 221-230

49. Levine, JA., Measurement of energy expenditure, Public Health Nutrition 2005; 8(7A): 1123-1132

50. Li, R, Deurenberg, P, Hautvast, JG, A critical evaluation of heart rate monitoring to assess energy expenditure in individuals. American Journal of Clinical Nutrition 1993; 58; 602607

51. Linden CB, et al. Benefits of the SWA Armband over other physical activity and Energy Expenditure measurement techniques, in White Papers Body Media www.bodymedia.com, 2001:Pittsburgh.

52. Linden CB, et al. Accuracy and Reliability of the SWA Armband as an energy expenditure assessment device, in White Papers Body Media www.bodymedia.com, 2006:Pittsburgh.

53. Livingstone MBE, et al. Simultaneous measurement of free-living energy expenditure by the doubly labeled water method and heart rate monitoring. Am J Clin Nutr 1990; 52: 59-65

54. McLaughlin JE, King GA, Howley ET,Jr. DR, Ainsworth BE. Validation of the COSMED K4 b² Portable Metabolic System. International Journal of Sports Med 2001; 22:280284. 
55. McArdle, Katch, Katch. (2011) Exercise Physiology: Energy, Nutrition, \& Performance. 6th ed. Baltimore, MD: Lippincott Williams and Wilkins.

56. Mealey AD, et al. Validation of the SenseWear Pro Armband to estimate energy expenditure during a simulation of daily activity. Abstract Presented at the Annual Meeting of the American College of Sports Medicine 2007, New Orleans, LA. USA.

57. Meijer GA, et al. Assessment of energy expenditure by recording heart rate and body acceleration. Med. Sci. Sports Exerc 1989; 21(3): 343-347

58. Montoye HJ, et al. Estimation of energy expenditure by a portable accelerometer. Med. Sci. Sports Exerc 1983; 15(5): 403-407

59. Nichols JF, et al. Validity, reliability, and calibration of the tritrac accelerometer as a measure of physical activity. Med. Sci. Sports Exerc 1999; 31(6): 908-912

60. Ostojic SM, Mazic S, Dikic N. Profiling in basketball: physical and physiological characteristics of elite players. J of Strength and Cond Res 2006; 20(4): 740-744

61. Passmore, R, Durnin, J. Energy expenditure and calorie requirements. Human Energy Expenditure 1955; 35: 801-840

62. Rennie, KL., Wareham, NJ., The validation of physical activity instruments for measuring energy expenditure: problems and pitfalls, Public Health Nutrition; 1(4): 265-271

63. Roberts, SB, Heyman, MB, Evans, WJ, Fus, P, Tsay, R, Young, VR. Dietary energy requirements of young adult men, determined by using the doubly labeled water method. American Journal of Clinical Nutrition 1991; 54; 499-505.

64. Robertson, RJ, (2004) Perceived exertion for practitioners, rating effort with the OMNI picture system. Champaign, IL: Human Kinetics.

65. Rowlands, AV, et al., Validation of the RT3 triaxial accelerometer for the assessment of physical activity. Med. Sci. Sports Exerc 2004; 36(3): 518-524

66. Rozzi, SL, et al., Knee joint laxity and neuromuscular characteristics of male and female soccer and basketball players. Am J Sports Medicine 1999; 27 (3): 312-319

67. Schoeller, DA, Measurement of energy expenditure in free-living humans by using doubly labeled water. J Nutr 1988; 118: 1278-1289.

68. Schoeller DA, Webb P. Five-day comparison of doubly labeled water method with respiratory gas exchange. Am J Clin Nutr 1984; 40:153-158.

69. Schoeller DA, et al. Energy Expenditure by doubly labeled water: validation in humans and proposed calculation. American Physiological Society 1986; 19: R823-R830 
70. Schoeller DA, et al. Recent Advances from application of doubly labeled water to measurement of human energy expenditure. J Nutr 1999; 129: 1765-1768

71. Schulz LO. Energy expenditure of elite female runners measured by respiratory chamber and doubly labeled water. J. Appl. Physiol 1992; 72(1): 23-28

72. Schulz, LO., Schoeller, DA., A compilation of total daily energy expenditure and body weights in adults, American Journal of Clinical Nutrition 1994; 60: 676-681

73. Seale JL, Conway JM, Canary JJ. Seven-day validation of doubly labeled water method using indirect room calorimetry. J Appl Physiol 1993; 74(1): 402-409.

74. Sigmon, C, (2003) 52-Week basketball training, a proven plan for strength, power, speed, agility and performance. Champaign, IL: Human Kinetics.

75. Simonson, DC., DeFronzo, RA., Indirect Calorimetry: methodological and interpretive problems, The American Physiological Society 1990; 193: 1849

76. Soric, M., Mikulic, P., Misigoj-Durakovic, M., Ruzic, L., Markovic, G., Validation of the Sensewear armband during recreational in-line skating, Eur J Appl Physiol 2012; 112: 11831188

77. Spurr GB, et al. Energy expenditure from minute-by-minute heart-rate recording: comparison with indirect calorimetry. Am J Clin Nutr 1988; 48: 552-559

78. St-Onge M, Mignault D, Allison DB and Rabasa-Lhoret R. Evaluation of a portable device to measure daily energy expenditure in free-living adults. The American Journal of Clinical Nutrition. 2007; 85: 742-749. 5.

79. Strath SJ, Bassett DR, Swartz AM, Thompson DL. Simultaneous heart rate-motion sensor technique to estimate energy expenditure. Med Sci Sports Exerc 2001; 33(12): 21182123.

80. Strath SJ, Bassett DR, Thompson DL, Swartz AM. Validity of the simultaneous heart rate-motion sensor technique for measuring energy expenditure. Med Sci Sports Exerc 2002;34 (5):888-894.

81. Stuempfle KJ, et al., Change in serum sodium concentration during a cold weather ultraindurance race. Cli J Sports Med 2003; 13(3):171-175.

82. Swartz AM, et al., Estimation of energy expenditure using CSA accelerometers at hip and wrist sites. Med. Sci. Sports Exerc 2000; 32(9) Supplement: S450-S456

83. Teller A. A platform for wearable physiological computing, in White Papers Body Media www.bodymedia.com, 2004:Pittsburgh.

84. Teller A. Ambient and wearable computing, in White Papers Body Media www.bodymedia.com, 2004:Pittsburgh. 
85. Terbizan DJ, Dolezal BA, Albano C. Validity of seven commercially available heart rate monitors. Measurement in Physical Education and Exercise Science. 2002; 6(4): 243-247.

86. Westerterp, KR., Impacts of vigorous activity on daily energy expenditure, Preceedings of the Nutrition Society 2003; 62: 645-650

87. Westerterp KR, et al. Comparison of doubly labeled water with respirometry at low- and high-activity levels. J. Appl. Physiol 1988; 65(1): 53-56

88. Westerterp KR, et al. Use of the doubly labeled water technique in humans during heavy sustained exercise. J. Appl. Physiol 1986; 61(6): 2162-2167

89. Wicks JR, et al. HR index-a simple method for prediction of oxygen uptake. Med. Sci. Sports Exerc 2011; 43(10): 2005-2012

90. Woolstenhulme MT, Bailey BK, Allsen PE. Vertical jump, anaerobic power, and shooting accuracy are not altered 6 hours after strength training in collegiate women's basketball players. J Strength Cond Res 2004 Aug; 18 (3): 422-5

91. Wolff, HS., Modern Techniques for measuring energy expenditure. Energy expenditure in man 1956; 15: 77-80

92. Zhang, K, et al. Improving energy expenditure estimation for physical activity, Med. Sci. Sports Exerc; 36(5): 883-889

93. K4b2 Installation and start-up information. Retrieved May 27, 2011, from http://cosmed.it

94. Body composition and Tanita, Science and Innovation. Retrieved November 14, 2011, from http://www.tanita.com/en/science-innovation/5 